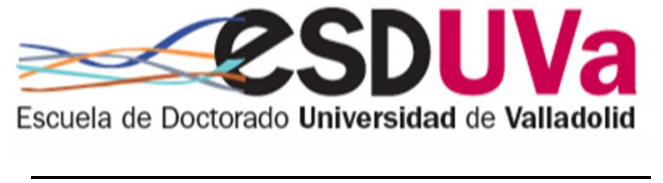

Universidad deValladolid

TESIS DOCTORAL

\title{
CLIINICA ANSIOSO-DEPRESIVA Y CALIDAD DE VIDA EN LA ENFERMEDAD DE PARKINSON
}

Presentada por Fany Chuquilín Arista para optar al grado de

Doctora por la Universidad de Valladolid

\author{
Dirigida por: \\ Dr. Manuel Menéndez González
}

Tutora:

Dra. María Jesús Coma Del Corral

Dra. María Natividad García Atares

Valladolid, 2020 
A la memoria de mi padre.

A mi madre y a mi hermano 


\section{AGRADECIMIENTOS}

Al Dr. Manuel Menéndez González, por su apoyo y orientación en la realización de este proyecto.

A mis tutoras de tesis, la Dra. María Jesús Coma del Corral, quien me apoyó incondicionalmente al iniciar este proyecto; y la Dra. María Natividad García Atares, que continuó con la tutoría hasta su finalización.

A la Unidad de Consultoría Estadística de la Universidad de Oviedo por su apoyo en el análisis de datos.

A todos los miembros de la Asociación Parkinson Asturias porque sin su participación hubiera sido imposible la realización de este proyecto.

A todos los que permitieron con su colaboración la realización de este proyecto. 


\section{ÍNDICE}

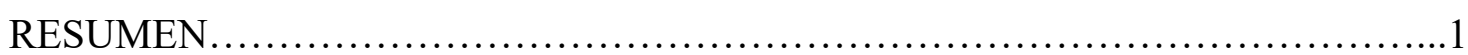

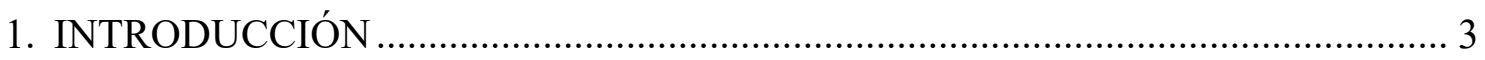

1.1 Datos generales.......................................................4

1.2 Enfermedad de Parkinson en España.................................................5

1.3 Asociaciones de enfermedad de Parkinson.....................................5

1.4 Etiología y Fisiopatología de la enfermedad de Parkinson........................6

1.5 Clínica y Diagnóstico de la Enfermedad de Parkinson............................7

1.6 Parkinson y emociones............................................. 10

1.6.1 Depresión.........................................................11

1.6.2 Ansiedad.......................................................... 12

1.7 Calidad de vida............................................................. 14

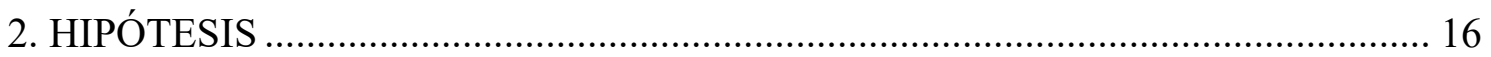

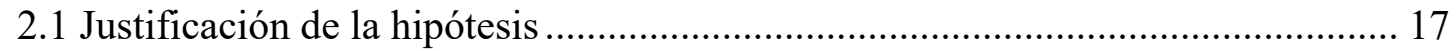

2.2 Hipótesis de trabajo................................................18

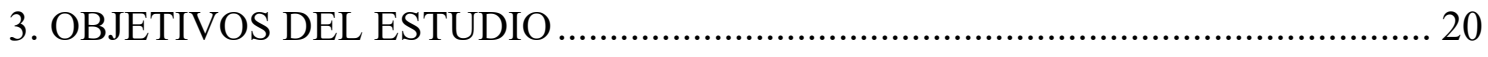

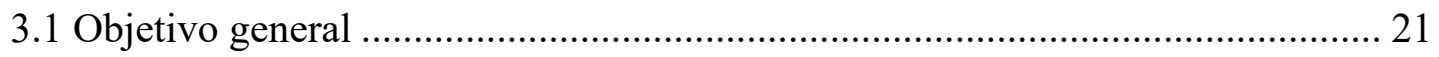

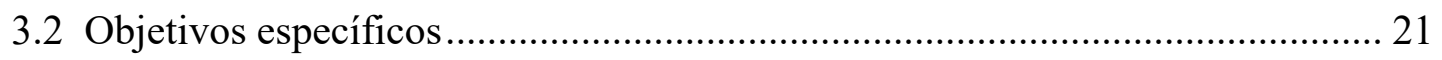

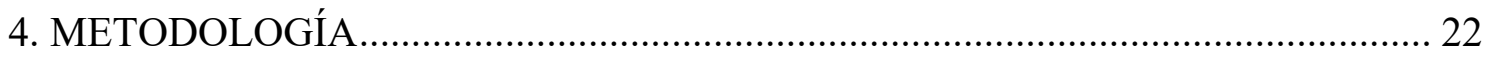

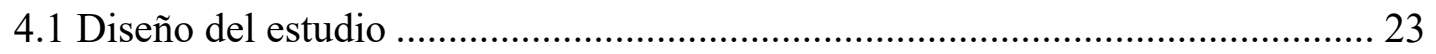

4.2 Población de estudio...................................................23

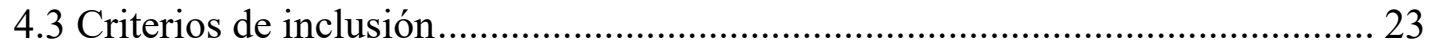

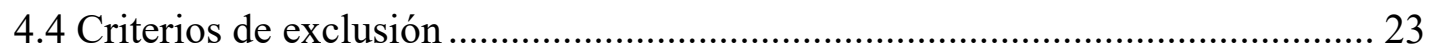

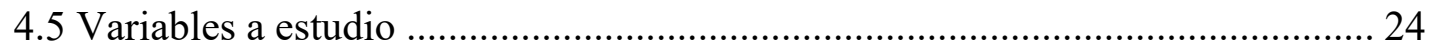

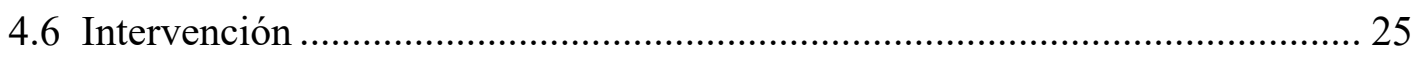

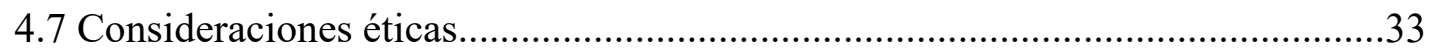

4.8 Análisis stadístico......................................................

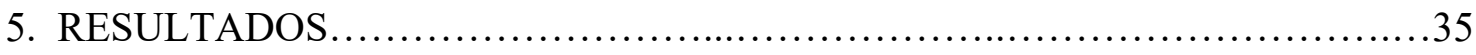


5.1 Descripción de la muestra..................................................

5.2 Prevalencia de depresión y su relación con las variables....................46

5.3 Prevalencia de ansiedad y su relación con las variables....................56

5.4 Calidad de vida y su relación las variables..................................................68

5.4.1 Calidad de vida: Movilidad.....................................69

5.4.2 Calidad de vida: Actividades de la vida diaria.......................75

5.4.3 Calidad de vida: Bienestar emocional...........................80

5.4.4 Calidad de vida: Estigmatización...............................85

5.4.5 Calidad de vida: Apoyo social......................................90

5.4.6 Calidad de vida: Estado cognitivo...................................95

5.4.7 Calidad de vida: Comunicación................................100

5.4.8 Calidad de vida: Dolor......................................... 105

5.4.9 Calidad de vida: PDSI.....................................110

5.4.10 Calidad de Vida: Regresión lineal múltiple......................115

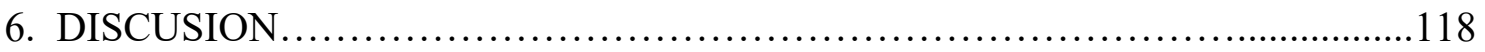

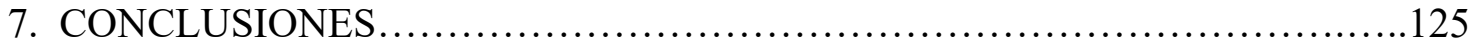

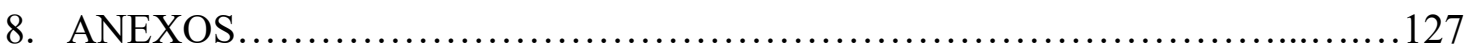

Anexo 1: Estudios de prevalencia de la enfermedad de Parkinson en España....................................................... 128

Anexo 2: Clasificación por Estadíos de Hoehn y Yahr ....................................... 129

Anexo 3: Mini-Examen Cognoscitivo (MEC-30).......................... 130

Anexo 4: Cuestionario de Calidad de Vida en Enfermedad de Parkinson

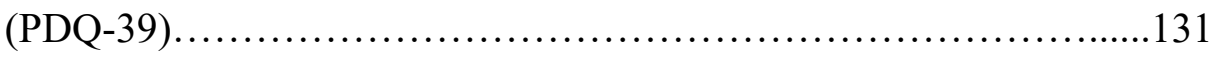

Anexo 5: Cuestionario de STAI....................................... 133

Anexo 6: Baremos de puntuaciones del STAI..............................135

Anexo 7: Cuestionario de Beck..........................................................136

Anexo 8: Autorización del Comité de Ética de la Investigación del

Principado de Asturias..............................................138

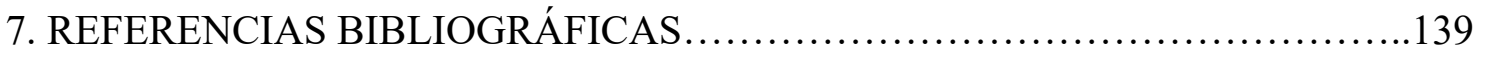




\section{ÍNDICE DE TABLAS}

Tabla 1: Criterios Clínicos de Diagnóstico de la Enfermedad de Parkinson (EP) del Banco de Cerebros de la Sociedad de Enfermedad de Parkinson del Reino Unido.............................................9

Tabla 2: Variables de estudio...............................................26

Tabla 3: Características generales de la población................................39

Tabla 4: Presencia de comorbilidades.......................................41

Tabla 5: Presencia de otros trastornos neuropsiquiátricos........................41

Tabla 6: Distribución de tratamiento antiparkinsoniano........................42

Tabla 7: Tipos de tratamiento antiparkinsoniano.............................43

Tabla 8: Otros tratamientos..................................................44

Tabla 9: Calidad de vida.................................................45

Tabla 10: Prevalencia de depresión..........................................46

Tabla 11: Tratamiento antidepresivo y ansiolítico en BDI-II alterado..................47

Tabla 12: Relación de la depresión y variables sociodemográficas y estado Cognitivo...................................................48

Tabla 13: Relación de la depresión y características de la Enfermedad de Parkinson..........................................................49

Tabla 14: Relación de la depresión y tratamientos antiparkinsonianos................50

Tabla 15: Relación de la depresión y otras enfermedades médicas..................51

Tabla 16: Relación entre depresión y antecedente de patología de Salud Mental.........51

Tabla 17: Relación de la depresión y la calidad de vida según el PDQ-39.............53

Tabla 18: Relación del PDSI y depresión......................................54

Tabla 19: Análisis de regresión logística múltiple de la depresión y otras 
variables

Tabla 20: Puntuación directa y centil del STAI según sexo.........................56

Tabla 21: Estratificación de los niveles de ansiedad.............................58

Tabla 22: Tratamiento ansiolítico y antidepresivo en pacientes con ansiedad..........59

Tabla 23: Coexistencia de depresión y ansiedad según sexo........................59

Tabla 24: Relación de la ansiedad y las diversas variables.........................60

Tabla 25: Relación de la ansiedad y características de la Enfermedad de Parkinson..............................................................61

Tabla 26: Relación de la ansiedad y tratamientos antiparkinsonianos.................62

Tabla 27: Relación de la ansiedad y otras enfermedades médicas....................63

Tabla 28: Relación de la ansiedad y antecedente de patología de Salud Mental.........64

Tabla 29: Relación de la ansiedad y la calidad de vida según el PDQ-39..............65

Tabla 30: Relación del PDSI y ansiedad........................................66

Tabla 31: Análisis de regresión logística múltiple de la ansiedad y otras variables.....67

Tabla 32: Relación del PDQ-39: Movilidad y características generales................70

Tabla 33: Relación del PDQ-39: Movilidad y características de la Enfermedad de Parkinson........................................................... 71

Tabla 34: Relación del PDQ-39: Movilidad y tratamientos antiparkinsonianos.........72

Tabla 35: Relación del PDQ-39: Movilidad y otras enfermedades médicas..............73

Tabla 36: Relación del PDQ-39: Movilidad y antecedente de patología de Salud Mental.

Tabla 37: Relación del PDQ-39: Movilidad y tratamiento antidepresivo y 
ansiolítico

Tabla 38: Relación del PDQ-39: Movilidad y presencia de depresión o ansiedad .74

Tabla 39: Relación del PDQ-39: Actividades de la vida diaria y características generales. .75

Tabla 40: Relación del PDQ-39: Actividades de la vida diaria y características de la Enfermedad de Parkinson. .76

Tabla 41: Relación del PDQ-39: Actividades de la vida diaria y tratamientos antiparkinsonianos. .77

Tabla 42: Relación del PDQ-39: Actividades de la vida diaria y otras enfermedades médicas. . .78

Tabla 43: Relación del PDQ-39: Actividades de la vida diaria y antecedente de patología de Salud Mental.

Tabla 44: Relación del PDQ-39: Actividades de la vida diaria y tratamiento antidepresivo y ansiolítico.

Tabla 45: Relación del PDQ-39: Actividades de la vida diaria y presencia de depresión o ansiedad.

Tabla 46: Relación del PDQ-39: Bienestar emocional y características generales .80

Tabla 47: Relación del PDQ-39: Bienestar emocional y características de la Enfermedad de Parkinson. .81

Tabla 48: Relación del PDQ-39: Bienestar emocional y tratamientos antiparkinsonianos....

Tabla 49: Relación del PDQ-39: Bienestar emocional y otras enfermedades médicas

Tabla 50: Relación del PDQ-39: Bienestar emocional y antecedente de patología de Salud Mental. 
Tabla 51: Relación del PDQ-39: Bienestar emocional y tratamiento antidepresivo y ansiolítico

Tabla 52: Relación del PDQ-39: Bienestar emocional y presencia de depresión o ansiedad.

Tabla 53: Relación del PDQ-39: Estigmatización y características generales .84

Tabla 54: Relación del PDQ-39: Estigmatización y características de la Enfermedad de Parkinson .86

Tabla 55: Relación del PDQ-39: Estigmatización y tratamiento antiparkinsonianos

Tabla 56: Relación del PDQ-39: Estigmatización y otras enfermedades médicas .88

Tabla 57: Relación del PDQ-39: Estigmatización y antecedente de patología de Salud Mental .88

Tabla 58: Relación del PDQ-39: Estigmatización y tratamiento antidepresivo y ansiolítico

Tabla 59: Relación del PDQ-39: Estigmatización y presencia de depresión o ansiedad. 89

Tabla 60: Relación del PDQ-39: Apoyo social y características generales .90

Tabla 61: Relación del PDQ-39: Apoyo social y características de la Enfermedad de Parkinson 91

Tabla 62: Relación del PDQ-39: Apoyo social y tratamientos antiparkinsonianos......92

Tabla 63: Relación del PDQ-39: Apoyo social y otras enfermedades médicas. .93

Tabla 64: Relación del PDQ-39: Apoyo social y antecedente de patología de Salud Mental. .93

Tabla 65: Relación del PDQ-39: Apoyo social y tratamiento antidepresivo y ansiolítico 
Tabla 66: Relación del PDQ-39: Apoyo social y presencia de depresión o ansiedad

Tabla 67: Relación del PDQ-39: Estado cognitivo y características generales. .95

Tabla 68: Relación del PDQ-39: Estado cognitivo y características de la Enfermedad de Parkinson. .96

Tabla 69: Relación del PDQ-39: Estado cognitivo y tratamientos antiparkinsonianos. .97

Tabla 70: Relación del PDQ-39: Estado cognitivo y otras enfermedades médicas......98

Tabla 71: Relación del PDQ-39: Estado cognitivo y antecedente de patología de Salud Mental. .98

Tabla 72: Relación del PDQ-39: Estado cognitivo y tratamiento antidepresivo y ansiolítico.

Tabla 73: Relación del PDQ-39: Estado cognitivo y presencia de depresión o ansiedad.

Tabla 74: Relación del PDQ-39: Comunicación y características generales .100

Tabla 75: Relación del PDQ-39: Comunicación y características de la Enfermedad de Parkinson.

Tabla 76: Relación del PDQ-39: Comunicación y tratamientos antiparkinsonianos. 102

Tabla 77: Relación del PDQ-39: Comunicación y otras enfermedades médicas 103

Tabla 78: Relación del PDQ-39: Comunicación y antecedente de patología de Salud Mental.

Tabla 79: Relación del PDQ-39: Comunicación y tratamiento antidepresivo y ansiolítico 104

Tabla 80: Relación del PDQ-39: Comunicación y presencia de depresión o ansiedad 
Tabla 81: Relación del PDQ-39: Dolor y características generales .105

Tabla 82: Relación del PDQ-39: Dolor y características de la Enfermedad de Parkinson 106

Tabla 83: Relación del PDQ-39: Dolor y tratamientos antiparkinsonianos... 107

Tabla 84: Relación del PDQ-39: Dolor y otras enfermedades médicas. 108

Tabla 85: Relación del PDQ-39: Dolor y antecedente de patología de Salud Mental. 108

Tabla 86: Relación del PDQ-39: Dolor y tratamiento antidepresivo y ansiolítico

Tabla 87: Relación del PDQ-39: Dolor y presencia de depresión o ansiedad..... 109

Tabla 88: Relación del PDQ-39: PDSI y características generales

Tabla 89: Relación del PDQ-39: PDSI y características de la Enfermedad de Parkinson

Tabla 90: Relación del PDQ-39: PDSI y tratamientos antiparkinsonianos.... 112

Tabla 91: Relación del PDQ-39: PDSI y otras enfermedades médicas

Tabla 92: Relación del PDQ-39: PDSI y antecedente de patología de Salud Mental

Tabla 93: Relación del PDQ-39: PDSI y tratamiento antidepresivo y ansiolítico .114

Tabla 94: Relación del PDQ-39: PDSI y presencia de depresión o ansiedad.... 115

Tabla 95: Análisis de regresión lineal de la calidad de vida y otras variables .117 


\section{ÍNDICE DE FIGURAS}

Figura 1: Pacientes de la Asociación de Parkinson Asturias según sedes estudiadas. .36

Figura 2: Distribución de la gravedad de la Enfermedad de Parkinson

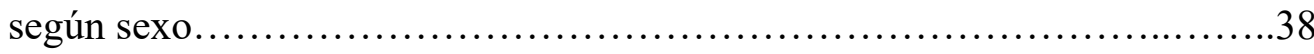

Figura 3: Niveles de depresión según sexo.................................46

Figura 4: Presencia de Ansiedad Estado elevado según sexo.........................57

Figura 5: Presencia de Ansiedad Rasgo según sexo..................................57

Figura 6: Distribución de la ansiedad por intensidad............................58

Figura 7: Puntuaciones medias de las dimensiones del PDQ-39 y el PDSI......................68 


\author{
ABREVIATURAS
}

BDI-II Inventario de Depresión de Beck-II

CVRS Calidad de vida relacionada con la salud

DS Desviación estándar.

EP Enfermedad de Parkinson.

F.E.P. $\quad$ Federación Española de Parkinson

FOESSA Fomento de Estudios Sociales y Sociología Aplicada.

INE Instituto Nacional de Estadística

ISCED The International Standard Classification of Education

LRRK2 Leucine-rich repeat kinase 2

MEC-30 Mini-Examen Cognoscitivo

OMS Organización Mundial de la Salud

PDQ-8 Parkinson's Disease Questionnaire-8

PDQ-39 Cuestionario de Calidad de Vida en Enfermedad de Parkinson

PDSI Parkinson's disease summary index

SEN Sociedad Española de Neurología

STAI Cuestionario de Ansiedad Estado-Rasgo

STAI-E Cuestionario de Ansiedad Estado.

STAI-R Cuestionario de Ansiedad Rasgo. 


\section{RESUMEN}

Introducción: La enfermedad de Parkinson (EP) es una enfermedad neurodegenerativa que se caracteriza por presentar tanto sintomatología motora como no motora, destacando de este último grupo los síntomas neuropsiquiátricos. La mayor parte de los estudios realizados sobre esta enfermedad en las décadas precedentes, se centraban en los síntomas motores obviando los síntomas psicológicos asociados.

Los pacientes con enfermedad de Parkinson tienen un aumento en la prevalencia de patología ansioso-depresiva, aunque los valores varían según diversos estudios. Además, al considerar la enfermedad de forma global se puede valorar de forma adecuada la repercusión en la calidad de vida del paciente.

Objetivos: Establecer la prevalencia de la clínica ansioso-depresiva en los pacientes con enfermedad de Parkinson inscritos en la "Asociación Parkinson Asturias" a Marzo 2017 y su relación con diversos factores sociodemográficos y clínicos. Determinar la calidad de vida de los pacientes con enfermedad de Parkinson y su relación con la clínica ansiosodepresiva.

Metodología: Se realizó un estudio observacional, descriptivo y transversal realizado con 95 pacientes diagnosticados de EP en distintos estadíos de la enfermedad. La ansiedad y la depresión fueron valoradas mediante el Cuestionario de Ansiedad Estado-Rasgo (STAI) y el Cuestionario de Depresión de Beck (BDI-II) respectivamente y la calidad de vida por el Cuestionario de Calidad de Vida en Enfermedad de Parkinson (PDQ-39). Se excluyeron a los pacientes que tenían un deterioro cognitivo severo (MEC-30<12), que no pudieran leer o escribir o que estuvieran cursando con una enfermedad en fase terminal.

Resultados: Se encontró una prevalencia de depresión del 32.63\% y de ansiedad (STAI/R) del 68.42\%. La coexistencia de depresión y ansiedad fue del $31.58 \%$ de la población estudiada. Sorprendentemente, no se encontró relación de estos síntomas con las variables sociodemográficas $\mathrm{ni}$ con otras comorbilidades o tratamientos 
antiparkinsonianos recibidos. Se encontró asociación entre el tiempo de evolución de la EP y la depresión, la disminución leve del estado cognitivo y la ansiedad; así como entre la presencia de ansiedad, depresión y un mayor estadío de la EP (estadíos de Hoehn and Yahr) con la calidad de vida relacionada con la salud de los pacientes.

Todas las dimensiones de la calidad de vida, excepto las dimensiones de soporte social y malestar corporal, se relacionaron con la presencia de depresión. La ansiedad tuvo un impacto negativo en las dimensiones de estado emocional y malestar corporal.

Conclusiones: Un tercio de los pacientes con EP presentan depresión y más de dos tercios presentan ansiedad. La clínica ansioso-depresiva tiene un gran impacto en la calidad de vida relacionada con la salud de los pacientes. La sintomatología depresiva tiene un impacto negativo en la mayoría de las dimensiones de la calidad de vida. 


\section{INTRODUCCIÓN}




\section{INTRODUCCIÓN}

\subsection{Datos generales}

La enfermedad de Parkinson (EP) es la segunda enfermedad neurodegenerativa más frecuente a nivel mundial, después de la enfermedad de Alzheimer ${ }^{(1)}$. Se estima que la EP afecta al 1-2\% de los mayores de 65 años, aumentando su incidencia y prevalencia a mayor edad. La edad media de inicio es de 60 años, siendo el 10\% de los afectados menores de 45 años. Afecta a todas las razas y es discretamente más frecuente en varones (1.5 veces) que en las mujeres ${ }^{(2)}$.

Las primeras referencias que se tienen de esta enfermedad se remontan al siglo I a.C, en los tratados ayurvédicos indios y en textos chinos donde mencionan síntomas como la hipersalivación, el temblor, la rigidez generalizada o la somnolencia, sintomatología propia de la enfermedad de Parkinson. La referencia clásica más conocida sería la de Galeno de Pérgamo (129-199) quien describió el temblor y estableció las diferencias entre el temblor de reposo y el intencional ${ }^{(3)}$.

Propiamente, la enfermedad fue descrita por primera vez en 1817 por el médico inglés James Parkinson en su publicación “An Essay on the shaking palsy” donde, luego de tres décadas de experiencia clínica, describe las alteraciones del movimiento de esta enfermedad a la que denominó "parálisis agitante" (4). La descripción inicial es muy completa, pero no menciona la rigidez ni los trastornos cognoscitivos.

Años más tarde Charcot y Vulpain publican “De la paralysie agitante” (1861), donde describen la rigidez y la afectación de las facultades psíquicas. Además, Charcot fue el primero en sugerir el nombre de enfermedad de Parkinson a esta enfermedad ${ }^{(5)}$. Posteriormente se han publicado decenas de estudios sobre la historia natural de la enfermedad, con los cuales se ha llegado a conocer con mucho más detalle a esta enfermedad y distinguirla de otros trastornos del movimiento. 


\subsection{Enfermedad de Parkinson en España}

En España son muchos los estudios de prevalencia realizados. Los resultados de prevalencia obtenidos varían entre 150 y $1500 / 10^{5}$ habitantes principalmente debido a las diferencias metodológicas entre los mismos (Ver anexo 1) ${ }^{(6)}$. Según datos publicados en la "Guía Oficial de Práctica Clínica en la Enfermedad de Parkinson" de la Sociedad Española de Neurología (SEN), alrededor de 150.000 personas en España padecen la enfermedad de Parkinson, lo que supone el $0.3 \%$ de la población en general. La prevalencia aumenta al $2 \%$ en los mayores de 60 años y es superior al $4 \%$ de los mayores de 80 , con una incidencia de entre 8 y 18 casos nuevos casos por cada 100000 habitantes. Según esta misma guía, hasta el $24 \%$ de los pacientes han recibido un diagnóstico erróneo de Parkinson ${ }^{(7)}$.

Las proyecciones de prevalencia para el futuro indican una prevalencia en aumento, dado que la esperanza de vida es cada vez mayor. Actualmente, y según datos del Instituto Nacional de Estadística (INE), de los 46449565 habitantes de España al 01/01/2016, 11099849 tenían 60 años o más lo que correspondería al $23.89 \%$ de la población ${ }^{(8)}$. Por otro lado, Asturias es una de las comunidades con población más envejecida de España, por lo que es esperable el aumento de casos de enfermedad de Parkinson. Al 01/01/2015, tenía una población de 1049 875, de los cuales el 30.91\% (324 523) tenía 60 años o más ${ }^{(9)}$.

\subsection{Asociaciones de enfermedad de Parkinson}

Ante el impacto que ha tenido la enfermedad de Parkinson en la sociedad, se creó en Barcelona el año 1985, la primera asociación de Parkinson, con el objetivo de mejorar la calidad de vida de las personas afectadas y de sus familias. Sin embargo, no es hasta 1996 en que se constituye la Federación Española de Parkinson (F.E.P.) que actualmente cuenta con 45 asociaciones a nivel nacional y más de 12000 asociados ${ }^{(10)}$ organizados en asociaciones regionales. En Asturias la asociación toma el nombre de "Asociación Parkinson Asturias”. Fue creada en Octubre de 1997, pertenece a la F.E.P. y cuenta con 5 delegaciones ubicadas en Oviedo, Avilés, Langreo, Mieres y Jarrio ${ }^{(11)}$. 
La asociación brinda diversos servicios y terapias a sus socios: terapia ocupacional, musicoterapia, logopedia, fisioterapia, entre otros. Además, permite relacionarse con personas afectadas de los mismos problemas, creando grupos de soporte muy importantes para la vida del paciente y de sus familias.

Recientemente la "Asociación Parkinson Asturias" con el apoyo de la Fundación EDP, ha creado la escuela de Parkinson. Ésta es una plataforma web donde se realizan cursos y vídeo talleres gratuitos sobre el Parkinson y diversas terapias. Las personas que lo deseen pueden realizar una búsqueda de la información relativa al tema que le interesa y acceder a ella en cualquier momento, desde cualquier dispositivo y a su propio ritmo.

\subsection{Etiología y Fisiopatología de la enfermedad de Parkinson}

La etiología de la enfermedad de Parkinson es multifactorial, apareciendo la mayor parte de forma esporádica (85-90\%), siendo su causa desconocida. El otro 10-15\% tiene un origen familiar, identificándose múltiples mutaciones específicas y asociaciones génicas como la a-sinucleína y el LRRK2 (leucine-rich repeat kinase 2: PARK-8) cuya mutación causa una EP autosómica dominante con penetración del 70-100\% e inicio a partir de los 45 años ${ }^{(2)}$. También se han encontrado algunas mutaciones génicas en casos esporádicos ${ }^{(12)}$. Se cree que para que aparezca la enfermedad debería existir una predisposición genética y una exposición a un factor ambiental tóxico que induzca interacciones de ADN epigenéticas o somáticas ${ }^{(1,13)}$.

Existen diversas hipótesis patogénicas, todas las cuales concluyen que existiría una disfunción y muerte celular de las neuronas dopaminérgicas del locus niger. Como se sabe, la vía dopaminérgica nigroestriada inhibe a las neuronas estriatales que poseen receptores dopaminérgicos D2 y que conectan con el pálido interno, el núcleo eferente de los ganglios basales, después de la conexión con el núcleo subtalámico. Por este motivo, debido a la alteración de las neuronas dopaminérgicas del locus niger en la EP, la tasa de dopamina estriatal está disminuida, provocando una actividad excesiva subtalámica y palidal. Esta hiperactividad conlleva una menor activación tálamo-cortical y una reducción en los movimientos tanto espontáneos como automáticos. La EP se asocia también a hiperactividad de las interneuronas colinérgicas del estriado, lo que explicaría 
la mejoría que se observa en los síntomas cardinales de la enfermedad con la administración de fármacos anticolinérgicos.

Además, existe degeneración de otros sistemas neuronales en el tronco (núcleo dorsal del vago, locus coeruleus, núcleos del rafe), algunos de proyección ascendente (noradrenérgicos, serotoninérgicos y colinérgicos), y lesiones en la amígdala, el hipotálamo, la corteza cerebral y el sistema autonómico periférico que pueden estar implicados en la aparición de estados depresivos, deterioro cognitivo, alteraciones del sueño, y en la disfunción vegetativa, frecuentes en muchos pacientes ${ }^{(14)}$.

\subsection{Clínica y diagnóstico de la enfermedad de Parkinson}

Las manifestaciones de la enfermedad aparecen cuando la pérdida neuronal en la parte compacta de la sustancia negra es del $60-70 \%$ y la pérdida de dopamina en el estriado alcanza el $80 \%$. Este proceso es lento y tendría al menos varios años de duración. Este lapso de tiempo previo a que la enfermedad sea evidente se denomina periodo premotor. Durante el periodo premotor se pueden observar algunas manifestaciones clínicas como la depresión, el dolor, la pérdida de olfato, el trastorno del sueño REM, una ligera pérdida de agilidad, la alteración de la escritura, entre otros ${ }^{(15)}$.

Las manifestaciones clínicas cardinales de la EP son la bradicinesia, el temblor de reposo, la rigidez y la inestabilidad postural ${ }^{(16)}$. Su aparición suele ser insidiosa, de predominio asimétrico y en relación con la pérdida de dopamina del nigoestriado. En el 70-90\% de los casos, el síntoma de inicio es el temblor de reposo asimétrico que afecta a la mano. Aunque en los ancianos también es muy frecuente la bradicinesia ${ }^{(2)}$.

En estadios avanzados, el paciente camina con el tronco flexionado, arrastrando los pies, con pasos cortos y ausencia de balanceo de los brazos. En ocasiones, el ritmo de la marcha aumenta poco a poco (marcha festinante). También pueden existir bloqueos de la marcha con dificultad para iniciar o continuar un ritmo de pasos normal ${ }^{(14,17)}$.

Además de los síntomas motores, existen síntomas no motores que pueden resultar muchas veces molestos como los síntomas de disfunción vegetativa (estreñimiento, a veces grave, hiperhidrosis, sofocaciones y nicturia, polaquiuria, urgencia e 
incontinencia), la sialorrea, el dolor, las parestesias y las alteraciones del sueño. Por otro lado, un amplio porcentaje presentará deterioro cognitivo, la cual se asocia con frecuencia en estos pacientes a alucinaciones visuales y somnolencia diurna excesiva ${ }^{(14,18,19)}$.

El diagnóstico de la EP en vida es un diagnóstico de sospecha clínica. El diagnóstico “definitivo" precisa de la confirmación de los hallazgos neuropatológicos característicos, es decir, pérdida neuronal a nivel de la sustancia negra y la presencia de cuerpos y neuritas de Lewy, y solo puede hacerse una vez que el sujeto ha fallecido.

Existen diversos criterios para diagnosticar la enfermedad de Parkinson. Se basan en la presencia de 2 o 3 síntomas motores clásicos (bradicinesia, temblor, rigidez o inestabilidad), la exclusión de síntomas o signos atípicos, la respuesta a levodopa y la falta de exposición a fármacos ${ }^{(19-21)}$. La tabla 1 muestra los criterios diagnósticos del Banco de Cerebros de la Sociedad de Enfermedad de Parkinson del Reino Unido. 
Tabla 1: Criterios Clínicos de Diagnóstico de la Enfermedad de Parkinson (EP) del Banco de Cerebros de la Sociedad de

\section{Enfermedad de Parkinson del Reino Unido}

\begin{tabular}{|c|c|c|}
\hline $\begin{array}{c}\text { Síntomas Esenciales para el } \\
\text { Diagnóstico del Síndrome } \\
\text { Parkinsoniano }\end{array}$ & $\begin{array}{c}\text { Criterios de Exclusión para el Diagnóstico de } \\
\text { la EP Idiopática }\end{array}$ & $\begin{array}{c}\text { Criterios que Apoyan el Diagnóstico de la EP } \\
\text { Idiopática (se requieren } 3 \text { o más para el } \\
\text { diagnóstico definitivo) }\end{array}$ \\
\hline $\begin{array}{l}\text { Bradicinesia (lentitud en la } \\
\text { iniciación del movimiento } \\
\text { voluntario, progresiva reducción } \\
\text { en velocidad y amplitud de } \\
\text { acciones repetitivas). }\end{array}$ & $\begin{array}{l}\text { Antecedentes de TCE repetido, de encefalitis, } \\
\text { crisis oculogiras; uso de neuroléptico al inicio } \\
\text { de los síntomas; más de un pariente afectado; } \\
\text { remisión sostenida. }\end{array}$ & $\begin{array}{l}\text { - } \text { Comienzo unilateral. } \\
\text { - } \text { Temblor de reposo. } \\
\text { - } \quad \text { Cuadro progresivo. } \\
\text { - Asimetría persistente que compromete más } \\
\text { el lado donde comenzó. }\end{array}$ \\
\hline $\begin{array}{l}\text { Por lo menos uno de los siguientes: } \\
\text { - Rigidez. } \\
\text { - Temblor de reposo de } 4 \text { a } 6 \\
\text { Hz. } \\
\text { - Inestabilidad postural no } \\
\text { causada por compromiso } \\
\text { visual, vestibular, } \\
\text { cerebeloso o propioceptivo }\end{array}$ & $\begin{array}{l}\text { Síntomas unilaterales luego de } 3 \text { años de } \\
\text { evolución: • Parálisis supranuclear de la mirada } \\
\text { - Signos cerebelosos } \bullet \text { Compromiso } \\
\text { autonómico temprano y severo • Demencia } \\
\text { precoz con trastornos amnésicos del lenguaje y } \\
\text { praxia • Signo de Babinski • Presencia de un } \\
\text { tumor cerebral o hidrocefalia comunicante en } \\
\text { la TAC • Falta de respuesta a dosis adecuadas } \\
\text { de levodopa (si se excluye malabsorción) } \\
\text { Exposición a MPTP. }\end{array}$ & $\begin{array}{l}\text { - Excelente respuesta }(70-100 \%) \text { a la } \\
\text { levodopa. } \\
\text { - Corea severa inducida por levodopa. } \\
\text { - Respuesta a la levodopa de } 5 \text { años o más. } \\
\text { - Curso clínico de } 10 \text { años o más. }\end{array}$ \\
\hline
\end{tabular}




\subsection{Parkinson y emociones}

La relación del Parkinson con las emociones ha sido relegada a un segundo plano, por detrás de los síntomas motores. Sin embargo, durante los últimos años está cobrando más importancia este aspecto debido a la alta prevalencia de trastornos psiquiátricos y a la afectación en la calidad de vida de los pacientes.

La sintomatología de los pacientes con EP o incluso el sólo hecho de ser diagnosticado de esta enfermedad, invade al sujeto de diversas emociones de acuerdo a sus conocimientos y vivencia personal. Estas emociones van a influir sobre la salud de forma positiva o negativa, ya que por sus propiedades motivacionales, tienen capacidad para modificar la conducta de un sujeto. Las emociones negativas más importantes y que producen una experiencia emocional desagradable, son la ansiedad, la ira y la tristezadepresión ${ }^{(22)}$.

El impacto emocional ante el diagnóstico de la enfermedad precisará un período de aceptación por parte del paciente y de la familia, y posteriormente deberán ir adaptándose a la aparición de los diversos síntomas que influirán en su entorno familiar, social y laboral ${ }^{(23)}$. Durante todo este proceso es necesaria una buena orientación y apoyo psicológico para evitar aparición de trastornos psiquiátricos importantes y mejorar su calidad de vida.

Los síntomas que presenta el paciente van a influir en su vida diaria, volviéndose progresivamente dependiente ya que en los casos avanzados aparecen dificultades para el aseo personal, para vestirse o para la comunicación verbal y no verbal. En estos casos, muchos pacientes se abandonan y se dejan llevar por emociones negativas.

Otro aspecto importante a tener en cuenta es que existen casos en que el paciente presenta ansiedad o depresión en el período premotor de la enfermedad los cuales podrían empeorar con el diagnóstico y evolución de la enfermedad.

Es importante que el paciente mantenga una actitud positiva, reconociendo sus avances y logros, y buscando ayuda cuando lo necesite. Además, la presencia de ansiedad o 
depresión pueden incrementar los síntomas motores del Parkinson, lo que a su vez incrementará la ansiedad y/o depresión.

\subsubsection{Depresión}

La depresión es el trastorno neuropsiquiátrico más frecuente en la EP, variando su prevalencia según los diversos estudios y criterios empleados desde 2.7 a 90\%. Según la revisión sistemática de la depresión en la EP realizada por Reijnders et al. sobre un total de 36 artículos, encontraron que la prevalencia de trastorno depresivo mayor era del $17 \%$, de depresión menor del $22 \%$ y de distimia del 13\%, sin embargo, presentaban mayor prevalencia aquellos estudios que habían utilizado cuestionarios estructurados ${ }^{(24)}$.

En la etiología de la depresión existen muchas teorías. Algunos autores consideran que la depresión es una manifestación directa del daño cerebral o de la deficiencia de dopamina, noradrenalina y serotonina a lo que habría que sumar el efecto iatrogénico de ciertos fármacos y el estrés psicosocial debido a la enfermedad ${ }^{(25)}$.

El diagnóstico de la depresión, especialmente cuando va seguido de apatía, no siempre es fácil ya que puede haber superposición de síntomas psicopatológicos con manifestaciones clínicas de la EP, como la bradicinesia y la hipomimia. Para el diagnóstico de depresión es preciso la existencia de síntomas afectivos: tristeza, anhedonia, falta de motivación, sentimientos de culpa, derrotismo... En comparación con la depresión mayor primaria, los pacientes con EP presentan menor sentimiento de culpa, menos pensamientos autodestructivos y menor riesgo de suicidio. Son en cambio más frecuentes la angustia, crisis de ansiedad, fatiga, indecisión y anhedonia ${ }^{(25)}$.

La relación de la sintomatología depresiva con la gravedad de la EP es contradictoria, habiendo estudios que indican una relación directa ${ }^{(26)}$ y otros no encuentran relación ${ }^{(27)}$. Lo que en sí coinciden los estudios es que la depresión 
se relaciona con el deterioro de la capacidad de realizar las actividades de la vida diaria.

La fatiga es un síntoma principal, no específico, que se ha observado en diversas enfermedades sistémicas como en la EP y es uno de los síntomas más molestos que presentan. La depresión y las alteraciones del sueño se suelen considerar factores precipitantes de la fatiga, aunque el solapamiento entre los síntomas motores de parkinsonismo y las somatizaciones hace de éste un tema controvertido. La fatiga se considera un síntoma mayor en la depresión aunque en la EP la deficiencia dopaminérgica que afecta a los circuitos de los ganglios basales y el lóbulo frontal podría provocarla. En un estudio realizado con 40 pacientes, se estudió la posible relación de la fatiga con la implicación de mecanismos neuroinflamatorios observándose un incremento de las células NK en la EP, niveles de IL-1 $\beta$ aumentados y niveles de IL-1Ra antiinflamatoria, significativamente disminuidos; sin embargo no se puede dar una relación estadísticamente significativa $^{(28)}$.

\subsubsection{Ansiedad}

Los trastornos de ansiedad son también frecuentes en la EP y muchas veces se presentan antes de los síntomas motores. La ansiedad se puede manifestar con ataques de pánico, fobias o trastorno de ansiedad generalizada. Algunos estudios sugieren que los síntomas ansiosos y depresivos se relacionan principalmente con síntomas motores del lado izquierdo, aunque estos hallazgos son controversiales ${ }^{(29,30)}$. Su prevalencia varía según la metodología de los estudios realizados y puede coexistir con la depresión ${ }^{(31)}$.

Su fisiopatología se desconoce pero su coexistencia con la depresión o con los períodos de inmovilidad (off) de la enfermedad sugiere la participación central de neurotransmisores. Anormalidades en la norepinefrina, serotonina, dopamina y el ácido $\gamma$ aminobutírico podrían estar relacionados, aunque no hay datos concluyentes. La dopamina inhibe la actividad del locus coeruleus y la pérdida 
de la inhibición dopaminérgica podría explicar la elevada prevalencia de los trastornos de ansiedad en los pacientes con la enfermedad ${ }^{(32)}$.

Menza et al. estudiaron la relación entre las mutaciones del transportador de la serotonina y la enfermedad de Parkinson ${ }^{(33)}$. Encontraron que los pacientes con enfermedad de Parkinson portadores del alelo corto del transportador de serotonina presentaban scores más elevados de ansiedad y depresión que aquellos que no portaban dicha mutación. Ésto, junto con la asociación de la enfermedad de Parkinson a una personalidad premórbida con rasgos ansiosos ${ }^{(34)}$ y a la elevada prevalencia de ansiedad y depresión en los familiares con enfermedad de Parkinson (35), sugieren que los factores genéticos pueden formar parte de la patogénesis de la enfermedad ${ }^{(36)}$.

Algunos pacientes piden ayuda psicológica cuando experimentan ansiedad social. En algunos casos, esta ansiedad social lleva a un temor exagerado y persistente ante situaciones de relación social con miedo excesivo a la crítica por parte de los demás; todo esto les llevaría a evitar estas situaciones ocasionando fobia social. Se presenta en aproximadamente uno de cada diez pacientes con $\mathrm{EP}^{(25)}$.

Dissanayaka et al. estudiaron diversos factores relacionados con la ansiedad y la EP en 79 pacientes ${ }^{(37)}$. Encontraron que una EP más grave y el empeoramiento para la realización de las actividades diarias estaban directamente relacionadas con la sintomatología ansiosa. No estaba claro si la disminución en la capacidad para realizar actividades aumentó la ansiedad o si la ansiedad se tradujo en una disminución de las actividades básicas de la vida diaria. Las puntuaciones del Parkinson's Disease Questionnaire-8 (PDQ-8) sugirieron que la ansiedad estaba relacionada con la mala calidad de vida. Por el contrario, no encontraron relación con la dosis de Levodopa. 


\subsection{Calidad de Vida}

La Organización Mundial de la Salud (OMS) define la calidad de vida de forma multidimensional, en función de la manera en que el individuo percibe el lugar que ocupa en el entorno cultural y en el sistema de valores en que vive, así como en relación con sus objetivos, expectativas, criterios y preocupaciones. Todo ello matizado, por supuesto, por su salud física, su estado psicológico, su grado de independencia, sus relaciones sociales, los factores ambientales y sus creencias personales ${ }^{(38)}$.

No obstante, no existe un consenso universal sobre el concepto de calidad de vida ya que su definición puede basarse en diferentes enfoques. Debido a esto fue acuñado el término «calidad de vida relacionada con la salud (CVRS)» para nombrar únicamente los aspectos de la vida relacionados con la salud, la enfermedad y los tratamientos. La utilización básica del concepto de calidad de vida relacionada con la salud se inició en EE.UU. hace unos 30 años, con la confluencia de dos líneas de investigación: una, la de la investigación clínica de la medición del «estado funcional» y otra, la de la investigación psicológica del bienestar y de la salud mental ${ }^{(39)}$.

La OMS realizó además la Clasificación Internacional del Funcionamiento, de la Discapacidad y de la Salud (CIF) con la que logra una mayor comprensión del funcionamiento, la discapacidad y la salud. De esta forma pretende establecer un lenguaje común para describir la salud y los estados relacionados con ella y permitir la comparación de datos entre países, entre disciplinas sanitarias, entre los servicios, y en diferentes momentos a lo largo del tiempo. La CIF no clasifica personas, sino que describe la situación de cada persona dentro de un conjunto de dominios de la salud o dominios "relacionados con la salud". Además, la descripción siempre se hace en el contexto de los factores ambientales y personales ${ }^{(40)}$.

La preservación de la calidad de vida, a través de la prevención y el tratamiento de las enfermedades, es uno de los objetivos de la práctica médica, lo cual cobra mayor relevancia en los pacientes que padecen patologías crónicas como la enfermedad de Parkinson ${ }^{(41)}$. 
En la enfermedad de Parkinson, debido a que ésta tiene un alto impacto en la vida de los afectados y la de sus familiares, se da un deterioro progresivo de la calidad de vida a medida que progresa la enfermedad ${ }^{(42-44)}$. Hace años, se creía que esto se debía a la presencia de síntomas motores, sin embargo, se ha visto que la sintomatología ansiosodepresiva juega un rol importante en este aspecto ${ }^{(45)}$.

Existen diversas escalas para medir la calidad de vida en la EP, siendo la primera "The Parkinson's Disease Questionnaire" (PDQ-39). Comprende 39 preguntas con cinco opciones diferentes de respuesta relacionadas con la frecuencia de la manifestación de la enfermedad. Esta escala es la más usada en los estudios, existiendo la versión reducida que consta de 8 preguntas (PDQ-8) ${ }^{(46)}$.

La importancia de la evaluación de la CVRS se ha relacionado con el hecho de que el estado de salud percibido por el paciente es predictor de riesgos y de mortalidad, se asocia al consumo y demanda de recursos sanitarios, contribuye a la evaluación de resultados en investigación, y guía la toma de decisiones en la práctica clínica ${ }^{(47)}$. 


\section{HIPÓTESIS}




\section{HIPÓTESIS}

\subsection{Justificación de la hipótesis}

La enfermedad de Parkinson, al ser una patología crónica en la que el deterioro físico es progresivo e invalidante, conlleva la necesidad de una atención integral del paciente. Esta atención no se debe centrar sólo en los aspectos físicos, sino también en los aspectos emocionales y existenciales que se suscitan en el paciente y que pueden influir también en su calidad de vida.

En los últimos años recién se está dando la importancia debida a los síntomas no motores de la EP (autonómicos, sensoriales, cognitivos y neuropsiquiátricos) ${ }^{(29)}$ dentro de los que destacan por su frecuencia, los síntomas neuropsiquiátricos.

La sintomatología neuropsiquiátrica se encuentra tanto en las etapas tempranas como avanzadas de la enfermedad ${ }^{(48)}$, encontrándose porcentajes muy variables de prevalencia de depresión $(2.7-90 \%)^{(49)}$ y ansiedad $(6-55 \%)^{(50)}$, según la metodología realizada.

En este contexto, el estudio de la sintomatología ansioso-depresiva es de vital importancia ya que la presencia de estos síntomas afectaría la salud global del paciente, con efectos negativos en la dinámica familiar y en sus relaciones interpersonales, causando un impacto negativo en la calidad de vida del paciente. Para ayudar a su diagnóstico, existen diversos cuestionarios centrados en la ansiedad y la depresión, de entre los que destacan el Cuestionario de Ansiedad Estado - Rasgo (STAI) y el Inventario de Depresión de Beck - II (BDI-II).

Por otro lado, debido a que la diversidad de síntomas asociados a la enfermedad afecta a la funcionalidad y a las expectativas del individuo sobre su bienestar físico, social y mental; es importante analizar cómo ha afectado la EP en la calidad de vida de quienes la padecen. Para este análisis, la escala más usada es el Cuestionario de Calidad de Vida en Enfermedad de Parkinson (PDQ-39) en la que se abarcan diversos dominios de salud que ayudan a entender de forma global, el impacto de esta enfermedad en la vida de los pacientes. 


\subsection{Hipótesis de trabajo}

1) Los pacientes con enfermedad de Parkinson presentan características sociodemográficas que influyen en su sintomatología síntomas ansioso-depresiva.

- Hipótesis nula: Los síntomas ansioso-depresivos en la Enfermedad de Parkinson están influenciados por variables sociodemográficas, tales como la edad, el sexo, el estado civil y el sector de trabajo.

- Hipótesis alternativa: Los síntomas ansioso-depresivos en la Enfermedad de Parkinson no están influenciados por variables sociodemográficas, tales como la edad, el sexo, el estado civil y el sector de trabajo.

2) Los pacientes con Enfermedad de Parkinson presentan características clínicas que influyen en su sintomatología ansioso-depresiva.

- Hipótesis nula: Los síntomas ansioso-depresivos en la Enfermedad de Parkinson están influenciados por variables clínicas, tales como el antecedente familiar de Enfermedad de Parkinson, el tiempo de evolución de la enfermedad, el estadío evolutivo y el número de tratamientos antiparkinsonianos que recibe.

- Hipótesis alternativa: Los síntomas ansioso-depresivos en la Enfermedad de Parkinson no están influenciados por variables clínicas, tales como el antecedente familiar de Enfermedad de Parkinson, el tiempo de evolución de la enfermedad, el estadío evolutivo y el número de tratamientos antiparkinsonianos que recibe. 
3) Los pacientes con Enfermedad de Parkinson presentan alteración en la calidad de vida relacionada con la salud.

- Hipótesis nula: Los pacientes con diagnósticos de enfermedad de Parkinson no presentan alteración en la calidad de vida relacionada con la salud.

- Hipótesis alternativa: Los pacientes con diagnósticos de enfermedad de Parkinson sí presentan deterioro de su calidad de vida relacionada con la salud.

4) Los pacientes con Enfermedad de Parkinson que presentan además sintomatología ansioso-depresiva tienen una peor percepción de su calidad de vida.

- Hipótesis nula: La presencia de sintomatología ansioso-depresiva en la enfermedad de Parkinson no influye de forma relevante en el deterioro de la calidad de vida relacionada con la salud.

- Hipótesis alternativa: La presencia de sintomatología ansioso-depresiva asociada a la enfermedad de Parkinson influye en la calidad de vida relacionada con la salud del paciente. 
QBJETIXOS 


\section{OBJETIVOS DEL ESTUDIO}

\subsection{Objetivo general}

Establecer la prevalencia de la clínica ansioso-depresiva y determinar su impacto en la calidad de vida relacionada con la salud en los pacientes con Enfermedad de Parkinson.

\subsection{Objetivos específicos}

- Establecer la prevalencia de la clínica ansioso-depresiva en los pacientes con Enfermedad de Parkinson inscritos en la "Asociación Parkinson Asturias" a Marzo/2017 y determinar su relación con variables clínicas y sociodemográficas.

- Determinar el impacto de la clínica ansioso-depresiva en la calidad de vida relacionada con la salud en los pacientes con Enfermedad de Parkinson inscritos en la "Asociación Parkinson Asturias” a Marzo/2017.

- Determinar la relación de la calidad de vida relacionada con la salud con las variables clínicas y sociodemográficas. 
METOROLOGÍA 


\section{METODOLOGÍA}

\subsection{Diseño del estudio}

Se trata de un estudio observacional, descriptivo y de corte transversal.

\subsection{Población de estudio}

Pacientes con diagnóstico de EP que acudían a las diversas sedes de la "Asociación Parkinson Asturias" al mes de Marzo 2017.

Se contaba con una población de pacientes inscritos de 367, de los cuales acudían a la fecha de corte del estudio 203 pacientes con enfermedad EP. Participaron de forma voluntaria en el estudio 104 pacientes, siendo excluidos 9 por presentar deterioro cognitivo avanzado, déficit sensorial/motor importante o por haber rellenado las encuestas de forma incompleta. La población de estudio efectiva fue de 95 pacientes.

\subsection{Criterios de inclusión}

- Pacientes mayores de edad que acudían a la "Asociación Parkinson Asturias" durante el mes de Marzo 2017.

- Tener el diagnóstico de enfermedad de Parkinson idiopática de acuerdo con los criterios del Banco de Cerebros del Reino Unido.

- Obtención del consentimiento informado del paciente.

\subsection{Criterios de exclusión}

- Pacientes menores de edad.

- Pacientes incapaces de cumplimentar completamente los cuestionarios autoadministrados.

- Deterioro cognitivo del paciente valorado por el Mini-Examen Cognoscitivo (MEC-30): Puntuación <12 (demencia moderada) 
- Pacientes con incapacidad para leer y escribir.

- Pacientes con historia de psicosis.

- Pacientes con enfermedad concomitante en fase terminal o en tratamiento paliativo.

\subsection{Variables a estudio}

\section{- Variables sociodemográficas:}

- Edad del paciente (años).

- Género del paciente: Masculino o femenino.

- Estado civil del paciente: Soltero(a), casado(a), viudo(a) y divorciado(a). Posteriormente se los agrupó en casados y no casados. Este último incluye a: solteros(as), viudos(as) y divorciados(as).

- Nivel educativo: Se consideró The International Standard Classification of Education (ISCED):

$0 \quad$ Educación infantil.

1 Educación primaria.

2 Educación secundaria.

3 Bachillerato y ciclos formativos de grado medio.

4 Cursos de orientación universitaria.

5 Enseñanza universitaria.

6 Ciclos formativos de grado superior

7 Maestría, licenciatura o equivalente.

$8 \quad \mathrm{PhD} /$ Doctorado.

Posteriormente se agruparon los datos en: Sin instrucción (ISCED 0), primaria (ISCED 1), secundaria (ISCED 2,3 y 4) y superior (ISCED $5,6,7$ y 8$)$.

- Sector de trabajo: Primario, industrial, servicios y ama de casa. 


\section{- Variables clínicas:}

- Edad de inicio de la EP: $<50$ años y $\geq 50$ años.

- Tiempo desde el diagnóstico de enfermedad de Parkinson expresada en años. Se agruparon los valores en: $<3$ años, 3- 5 años, 6-10 años y $>10$ años.

- Estadificación de la gravedad de la enfermedad de Parkinson (Clasificación por Estadíos de Hoehn y Yahr) (Ver Anexo 2).

- Presencia de deterioro cognitivo y/o dependencia (Mini-Examen Cognoscitivo. MEC-30) (Ver anexo 3).

- Tratamientos recibidos para la EP por vía oral, incluyendo L-Dopa y derivados.

- Tratamientos avanzados para la EP (bomba de Apomorfina, bomba de Duodopa o cirugía de estimulación cerebral profunda).

- Otras enfermedades médicas.

- Diagnóstico previo de depresión, ansiedad u otra patología de salud mental.

- Otros tratamientos recibidos: Derivados opioides, antidepresivos, antipsicóticos, benzodiacepinas/análogos de benzodiacepinas (referidos en el estudio como ansiolíticos) y otros tratamientos médicos.

- Valoración de la calidad de vida según el el Cuestionario de Calidad de Vida en Enfermedad de Parkinson (PDQ-39) (Ver anexo 4).

- Presencia de sintomatología ansiosa y/o depresiva en el momento del estudio.

Las variables se explican en la tabla $\mathrm{N}^{0} 2$.

\subsection{Intervención}

Se acudió a las diversas sedes de la "Asociación Parkinson Asturias" para explicar a los socios las características del estudio y pedir su colaboración voluntaria. Se acordó con los participantes del estudio fechas para la entrevista y para la aplicación de los cuestionarios. 
Tabla 2: Variables de estudio.

\begin{tabular}{|c|c|c|c|}
\hline $\begin{array}{l}\text { Nombre de la } \\
\text { variable }\end{array}$ & Definición operativa & $\begin{array}{l}\text { Tipo de } \\
\text { variable }\end{array}$ & Categorías \\
\hline $\begin{array}{l}\text { Presencia de } \\
\text { sintomatología } \\
\text { depresiva }\end{array}$ & $\begin{array}{l}\text { Presencia o ausencia de depresión } \\
\text { según las puntuaciones obtenidas } \\
\text { en el BDI-II }\end{array}$ & $\begin{array}{l}\text { Cualitativa } \\
\text { dicotómica }\end{array}$ & $\begin{array}{l}\text { - Sí: presencia de puntuaciones en el BDI-II } \geq 14 \text {. } \\
\text { - No: presencia de puntuaciones en el BDI-II }<14 \text {. }\end{array}$ \\
\hline $\begin{array}{l}\text { Estratificación de } \\
\text { la depresión }\end{array}$ & $\begin{array}{l}\text { Presencia de depresión según la } \\
\text { clasificación de Beck et al. de } \\
1998 .\end{array}$ & $\begin{array}{l}\text { Cualitativa } \\
\text { ordinal. }\end{array}$ & $\begin{array}{l}\text { - Sin depresión: presencia de puntuaciones en el BDI-II <14. } \\
\text { - Depresión leve: presencia de puntuaciones en el BDI-II 14-19. } \\
\text { - Depresión moderada: presencia de puntuaciones en el BDI-II 20-28. } \\
\text { - Depresión grave: presencia de puntuaciones en el BDI-II 29-63. }\end{array}$ \\
\hline $\begin{array}{l}\text { Presencia de } \\
\text { sintomatología } \\
\text { ansiosa }\end{array}$ & $\begin{array}{l}\text { Presencia de puntuaciones de } \\
\text { STAI } / \mathrm{R}>\text { p50 }\end{array}$ & $\begin{array}{l}\text { Cualitativa } \\
\text { dicotómica }\end{array}$ & $\begin{array}{l}\text { - Sí: Presencia de puntuaciones de STAI/R >p50. } \\
\text { - No: Presencia de puntuaciones de STAI/R } \leq \text { p50. }\end{array}$ \\
\hline Calidad de vida & $\begin{array}{l}\text { Según puntuaciones obtenidas en } \\
\text { el PDQ-39 }\end{array}$ & Cuantitativa & Mayores puntuaciones, peor calidad de vida. \\
\hline
\end{tabular}




\begin{tabular}{|c|c|c|c|}
\hline $\begin{array}{c}\text { Nombre de la } \\
\text { variable }\end{array}$ & Definición operativa & $\begin{array}{l}\text { Tipo de } \\
\text { variable }\end{array}$ & Categorías \\
\hline \multicolumn{4}{|l|}{$\begin{array}{l}\text { Variables } \\
\text { sociodemográficas: }\end{array}$} \\
\hline Sexo & $\begin{array}{l}\text { Según refiera la persona } \\
\text { encuestada }\end{array}$ & $\begin{array}{l}\text { Cualitativa } \\
\text { nominal }\end{array}$ & $\begin{array}{l}\text { - Mujer. } \\
\text { - Varón. }\end{array}$ \\
\hline Estado civil & $\begin{array}{l}\text { Según refiera la persona } \\
\text { encuestada }\end{array}$ & $\begin{array}{l}\text { Cualitativa } \\
\text { nominal }\end{array}$ & $\begin{array}{l}\text { - Soltero(a). } \\
\text { - Casado(a). } \\
\text { - Viudo(a) } \\
\text { - Divorciado(a). }\end{array}$ \\
\hline Sector de trabajo & $\begin{array}{l}\text { Según refiera la persona } \\
\text { encuestada }\end{array}$ & $\begin{array}{l}\text { Cualitativa } \\
\text { ordinal }\end{array}$ & $\begin{array}{l}\text { - Primario. } \\
\text { - Industrial. } \\
\text { - Servicios. } \\
\text { - Ama de casa. }\end{array}$ \\
\hline
\end{tabular}




\begin{tabular}{|c|c|c|c|}
\hline $\begin{array}{l}\text { Nombre de la } \\
\text { variable }\end{array}$ & Definición operativa & $\begin{array}{l}\text { Tipo de } \\
\text { variable }\end{array}$ & Categorías \\
\hline \multicolumn{4}{|l|}{ Variables clínicas: } \\
\hline $\begin{array}{l}\text { Antecedente } \\
\text { familiar de EP }\end{array}$ & $\begin{array}{l}\text { Según refiera la persona } \\
\text { encuestada }\end{array}$ & $\begin{array}{l}\text { Cualitativa } \\
\text { nominal }\end{array}$ & $\begin{array}{l}\text { - Sí. } \\
\text { - No. } \\
\text { - No sabe. }\end{array}$ \\
\hline $\begin{array}{l}\text { Edad de inicio de } \\
\text { EP }\end{array}$ & $\begin{array}{l}\text { Según refiera el paciente como } \\
\text { edad de inicio de síntomas }\end{array}$ & $\begin{array}{l}\text { Cuantitativa } \\
\text { ordinal }\end{array}$ & $\begin{array}{l}-<50 \text { años. } \\
-\geq 50 \text { años. }\end{array}$ \\
\hline $\begin{array}{l}\text { Tiempo de } \\
\text { evolución EP }\end{array}$ & $\begin{array}{l}\text { Según refiera la persona } \\
\text { encuestada, expresada en años. }\end{array}$ & $\begin{array}{l}\text { Cuantitativa } \\
\text { continua }\end{array}$ & Según datos indicados por el paciente. \\
\hline $\begin{array}{l}\text { Gravedad de la } \\
\text { EP }\end{array}$ & $\begin{array}{l}\text { Se agrupó según la clasificación } \\
\text { por Estadíos de Hoehn y Yahr }\end{array}$ & $\begin{array}{l}\text { Cualitativa } \\
\text { ordinal. }\end{array}$ & $\begin{array}{l}\text { - Estadío 0: No hay signos de enfermedad. } \\
\text { - Estadío 1: Enfermedad exclusivamente unilateral. } \\
\text { - Estadío 1.5: Afectación unilateral y axial. } \\
\text { - Estadío 2.0: Afectación bilateral sin alteración del equilibrio. } \\
\text { - Estadío 2.5: Afectación bilateral leve con recuperación en la prueba } \\
\text { de retropulsión. } \\
\text { - Estadío 3.0: Afectación bilateral leve a moderada; cierta inestabilidad } \\
\text { postural, pero físicamente independiente. } \\
\text { - Estadío 4.0: Incapacidad grave; aún capaz de caminar o de } \\
\text { permanecer en pie sin ayuda. } \\
\text { - Estadío 5.0: Permanece en silla de ruedas/encamado si no tiene ayuda }\end{array}$ \\
\hline
\end{tabular}




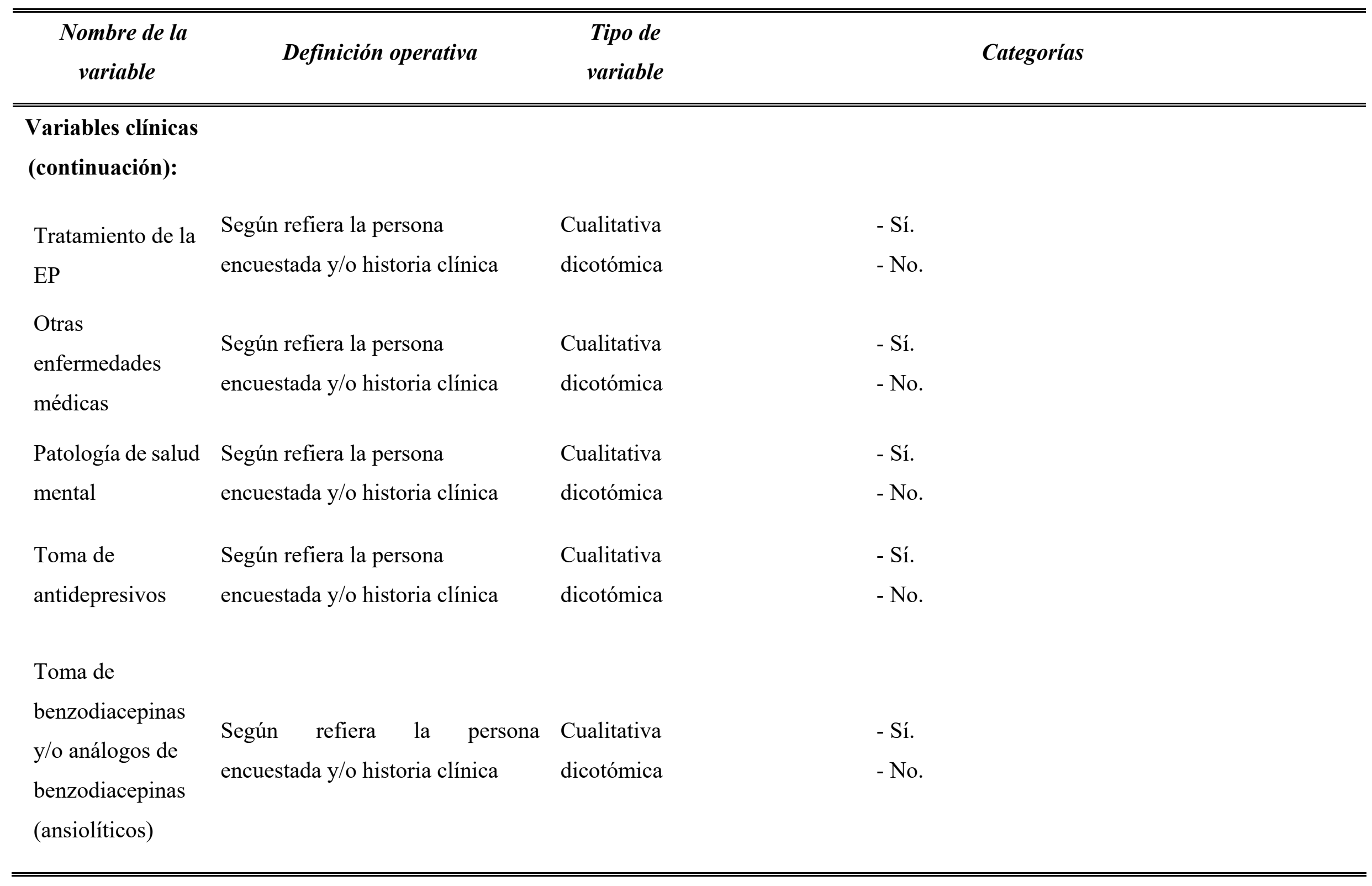




\begin{tabular}{|c|c|c|c|}
\hline $\begin{array}{c}\text { Nombre de la } \\
\text { variable }\end{array}$ & Definición operativa & $\begin{array}{l}\text { Tipo de } \\
\text { variable }\end{array}$ & Categorías \\
\hline \multicolumn{4}{|c|}{$\begin{array}{l}\text { Variables clínicas } \\
\text { (continuación): }\end{array}$} \\
\hline $\begin{array}{l}\text { Otros } \\
\text { tratamientos } \\
\text { médicos }\end{array}$ & $\begin{array}{l}\text { Según refiera la persona } \\
\text { encuestada y/o historia clínica }\end{array}$ & $\begin{array}{l}\text { Cualitativa } \\
\text { dicotómica }\end{array}$ & $\begin{array}{l}\text { - Sí. } \\
\text { - No. }\end{array}$ \\
\hline $\begin{array}{l}\text { Deterioro } \\
\text { cognitivo }\end{array}$ & $\begin{array}{l}\text { Presencia o ausencia de deterioro } \\
\text { cognitivo según puntuaciones } \\
\text { obtenidas en el MEC-30 } \\
\text { corregidas por nivel educativo y } \\
\text { edad. }\end{array}$ & $\begin{array}{l}\text { Cualitativa } \\
\text { ordinal }\end{array}$ & $\begin{array}{l}\text { Normal: según puntuaciones obtenidas en el MEC-30 corregidas por } \\
\text { nivel educativo y edad. } \\
\text { Sospecha de patología: según puntuaciones obtenidas en el MEC-30 } \\
\text { corregidas por nivel educativo y edad. } \\
\text { Deterioro cognitivo: según puntuaciones obtenidas en el MEC-30 } \\
\text { corregidas por nivel educativo y edad. }\end{array}$ \\
\hline
\end{tabular}


Se realizó una entrevista individual con cada paciente donde fueron preguntados sobre las variables demográficas e historia médica-psiquiátrica relevante. Posteriormente se les aplicó el Mini-Examen Cognoscitivo (MEC-30), descartándose aquellos pacientes que obtuvieron una puntuación $<12$ (demencia moderada), los que presentaban problemas para leer o escribir, que tenían diagnóstico de psicosis o que se encontraban con una enfermedad concomitante en fase terminal o en tratamiento paliativo. Se utilizó la Clasificación por Estadíos de Hoehn y Yahr para medir el estadío de la enfermedad de Parkinson (ver anexo 2).

Se les explicó en un salón grupal cómo completar los cuestionarios de Beck-II, de Ansiedad Estado-Rasgo (STAI) y el Parkinson Disease Questionnaire 39 (PDQ-39). Cualquier duda que pudiera surgir durante la respuesta a los cuestionarios fue resuelta en ese mismo momento. Además, se puso un código a cada paciente para garantizar su anonimato.

Los cuestionarios utilizados fueron:

El Cuestionario de Ansiedad Estado-Rasgo (STAI) para valorar la presencia de ansiedad (ver anexo 5): Consta de 40 preguntas divididas en 2 subescalas: 1) Ansiedad Estado (A/E) que hace referencia a una condición emocional transitoria del organismo, caracterizada por sentimientos subjetivos de tensión y aprensión y; 2) Ansiedad Rasgo (A/R) que hace referencia a la propensión ansiosa estable que hace percibir a las personas y las situaciones como amenazadoras, elevando así la ansiedad. Cada subescala está compuesta por 20 preguntas que tienen 4 alternativas cada una. Cada pregunta se puntúa en una escala tipo Likert de 0-3 según su carga y dirección hacia la ansiedad, existiendo 10 enunciados negativos de ansiedad en A/E y 7 en A/R. Para analizar la presencia de la clínica ansiosa en los pacientes, se utilizaron los datos obtenidos del STAI/R debido a que ve la permanencia de esta sintomatología durante el tiempo. La interpretación de los resultados se basó en la conversión a centiles, según la puntuación obtenida en el subtest y el sexo del paciente, tal como se recomienda en la adaptación española (ver anexo 6) ${ }^{(51)}$. No existe un punto de corte definido, variando el mismo en las 
diversas publicaciones ${ }^{(52-57)}$. En este estudio se consideró ansiedad elevada valores mayores del centil 50 58-62). Además, debido a la amplia variación de resultados se dividió la ansiedad en baja ( $\leq 25)$, media-baja ( $225-\mathrm{p} \leq 50)$, alta $(p>50-p \leq 75)$ y muy alta $(p>75)$.

- El Cuestionario de Depresión de Beck-II (BDI-II) para valorar la presencia de depresión (ver anexo 7): Consta de 21 preguntas con 4 alternativas cada una que se puntúan de 0 -3. Se consideró la clasificación de Beck et al. de 1998 para medir la depresión: sin depresión (0-13), depresión leve (14-19), depresión moderada (20-28) y depresión grave (29-63) ${ }^{(63)}$.

- El Cuestionario de Calidad de Vida en Enfermedad de Parkinson (PDQ-39) para medir la calidad de vida relacionada con la salud del paciente en sus diversas dimensiones (ver anexo 4). Se utilizó este cuestionario debido a que es el más utilizado en los estudios de investigación ${ }^{(64-66)}$. Consta de 39 preguntas divididas en 8 dimensiones (movilidad, actividades de la vida diaria, estado emocional, estigmatización de la enfermedad, soporte social, estado cognitivo, comunicación y malestar corporal o dolor). Cada pregunta recibe una puntuación de 1-4 según la frecuencia de los síntomas enunciados y se expresan en porcentaje. Posteriormente se utilizaron los resultados para obtener el Parkinson's Disease Summary Index (PDSI) el cual tiene una puntuación de 0-100 donde 0 es el estado de salud perfecto y 100 es el peor estado de salud.

- El Mini-Examen Cognoscitivo (MEC-30) para medir el deterioro cognitivo (ver anexo 3): consta de 30 ítems agrupados en 11 secciones que miden la orientación temporal, la orientación espacial, la fijación, la atención y cálculo, la memoria, la nominación, la repetición, la comprensión, la lectura, la escritura y el dibujo. Cada pregunta que se responda correctamente tiene una puntuación de 1. Los valores obtenidos se corrigieron por nivel educativo y edad. 


\subsection{Consideraciones éticas}

A lo largo del estudio se han respetado las normas éticas pertinentes ${ }^{(67,68)}$.

Se obtuvo la aprobación del proyecto por parte del Comité de Ética de la Investigación del Principado de Asturias (anexo 8).

Se obtuvo el consentimiento informando de todos los participantes. Se tomaron todas las medidas necesarias para garantizar el derecho a la intimidad de los encuestados. En todo momento se mantuvo el carácter confidencial de sus datos personales.

\subsection{Análisis estadístico}

Los datos fueron procesados informáticamente mediante una base de datos en formato Microsoft Excel. Posteriormente los datos fueron importados para su tratamiento estadístico al programa StataSE ${ }^{12}$, para analizar la ansiedad y la depresión y a $\mathrm{R}(\mathrm{R}$ development Core Team) versión 3.4.4, para analizar la calidad de vida.

Las variables cualitativas fueron expresadas mediante frecuencias absolutas y frecuencias relativas; esta última se expresó en porcentaje. Para las variables cuantitativas continuas se encontró la media junto con la desviación estándar (DS). Para el análisis bivariante se realizó tablas de contingencia, se calculó su probabilidad de error y su IC al 95\% correspondiente. Se expresó de forma gráfica mediante diagrama de barras o de sectores circulares aquellas variables que se creyeron convenientes para su mejor entendimiento. Para el análisis de la relación de la depresión y la ansiedad con el resto de variables se calculó el Odds Ratio (OR) mediante StataSE ${ }^{12}$ para ver mejor el grado de influencia de cada variable.

En el análisis de la calidad de vida, las relaciones entre variables cualitativas se valoraron con el test Chi Cuadrado de Pearson o con el test de Fisher, según se verificase o no la hipótesis sobre frecuencias esperadas. Las diferencias de variables cuantitativas entre dos grupos se estudiaron con el test $\mathrm{t}$ de Student o el test de Wilcoxon para muestras independientes, según se verificase o no la hipótesis de frecuencias esperadas. Si los 
grupos a comparar eran 3 o más, los test empleados fueron el Anova o el de KruskalWallis, según se cumpliesen las hipótesis de normalidad y homocedasticidad.

Posteriormente se realizaron análisis de regresión múltiple para ver la influencia de las diversas variables en las variables dependientes. En la primera parte, que estudia a la depresión y ansiedad como variables dependientes, se obtuvieron valores de OR y; en la segunda parte, que estudia a la calidad de vida como variable dependiente, se calculó el $\mathrm{R}^{2}$ ajustado para el PDSI y cada una de las dimensiones del PDQ-39. Se consideraron diferencias estadísticamente significativas aquellas que presentaban una probabilidad de error inferior al 5\% $(\mathrm{p}<0.05)$.

Por otro lado, se midió la consistencia interna de los cuestionarios mediante el Coeficiente alfa de Cronbach. Este coeficiente puede dar valores que oscilan entre 0 y 1 , siendo más confiable la prueba mientras más se aproxime a la unidad.

El Coeficiente Alfa de Cronbach encontrado en los cuestionarios fue mayor de 0.7 lo que indica una fuerte relación entre cada una de las preguntas de cada test (consistencia interna). Los valores obtenidos fueron: 0.857 para el BDI-II, 0.890 para el STAI-E y 0.861 para el STAI-R. Se analizó cada una de las dimensiones del PDQ-39 siendo todos los resultados satisfactorios (movilidad: 0.878 , actividades de la vida diaria: 0.885 , estado emocional: 0.856 , estigmatización de la enfermedad: 0.703 , soporte social: 0.701 , estado cognitivo: 0.702 , comunicación: 0.808 y dolor: 0.708 ). 
RESULTADOS 


\section{RESULTADOS}

\subsection{Descripción de la muestra}

La población estudiada fue de 95 pacientes compuesta por 56 varones (58.95\%) y 39 mujeres (41.05\%). Se recolectaron datos de pacientes de 4 sedes de la Asociación Parkinson Asturias (ver figura 1).

\section{Sedes de Asociación de Parkinson Asturias}

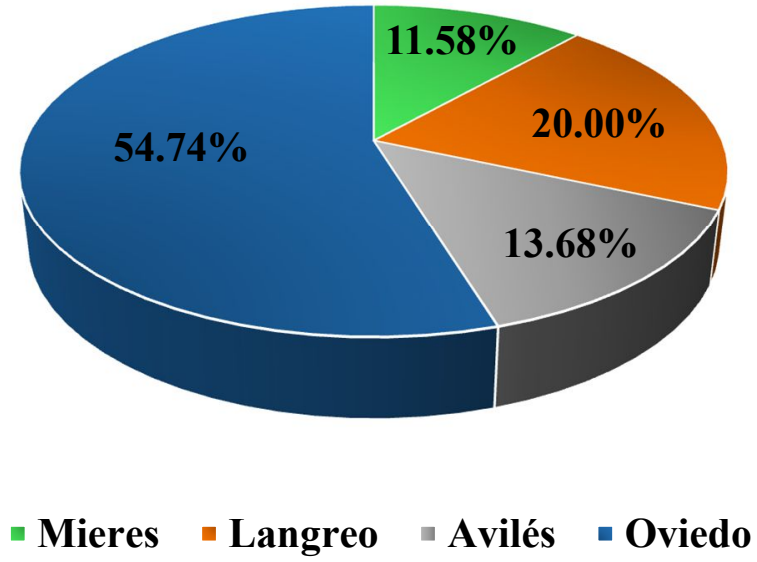

Figura 1: Pacientes de la Asociación de Parkinson Asturias según sedes estudiadas.

Figura que muestra la distribución delos pacientes según las sedes de la Asociación

Parkinson Asturias estudiadas. Se puede observar que la mayor parte de los pacientes pertenecía a la sede de Oviedo, que era también la más grande. 
La edad media fue de $70.43 \pm 8.02$ años para los varones y de $71.28 \pm 8.05$ años para las mujeres. Cuando se consideró la edad de inicio de la EP, la edad media fue de $61.4 \pm 4.48$ años para los pacientes con inicio temprano ( $<50$ años) y de $71.88 \pm 7.60$ años para los demás pacientes (inicio $\geq 50$ años). La mayoría estaba casado(a) (70.53\%) aunque al agrupar el estado civil por sexo, esta predominancia se mantenía en los varones (92.86\%) y cambiaba en las mujeres, en quienes predominaban las viudas/divorciadas (53.85\%).

Al analizar el sector de trabajo de los pacientes estudiados, se encontró que en ambos sexos la mayoría trabajaba o había trabajado en el sector servicios. El segundo en frecuencia en varones fue el sector industrial mientras que en las mujeres fue el de ama de casa $(\mathrm{p}<0.001)$.

Por otro lado, se realizó la valoración cognitiva encontrando que la mayor parte de la población presentó una puntuación del $M E C$-30, corregido por nivel educativo, dentro del rango normal (84.21\%).

Al analizar diferentes características de la EP, encontramos que la edad media de aparición de los síntomas fue de $62.63 \pm 10.36$ años $(62.71 \pm 10.29$ para los varones y $62.51 \pm 10.59$ para las mujeres), siendo el $10.53 \%$ de inicio temprano ( $<50$ años). No se encontró diferencias estadísticamente significativas por sexo en ambas variables. Tampoco se encontró diferencias significativas por sexo en el tiempo de evolución de la enfermedad ( $7.72 \pm 5.88$ en varones VS $8.78 \pm 7$ para mujeres; $p=0.652)$, el nivel educativo $(p=0.157)$, ni a la presencia de antecedentes familiares de EP $(p=0.919)$.

La gravedad de la EP según los estadíos de Hoehn y Yahr tuvo una distribución bimodal, siendo más frecuente el estadío 1.0 (26.32\%) seguido del estadío 3.0 (25.26\%), no encontrándose diferencias significativas por sexo $(\mathrm{p}=0.967)$. No hubo pacientes en el estadío 5.0. La figura 2 muestra la distribución de la gravedad de la EP según sexo.

La tabla 3 muestra un resumen de las características generales de la población estudiada según su sexo. 


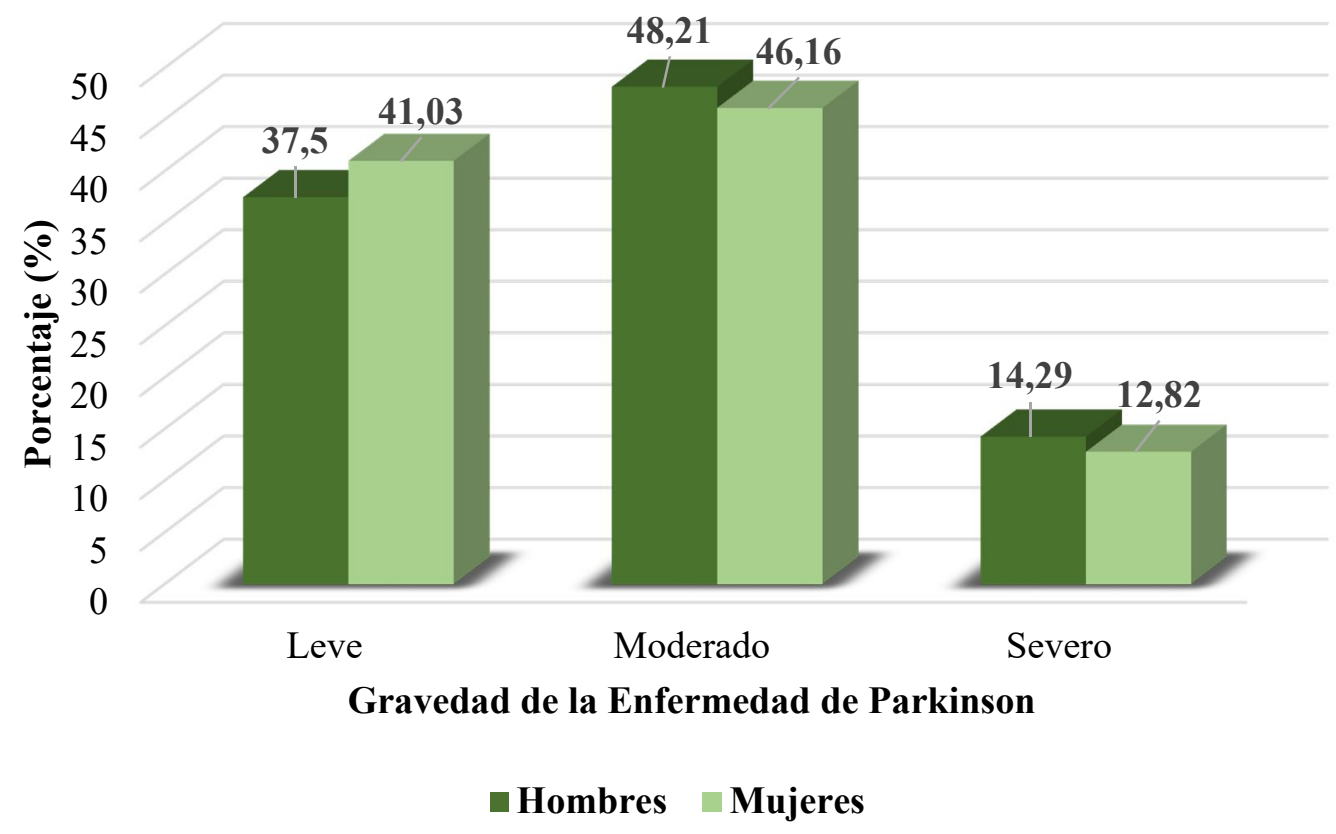

Figura 2: Distribución de la gravedad de la Enfermedad de Parkinson según sexo.

Diagrama de barras en el que se muestra la distribución de la gravedad de la enfermedad de Parkinson según el sexo del paciente. Leve: Estadíos 0, 1.0 y 1.5; moderado: 2.0, 2.5 y 3.0; severo: 4.0 y 5.0. Puede observarse una mayor prevalencia del nivel moderado. 
Tabla 3: Características generales de la población.

\begin{tabular}{|c|c|c|c|c|c|c|c|}
\hline \multirow{3}{*}{ Característica } & \multicolumn{6}{|c|}{ Sexo } & \multirow{3}{*}{ p-value } \\
\hline & \multicolumn{2}{|c|}{ Hombres } & \multicolumn{2}{|c|}{ Mujeres } & \multicolumn{2}{|c|}{ Total } & \\
\hline & $N$ & $\%$ & $N$ & $\%$ & $N$ & $\%$ & \\
\hline \multicolumn{8}{|l|}{ Estado Civil } \\
\hline Soltero/a & 1 & 4.21 & 3 & 7.69 & 4 & 4.21 & \multirow{3}{*}{$<0.001$} \\
\hline Casado(a) & 52 & 92.86 & 15 & 38.46 & 67 & 70.53 & \\
\hline Viudo(a)/divorciado(a) & 3 & 5.36 & 21 & 53.85 & 24 & 25.26 & \\
\hline \multicolumn{8}{|l|}{ Nivel Educativo } \\
\hline Ninguna & 4 & 7.14 & 1 & 2.56 & 5 & 5.26 & \multirow{4}{*}{0.157} \\
\hline Primaria & 19 & 33.93 & 21 & 53.85 & 40 & 42.11 & \\
\hline Secundaria & 16 & 28.57 & 11 & 28.21 & 27 & 28.42 & \\
\hline Superior & 17 & 30.36 & 6 & 15.38 & 23 & 24.21 & \\
\hline \multicolumn{8}{|l|}{ Sector de Trabajo } \\
\hline Primario & 11 & 19.64 & 0 & 0.00 & 11 & 11.58 & \multirow{4}{*}{$<0.001$} \\
\hline Industrial & 19 & 33.93 & 1 & 2.57 & 20 & 21.05 & \\
\hline Servicios & 26 & 46.43 & 20 & 51.28 & 46 & 48.42 & \\
\hline Ama de casa & 0 & 0.00 & 18 & 46.15 & 18 & 18.95 & \\
\hline \multicolumn{8}{|l|}{ Estado Cognitivo } \\
\hline Normal & 48 & 85.71 & 32 & 82.05 & 80 & 84.21 & \multirow{3}{*}{0.366} \\
\hline Sospecha de patología & 5 & 8.93 & 2 & 5.13 & 7 & 7.37 & \\
\hline Deterioro cognitivo & 3 & 5.36 & 5 & 12.82 & 8 & 8.42 & \\
\hline \multicolumn{8}{|l|}{ AF Parkinson } \\
\hline No & 37 & 66.07 & 26 & 66.67 & 63 & 66.32 & \multirow{3}{*}{0.919} \\
\hline $\mathrm{Si}$ & 17 & 30.36 & 11 & 28.21 & 28 & 29.47 & \\
\hline No sabe & 2 & 3.57 & 2 & 5.13 & 4 & 4.21 & \\
\hline \multicolumn{8}{|c|}{ Estadíos de Hoehn y Yahr } \\
\hline 0 & 2 & 3.57 & 1 & 2.56 & 3 & 3.16 & \multirow{8}{*}{0.967} \\
\hline 1.0 & 14 & 25.00 & 11 & 28.21 & 25 & 26.32 & \\
\hline 1.5 & 5 & 8.93 & 4 & 10.26 & 9 & 9.47 & \\
\hline 2.0 & 4 & 7.14 & 3 & 7.69 & 7 & 7.37 & \\
\hline 2.5 & 10 & 17.86 & 4 & 10.26 & 14 & 14.74 & \\
\hline 3.0 & 13 & 23.21 & 11 & 28.21 & 24 & 25.26 & \\
\hline 4.0 & 8 & 14.29 & 5 & 12.82 & 13 & 13.68 & \\
\hline 5.0 & 0 & 0.00 & 0 & 0.00 & 0 & 0.00 & \\
\hline
\end{tabular}

Al estudiar la presencia de otras enfermedades asociadas, se encontró que la mayor parte de la población estudiada tenía al menos una enfermedad orgánica asociada $(86.32 \%)$ de las cuales la más frecuente fue la patología cardiovascular $(41.05 \%)$. Se encontró 
diferencia significativa por sexo en la patología pulmonar que estuvo presente en el $16.07 \%$ de los varones y ausente en las mujeres estudiadas (ver tabla 4).

Con respecto a los antecedentes neuropsiquiátricos, 19 pacientes $(20.00 \%)$ ya tenían el diagnóstico de depresión al momento del estudio, 9 (9.47\%) de ansiedad y uno (1.1\%) presentaba ambos diagnósticos. Además, 20 pacientes $(21.05 \%)$ presentaban otro problema neuropsiquiátrico asociado, principalmente insomnio. Se encontró que el antecedente de depresión era más frecuente en mujeres con una diferencia estadísticamente significativa $(\mathrm{p}=0.029)$ (ver tabla 5$)$.

En este estudio se encontró que el $95.79 \%$ de los pacientes recibía algún tipo de tratamiento antiparkinsoniano. Terapias avanzadas junto a tratamiento oral las recibían el 11.58\%. El principal tratamiento oral recibido fue L-Dopa con un $81.05 \%$ de los participantes $(n=77)$. No se encontraron pacientes que usaran Bomba de Duodopa ni diferencias significativas entre el tipo de tratamiento cuando se analizaron por sexos (ver tablas 6 y 7$)$.

En la tabla 8 se resume el consumo de otro tipo de medicamentos, destacando un mayor consumo de antidepresivos y derivados opioides en mujeres ( $p=0.001$ y $p=0.014$ respectivamente).

Se evaluó la calidad mediante el PDQ-39 y el PDSI. Al analizar los resultados obtenidos en las diversas dimensiones del PDQ-39, se encontraron diferencias estadísticamente significativas $(\mathrm{p}<0.05)$ en las dimensiones de "comunicación" (centiles más elevados en los varones), "bienestar emocional”, “estado cognitivo" y "dolor” (centiles más elevados en las mujeres). No se encontraron diferencias significativas en relación al sexo en las dimensiones de "movilidad", "actividades de la vida diaria", "estigmatización" y "apoyo social”. El PDSI no mostró diferencias por sexos (ver tabla 9). 
Tabla 4: Presencia de comorbilidades.

\begin{tabular}{|c|c|c|c|c|c|c|c|}
\hline \multirow{3}{*}{ Otras Patologías Médicas } & \multicolumn{6}{|c|}{ Sexo } & \multirow{3}{*}{ p-value } \\
\hline & \multicolumn{2}{|c|}{ Hombres } & \multicolumn{2}{|c|}{ Mujeres } & \multicolumn{2}{|c|}{ Total } & \\
\hline & $N$ & $\%$ & $N$ & $\%$ & $N$ & $\%$ & \\
\hline Cardiovascular & 22 & 39.29 & 17 & 43.59 & 39 & 41.05 & 0.675 \\
\hline Pulmonar & 9 & 16.07 & 0 & 0.00 & 9 & 9.57 & 0.009 \\
\hline Digestivo & 3 & 5.36 & 1 & 2.56 & 4 & 4.21 & 0.505 \\
\hline Oncológica & 2 & 3.57 & 2 & 5.13 & 4 & 4.21 & 0.710 \\
\hline Otras & 38 & 67.86 & 30 & 76.92 & 68 & 71.58 & 0.335 \\
\hline
\end{tabular}

Tabla 5: Presencia de otros trastornos neuropsiquiátricos.

\begin{tabular}{|c|c|c|c|c|c|c|c|}
\hline \multirow{3}{*}{ Otros trastornos neuropsiquiátricos } & \multicolumn{6}{|c|}{ Sexo } & \multirow{3}{*}{$P$-value } \\
\hline & \multicolumn{2}{|c|}{ Hombres } & \multicolumn{2}{|c|}{ Mujeres } & \multicolumn{2}{|c|}{ Total } & \\
\hline & $N$ & $\%$ & $N$ & $\%$ & $N$ & $\%$ & \\
\hline Ansiedad & 5 & 8.93 & 4 & 10.26 & 9 & 9.47 & 0.828 \\
\hline Depresión & 7 & 12.50 & 12 & 30.77 & 19 & 20.00 & 0.029 \\
\hline Otros & 16 & 28.57 & 4 & 10.26 & 20 & 21.05 & 0.031 \\
\hline
\end{tabular}


Tabla 6: Distribución de tratamiento antiparkinsoniano.

\begin{tabular}{|c|c|c|c|c|c|c|c|}
\hline \multirow{3}{*}{$\begin{array}{c}\text { Tipos de tratamiento } \\
\text { Específico }\end{array}$} & \multicolumn{6}{|c|}{ Sexo } & \multirow{3}{*}{$P$-value } \\
\hline & \multicolumn{2}{|c|}{ Hombres } & \multicolumn{2}{|c|}{ Mujeres } & \multicolumn{2}{|c|}{ Total } & \\
\hline & $N$ & $\%$ & $N$ & $\%$ & $N$ & $\%$ & \\
\hline \multicolumn{8}{|l|}{ Oral } \\
\hline Sin fármacos & 2 & 3.57 & 2 & 5.13 & 4 & 4.21 & \multirow{5}{*}{0.588} \\
\hline 1 fármaco & 11 & 19.64 & 6 & 15.38 & 17 & 17.89 & \\
\hline 2 fármacos & 18 & 32.14 & 14 & 35.90 & 32 & 33.68 & \\
\hline 3 fármacos & 15 & 26.79 & 14 & 35.90 & 29 & 30.53 & \\
\hline$>3$ fámacos & 10 & 17.86 & 3 & 7.69 & 13 & 13.68 & \\
\hline \multicolumn{8}{|l|}{ Terapia avanzado } \\
\hline Ninguno & 50 & 89.29 & 34 & 87.18 & 84 & 88.42 & \multirow[t]{2}{*}{0.752} \\
\hline Sí & 6 & 10.71 & 5 & 12.82 & 11 & 11.58 & \\
\hline
\end{tabular}


Tabla 7: Tipos de tratamiento antiparkinsoniano.

\begin{tabular}{|c|c|c|c|c|c|c|c|}
\hline \multirow{3}{*}{ Tipos de tratamiento específico } & \multicolumn{6}{|c|}{ Sexo } & \multirow{3}{*}{$P$-value } \\
\hline & \multicolumn{2}{|c|}{ Hombres } & \multicolumn{2}{|c|}{ Mujeres } & \multicolumn{2}{|c|}{ Total } & \\
\hline & $N$ & $\%$ & $N$ & $\%$ & $N$ & $\%$ & \\
\hline \multicolumn{8}{|l|}{ Tratamiento Oral } \\
\hline L-dopa & 48 & 85.71 & 29 & 74.36 & 77 & 81.05 & 0.165 \\
\hline Anticolinérgicos & 1 & 1.79 & 1 & 2.56 & 2 & 2.11 & 0.795 \\
\hline Agonistas dopaminérgicos & 30 & 53.57 & 18 & 46.15 & 48 & 50.53 & 0.477 \\
\hline Inhibidores de la MAO-B & 28 & 50.00 & 26 & 66.67 & 54 & 56.84 & 0.107 \\
\hline Otros & 25 & 44.64 & 14 & 35.90 & 39 & 41.05 & 0.394 \\
\hline \multicolumn{8}{|l|}{ Terapias Avanzadas } \\
\hline Bomba de Apomorfina & 2 & 3.57 & 1 & 2.56 & 3 & 3.16 & 0.782 \\
\hline Cirugía & 4 & 7.14 & 4 & 10.26 & 8 & 8.42 & 0.591 \\
\hline
\end{tabular}


Tabla 8: Otros tratamientos.

\begin{tabular}{|c|c|c|c|c|c|c|c|}
\hline \multirow{3}{*}{ Otros tratamientos } & \multicolumn{6}{|c|}{ Sexo } & \multirow{3}{*}{ P-value } \\
\hline & \multicolumn{2}{|c|}{ Hombres } & \multicolumn{2}{|c|}{ Mujeres } & \multicolumn{2}{|c|}{ Total } & \\
\hline & $N$ & $\%$ & $N$ & $\%$ & $N$ & $\%$ & \\
\hline Tratamiento de deterioro cognitivo & 7 & 12.5 & 5 & 12.82 & 12 & 12.63 & 0.963 \\
\hline Derivados opioides & 0 & 0.00 & 4 & 10.26 & 4 & 4.21 & 0.014 \\
\hline Ansiolíticos & 25 & 44.64 & 12 & 30.77 & 37 & 38.95 & 0.173 \\
\hline Antidepresivos & 7 & 12.50 & 17 & 43.59 & 24 & 25.26 & 0.001 \\
\hline Antipsicóticos & 2 & 3.57 & 3 & 7.69 & 5 & 5.26 & 0.376 \\
\hline Otros tratamientos médicos & 44 & 78.57 & 37 & 94.87 & 81 & 82.26 & 0.027 \\
\hline
\end{tabular}


Tabla 9: Calidad de vida.

\begin{tabular}{|c|c|c|c|c|c|c|c|}
\hline \multirow{3}{*}{ TEST DE CALIDAD DE VIDA } & \multicolumn{6}{|c|}{ Sexo } & \multirow{3}{*}{ p-value } \\
\hline & \multicolumn{2}{|c|}{ Hombres } & \multicolumn{2}{|l|}{ Mujeres } & \multicolumn{2}{|l|}{ Total } & \\
\hline & Media (centil) & DS & Media (centil) & DS & Media (centil) & DS & \\
\hline \multicolumn{8}{|l|}{ PDQ-39 Dimensión } \\
\hline Movilidad & 31.21 & 21.17 & 35.26 & 26.80 & 32.87 & 23.59 & 0.414 \\
\hline Actividades de la vida diaria & 29.61 & 23.91 & 24.57 & 27.98 & 27.54 & 25.63 & 0.349 \\
\hline Bienestar emocional & 25.52 & 17.71 & 38.03 & 22.90 & 30.66 & 20.83 & 0.003 \\
\hline Estigmatización & 8.37 & 10.27 & 13.14 & 17.13 & 10.32 & 13.63 & 0.125 \\
\hline Apoyo Social & 21.88 & 23.81 & 17.09 & 21.54 & 19.91 & 22.91 & 0.320 \\
\hline Estado Cognitivo & 28.24 & 18.62 & 41.03 & 22.25 & 33.49 & 21.05 & 0.003 \\
\hline Comunicación & 24.85 & 23.00 & 13.46 & 19.55 & 20.18 & 22.27 & 0.013 \\
\hline Dolor & 30.80 & 22.75 & 45.30 & 21.27 & 36.75 & 23.17 & 0.002 \\
\hline PDSI & 25.06 & 12.94 & 28.49 & 14.53 & 26.47 & 13.64 & 0.231 \\
\hline
\end{tabular}




\subsection{Prevalencia de depresión y su relación con las variables}

De la población estudiada, el 32.63\% tenía algún grado de depresión según los criterios utilizados (ver tabla 10), presentando un $7.37 \%$ criterios de depresión grave. La figura 3 muestra los niveles de depresión según sexo.

Tabla 10: Prevalencia de depresión.

\begin{tabular}{|c|c|c|c|c|c|c|c|}
\hline \multirow{3}{*}{$\begin{array}{c}\text { Sintomatología } \\
\text { depresiva }\end{array}$} & \multicolumn{6}{|c|}{ Sexo } & \multirow{3}{*}{ P-value } \\
\hline & \multicolumn{2}{|c|}{ Hombres } & \multicolumn{2}{|c|}{ Mujeres } & \multicolumn{2}{|c|}{ Total } & \\
\hline & $N$ & $\%$ & $N$ & $\%$ & $N$ & $\%$ & \\
\hline Sin depresión & 42 & 75.00 & 22 & 56.41 & 64 & 67.37 & 0.057 \\
\hline Depresión & 14 & 25.00 & 17 & 43.59 & 31 & 32.63 & \\
\hline
\end{tabular}

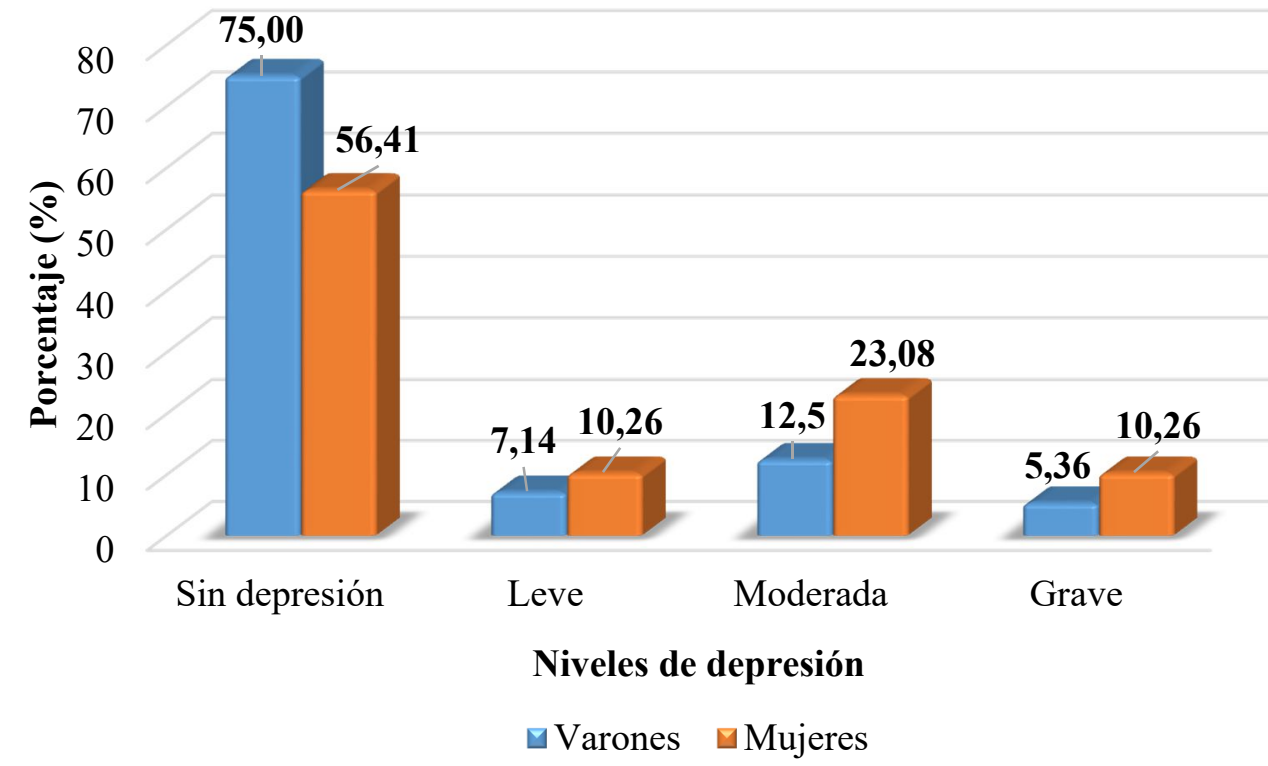

Figura 3: Niveles de depresión según sexo.

Diagrama de barras en el que se muestra la distribución de los diversos niveles de depresión según el sexo del paciente. Se puede observar que las mujeres tienen una mayor prevalencia de la depresión en todos los niveles. 
De los pacientes que presentaban depresión el $12.63 \%$ ya estaba recibiendo tratamiento antidepresivo durante el estudio y el $17.89 \%$ estaba con tratamiento ansiolítico no existiendo diferencia significativa por sexos (ver tabla 11).

Tabla 11: Tratamiento antidepresivo y ansiolítico en BDI-II alterado.

\begin{tabular}{|c|c|c|c|c|c|c|c|}
\hline \multirow{3}{*}{ Tipo de tratamiento } & \multicolumn{6}{|c|}{ Sexo } & \multirow{3}{*}{ P-value } \\
\hline & \multicolumn{2}{|c|}{ Hombres } & \multicolumn{2}{|c|}{ Mujeres } & \multicolumn{2}{|c|}{ Total } & \\
\hline & $N$ & $\%$ & $N$ & $\%$ & $N$ & $\%$ & \\
\hline \multicolumn{8}{|c|}{ Tratamiento antidepresivo } \\
\hline No & 52 & 92.86 & 31 & 79.49 & 83 & 87.37 & 0.054 \\
\hline $\mathrm{Si}$ & 4 & 7.14 & 8 & 20.51 & 12 & 12.63 & \\
\hline \multicolumn{8}{|l|}{ Tratamiento ansiolítico } \\
\hline No & 47 & 83.93 & 31 & 79.49 & 78 & 82.11 & 0.579 \\
\hline $\mathrm{Si}$ & 9 & 16.07 & 8 & 20.51 & 17 & 17.89 & \\
\hline
\end{tabular}

Respecto a la relación de la depresión con las características generales, se puede observar que el ser mujer, presentar un menor nivel educativo y no estar casado(a) aumentaban la presencia de depresión, aunque estas asociaciones no fueron estadísticamente significativas. Lo mismo sucede al analizar el estado cognitivo ya que aunque se encontró que aquellos que presentaban mayor deterioro cognitivo según la puntuación del MEC30 presentaban un OR mayor, los resultados no fueron estadísticamente significativos. En la tabla 12 se analiza la relación de la depresión con estas variables.

Tampoco se encontró asociación con el antecedente familiar de EP, la edad de inicio, el tiempo de evolución de la EP ni su estadío. Cabe destacar que, aunque no se encontró una diferencia estadísticamente significativa, aquellos que tenían más de 10 años de diagnóstico tenían casi 3 veces más riesgo de presentar síntomas depresivos que aquellos que fueron diagnosticados hace menos de 3 años (ver tabla 13). 
Tabla 12: Relación de la depresión y variables sociodemográficas y estado cognitivo.

\begin{tabular}{lcccc}
\hline \hline Variables & Odds Ratio & DS & p valor & IC:95\% \\
\hline \hline Sexo & & & & \\
$\quad$ Masculino & 1.00 & & & \\
$\quad$ Femenino & 2.32 & 1.04 & 0.060 & $0.97-5.56$ \\
Nivel Educativo & & & & \\
$\quad$ Superior & 1.00 & & & \\
$\quad$ Ninguna & 5.40 & 5.63 & 0.106 & $0.70-41.75$ \\
$\quad$ Primaria & 2.40 & 1.44 & 0.144 & $0.74-7.78$ \\
$\quad$ Secundaria & 1.26 & 0.84 & 0.730 & $0.34-4.68$ \\
Estado Civil & & & & \\
$\quad$ Casados & 1.00 & & & \\
$\quad$ No casados & 1.89 & 0.89 & 0.172 & $0.76-4.74$ \\
Sector de Trabajo & & & & \\
$\quad$ Ama de casa & 1.00 & & & \\
$\quad$ Primario & 0.35 & 0.32 & 0.252 & $0.58-2.12$ \\
$\quad$ Industrial & 0.85 & 0.57 & 0.804 & $0.23-3.17$ \\
$\quad$ Servicios & 0.76 & 0.44 & 0.635 & $0.25-2.36$ \\
Estado cognitivo & & & & \\
$\quad \begin{array}{l}\text { Normal } \\
\text { Sospecha de patología }\end{array}$ & 1.75 & 1.40 & 0.485 & $0.36-8.42$ \\
$\quad$ Deterioro & 2.33 & 1.75 & 0.257 & $0.54-10.11$ \\
\hline \hline
\end{tabular}


Tabla 13: Relación de la depresión y características de la Enfermedad de Parkinson

\begin{tabular}{lcccc}
\hline \multicolumn{1}{c}{ Variables } & Odds Ratio & DS & p valor & IC:95\% \\
\hline \hline $\begin{array}{l}\text { Antecedente familiar } \\
\quad \text { No }\end{array}$ & 1.00 & & & \\
$\quad$ Sí & 0.52 & 0.26 & 0.192 & $0.19-1.39$ \\
Edad de inicio (años) & & & & \\
$\quad \geq 50$ & 1.00 & & & \\
$\quad<50$ & 0.87 & 0.63 & 0.851 & $0.21-3.63$ \\
Tiempo de evolución (años) & & & & \\
$\quad<3$ & 1.00 & & & \\
$\geq 3$ y $<6$ & 1.31 & 0.91 & 0.700 & $0.34-5.09$ \\
$\geq 6$ y $\leq 10$ & 0.70 & 0.48 & 0.607 & $0.18-2.72$ \\
$\quad>10$ & 3.31 & 2.19 & 0.071 & $0.90-12.13$ \\
Estadíos de Hoehn y Yahr & & & & \\
0 & 1.00 & & & \\
1.0 & 0.50 & 0.66 & 0.600 & $0.037-6.68$ \\
1.5 & 2.50 & 3.49 & 0.512 & $0.16-38.60$ \\
2.0 & 0.33 & 0.54 & 0.501 & $0.01-8.18$ \\
2.5 & 0.80 & 1.09 & 0.870 & $0.06-11.50$ \\
3.0 & 1.43 & 1.85 & 0.783 & $0.11-18.00$ \\
4.0 & 1.25 & 1.69 & 0.869 & $0.09-17.65$ \\
\hline \hline
\end{tabular}


Se hallaron resultados similares en la asociación con la cantidad de tratamientos recibidos para el Parkinson ya que aunque no se encontró una relación estadísticamente significativa, se evidenció una disminución del riesgo de depresión cuando aumentaban el número de grupos terapéuticos que consumían (ver tabla 14).

Tabla n 14: Relación de la depresión y tratamientos antiparkinsonianos.

\begin{tabular}{ccccc}
\hline \hline Variables & Odds Ratio & DS & p valor & IC:95\% \\
\hline \hline Tratamiento total & & & & \\
0 & 1.00 & & & \\
1 & 0.88 & 0.99 & 0.906 & $0.096-7.95$ \\
2 & 0.50 & 0.54 & 0.521 & $0.06-4.15$ \\
3 & 0.42 & 0.45 & 0.413 & $0.05-3.38$ \\
$\geq 4$ & 0.25 & 0.30 & 0.244 & $0.024-2.58$ \\
Tratamiento oral & & & & \\
0 & 1.00 & & & \\
1 & 0.89 & 0.99 & 0.916 & $0.10-7.86$ \\
2 & 0.52 & 0.56 & 0.545 & $0.65-4.24$ \\
3 & 0.32 & 0.35 & 0.293 & $0.38-2.70$ \\
$\geq 4$ & 0.30 & 0.36 & 0.315 & $0.29-3.13$ \\
Terapia avanzada & & & & \\
Ausente & 1.00 & & & \\
Presente & 1.20 & 0.81 & 0.779 & $0.33-4.48$ \\
\hline \hline
\end{tabular}

Se analizó la relación de la depresión con otras enfermedades médicas (ver tabla 15), no encontrándose relación estadísticamente significativa con ninguna de las patologías estudiadas. Lo mismo se encontró en aquellos pacientes que ya habían sido diagnosticados previamente de alguna patología de salud mental (ver tabla 16). 
Tabla 15: Relación de la depresión y otras enfermedades médicas.

\begin{tabular}{|c|c|c|c|c|}
\hline Enfermedades médicas & Odds Ratio & $D S$ & p valor & $I C: 95 \%$ \\
\hline \multicolumn{5}{|l|}{ Cardiovascular } \\
\hline Ausente & 1.00 & & & \\
\hline Presente & 1.28 & 0.57 & 0.571 & $0.54-3.06$ \\
\hline \multicolumn{5}{|l|}{ Pulmonar } \\
\hline Ausente & 1.00 & & & \\
\hline Presente & 1.72 & 1.22 & 0.446 & $0.43-6.91$ \\
\hline \multicolumn{5}{|l|}{ Digestivo } \\
\hline Ausente & 1.00 & & & \\
\hline Presente & 2.14 & 2.19 & 0.459 & $0.29-15.94$ \\
\hline \multicolumn{5}{|l|}{ Oncológica } \\
\hline Ausente & 1.00 & & & \\
\hline Presente & 0.68 & 0.80 & 0.741 & $0.68-6.79$ \\
\hline \multicolumn{5}{|l|}{ Otras } \\
\hline Ausente & 1.00 & & & \\
\hline Presente & 1.56 & 0.79 & 0.382 & $0.58-4.21$ \\
\hline
\end{tabular}

Tabla 16: Relación entre depresión y antecedente de patología de Salud Mental.

\begin{tabular}{ccccc}
\hline \hline $\begin{array}{l}\text { Enfermedades } \\
\text { psiquiátricas }\end{array}$ & Odds Ratio & DS & p valor & IC:95\% \\
\hline $\begin{array}{c}\text { Depresión } \\
\text { Ausente } \\
\quad \text { Presente }\end{array}$ & 1.00 & & & \\
$\begin{array}{c}\text { Ansiedad } \\
\text { Ausente }\end{array}$ & 2.21 & 1.16 & 0.131 & $0.79-6.18$ \\
$\quad$ Presente & 1.00 & & & \\
Otra $\quad$ Ausente & 2.88 & 2.05 & 0.136 & $0.72-11.62$ \\
$\quad$ Presente & 1.00 & & & \\
\hline \hline
\end{tabular}


Para el análisis de la relación de la depresión con la calidad de vida, se estratificaron las diversas dimensiones del PDQ-39 en cuartiles (movilidad, actividades de la vida diaria, bienestar emocional, estado cognitivo y dolor) o terciles (estigmatización, apoyo social y comunicación) de acuerdo a la distribución de los resultados obtenidos. Tras el análisis de regresión se encontró que aquellos que tenían peores puntuaciones en las dimensiones de movilidad tenían 6.11 veces más riesgo de padecer depresión (OR: 6.11, p = 0.006); los que presentaban puntuaciones peores para realizar las actividades de la vida diaria tenían 7.09 veces más riesgo de padecer depresión (OR: 7.09, p =0.009); los que tenían peor bienestar emocional tenían 56.67 veces más riesgo de padecer depresión (OR: 56.67, $\mathrm{p}=<0.001)$; los que tenían mayores puntuaciones de estigmatización tenían 3.06 veces más riesgo de padecer depresión (OR: 3.06, $\mathrm{p}=0.018$ ), aquellos con mayores puntuaciones en la dimensión de estado cognitivo tenía 12.44 veces más riesgo de padecer depresión (OR: 12.44, $\mathrm{p}=0,028)$ y los que tenían peores puntuaciones en comunicación tenían 6.4 veces más riesgo de padecer depresión $(\mathrm{OR}: 6.40, \mathrm{p}=0.002)$. No se encontró relación de depresión con las dimensiones de apoyo social y de dolor (ver tabla 17).

Se calculó también la relación del PDSI con la depresión, dividiendo los valores obtenidos en cuartiles. Se observó que a mayores puntuaciones de PDSI (menor calidad de vida) aumentaba el riesgo de depresión, llegando a 83.6 veces más riesgo en aquellos que estaban en el cuarto cuartil (OR: 83.60, $\mathrm{p}=<0.001)$ (ver tabla 18). 
Tabla 17: Relación de la depresión y la calidad de vida según el PDQ-39.

\begin{tabular}{|c|c|c|c|c|}
\hline Calidad de Vida & Odds Ratio & $D S$ & p valor & $I C: 95 \%$ \\
\hline \multicolumn{5}{|c|}{ Movilidad (cuartiles) } \\
\hline 1 & 1.00 & & & \\
\hline 2 & 0.24 & 0.28 & 0.216 & $0.02-2.32$ \\
\hline 3 & 3.45 & 2.49 & 0.085 & $0.84-14.17$ \\
\hline 4 & 6.11 & 4.00 & 0.006 & $1.69-22.07$ \\
\hline \multicolumn{5}{|c|}{ Actividades de la vida diaria } \\
\hline 1 & 1.00 & & & \\
\hline 2 & 0.98 & 0.80 & 0.985 & $0.20-4.82$ \\
\hline 3 & 1.20 & 0.98 & 0.820 & $0.24-5.96$ \\
\hline 4 & 7.09 & 5.30 & 0.009 & $1.64-30.81$ \\
\hline \multicolumn{5}{|c|}{ Bienestar emocional } \\
\hline 1 & 1.00 & & & \\
\hline 2 & 3.86 & 4.41 & 0.237 & $0.41-36.23$ \\
\hline 3 & 4.47 & 5.12 & 0.191 & $0.47-42.21$ \\
\hline 4 & 56.67 & 64.00 & $<0.001$ & $6.19-518.39$ \\
\hline \multicolumn{5}{|l|}{ Estigmatización } \\
\hline 1 & 1.00 & & & \\
\hline 2 & 1.85 & 1.73 & 0.511 & $0.30-11.60$ \\
\hline 3 & 3.06 & 1.44 & 0.018 & $1.21-7.71$ \\
\hline \multicolumn{5}{|l|}{ Apoyo social } \\
\hline 1 & 1.00 & & & \\
\hline 2 & 1.55 & 0.83 & 0.416 & $0.54-4.42$ \\
\hline 3 & 1.83 & 0.97 & 0.252 & $0.65-5.17$ \\
\hline \multicolumn{5}{|l|}{ Estado cognitivo } \\
\hline 1 & 1.00 & & & \\
\hline 2 & 0.88 & 1.04 & 0.911 & $0.84-9.07$ \\
\hline 3 & 4.38 & 5.00 & 0.196 & $0.47-41.07$ \\
\hline 4 & 12.44 & 14.28 & 0.028 & $1.31-117.93$ \\
\hline \multicolumn{5}{|l|}{ Comunicación } \\
\hline 1 & 1.00 & & & \\
\hline 2 & 2.55 & 1.57 & 0.130 & $0.76-8.54$ \\
\hline 3 & 6.40 & 3.89 & 0.002 & $1.94-21.07$ \\
\hline \multicolumn{5}{|l|}{ Dolor } \\
\hline 1 & 1.00 & & & \\
\hline 2 & 0.23 & 0.21 & 0.111 & $0.38-1.40$ \\
\hline 3 & 1.17 & 0.82 & 0.818 & $0.30-4.58$ \\
\hline 4 & 1.85 & 1.18 & 0.333 & $0.53-6.46$ \\
\hline
\end{tabular}


Tabla 18: Relación del PDSI y depresión.

\begin{tabular}{ccccc}
\hline \hline $\begin{array}{c}\text { PDSI } \\
\text { (cuartiles) }\end{array}$ & Odds Ratio & DS & p valor & IC:95\% \\
\hline \hline 1 & 1.00 & & & \\
2 & 4.40 & 5.10 & 0.201 & $0.45-42.74$ \\
3 & 9.06 & 10.11 & 0.048 & $1.02-80.84$ \\
4 & 83.60 & 95.25 & $<0.001$ & $8.96-779.85$ \\
\hline \hline
\end{tabular}

En el análisis de regresión logística múltiple se encontró un aumento del riesgo de depresión en 35.80 veces en aquellos pacientes con más de 10 años de evolución de la enfermedad (OR: 35.80, $\mathrm{p}=0.040)$ así como un aumento progresivo de dicho riesgo al presentar una peor calidad de vida relacionada con la salud medida con el PDSI (cuartil 4 del PDSI: OR: 2521.00, $\mathrm{p}=<0.001$ ). Por otro lado, se evidenció un mayor OR a menor escolarización y en aquellos que no estaban casados, aunque en estos casos no se alcanzó el valor de significancia necesaria (ver tabla 19). 
Tabla 19: Análisis de regresión logística múltiple de la depresión y otras variables.

\begin{tabular}{|c|c|c|c|c|}
\hline Variables & Odds Ratio & $D S$ & p valor & $I C: 95 \%$ \\
\hline \multicolumn{5}{|l|}{ Sexo } \\
\hline Masculino & 1.00 & & & \\
\hline Femenino & 0.53 & 0.58 & 0.565 & $0.06-4.59$ \\
\hline \multicolumn{5}{|l|}{ Nivel Educativo } \\
\hline Superior & 1.00 & & & \\
\hline Ninguna & 5.73 & 14.72 & 0.497 & $0.04-880.47$ \\
\hline Primaria & 3.49 & 4.71 & 0.354 & $0.25-49.15$ \\
\hline Secundaria & 0.25 & 0.33 & 0.286 & $0.02-3.17$ \\
\hline \multicolumn{5}{|l|}{ Estado Civil } \\
\hline Casados & 1.00 & & & \\
\hline No casados & 6.64 & 8.13 & 0.122 & $0.60-73.25$ \\
\hline \multicolumn{5}{|l|}{ Tiempo de evolución (años) } \\
\hline$<3$ & 1.00 & & & \\
\hline $3-5$ & 0.27 & 0.40 & 0.375 & $0.01-4.82$ \\
\hline $6-10$ & 0.87 & 1.36 & 0.928 & $0.04-18.83$ \\
\hline$>10$ & 35.80 & 62.31 & 0.040 & $1.18-1084.93$ \\
\hline \multicolumn{5}{|l|}{ Estadíos de Hoehn y Yahr } \\
\hline 0 & 1.00 & & & \\
\hline 1.0 & 1.89 & 4.47 & 0.789 & $0.02-196.22$ \\
\hline 1.5 & 2.34 & 5.96 & 0.739 & $0.02-346.43$ \\
\hline 2.0 & 2.77 & 7.75 & 0.716 & $0.01-667.55$ \\
\hline 2.5 & 1.65 & 4.06 & 0.838 & $0.01-203.93$ \\
\hline 3.0 & 0.64 & 1.44 & 0.842 & $0.01-53.10$ \\
\hline 4.0 & 0.02 & 0.04 & 0.105 & $0.00-2.36$ \\
\hline \multicolumn{5}{|l|}{ Tratamiento oral + avanzado } \\
\hline 0 & 1.00 & & & \\
\hline 1 & 6.27 & 12.25 & 0.347 & $0.14-287.91$ \\
\hline 2 & 3.40 & 6.61 & 0.528 & $0.08-152.96$ \\
\hline 3 & 1.43 & 2.88 & 0.858 & $0.03-73.32$ \\
\hline$\geq 4$ & 0.99 & 2.15 & 0.996 & $0.01-70.47$ \\
\hline \multicolumn{5}{|l|}{ Estado cognitivo } \\
\hline Normal & 1.00 & & & \\
\hline Sospecha de patología & 7.97 & 14.55 & 0.255 & $0.22-285.40$ \\
\hline Deterioro & 2.60 & 3.49 & 0.477 & $0.19-36.08$ \\
\hline \multicolumn{5}{|l|}{ PDSI } \\
\hline 1 & 1.00 & & & \\
\hline 2 & 17.13 & 29.11 & 0.094 & $0.61-478.63$ \\
\hline 3 & 59.88 & 94.48 & 0.009 & $2.72-1319.27$ \\
\hline 4 & 2521.00 & 5188.61 & $<0.001$ & $44.63-142390.5$ \\
\hline
\end{tabular}




\subsection{Prevalencia de ansiedad y su relación con las variables}

La puntuación media del STAI-R en varones fue de $24.86( \pm 8.83)$ y $27.69( \pm 10.05)$ en mujeres que se corresponden con los centiles 74 y 63 respectivamente, no existiendo diferencias significativas por sexos. Además, se analizó de forma puntual en STAI-E para ver el nivel de ansiedad al momento de la entrevista (ver tabla 20).

Tabla 20: Puntuación directa y centil del STAI según sexo.

\begin{tabular}{|c|c|c|c|c|c|c|c|c|c|}
\hline \multirow{3}{*}{$S T A I$} & \multicolumn{8}{|c|}{ Sexo } & \multirow{3}{*}{ p-value } \\
\hline & \multicolumn{3}{|c|}{ Hombres } & \multicolumn{3}{|c|}{ Mujeres } & \multicolumn{2}{|c|}{ Total } & \\
\hline & Media & $D E$ & Centil & Media & $D E$ & Centil & Media & $D E$ & \\
\hline STAI-E & 23.86 & 8.89 & 64 & 21.49 & 10.99 & 52 & 22.88 & 9.82 & 0.132 \\
\hline STAI-R & 24.86 & 8.83 & 74 & 27.69 & 10.05 & 63 & 26.02 & 9.40 & 0.240 \\
\hline
\end{tabular}

Según los criterios de ansiedad utilizados, al convertir las puntuaciones en centiles y considerar ansiedad como centil $>50$, el 58.95\% de los participantes presentaba un estado de ansiedad elevado al momento del estudio; mientras que el $68.42 \%$ presentaba rasgos de ansiedad elevados, siendo ambas situaciones más frecuentes en varones (ver figuras 4 y 5$)$.

Centrándose en la ansiedad como rasgo, que es en la que se basa el presente estudio, en la tabla 21 se puede observar la distribución de la población según sus niveles de ansiedad (STAI-R). Se encontró que el $42.11 \%$ de la población estudiada tenía niveles muy altos de ansiedad (centil >75) siendo más frecuente en los varones. En la figura 6 se puede observar mejor la distribución de la ansiedad en la población estudiada. 


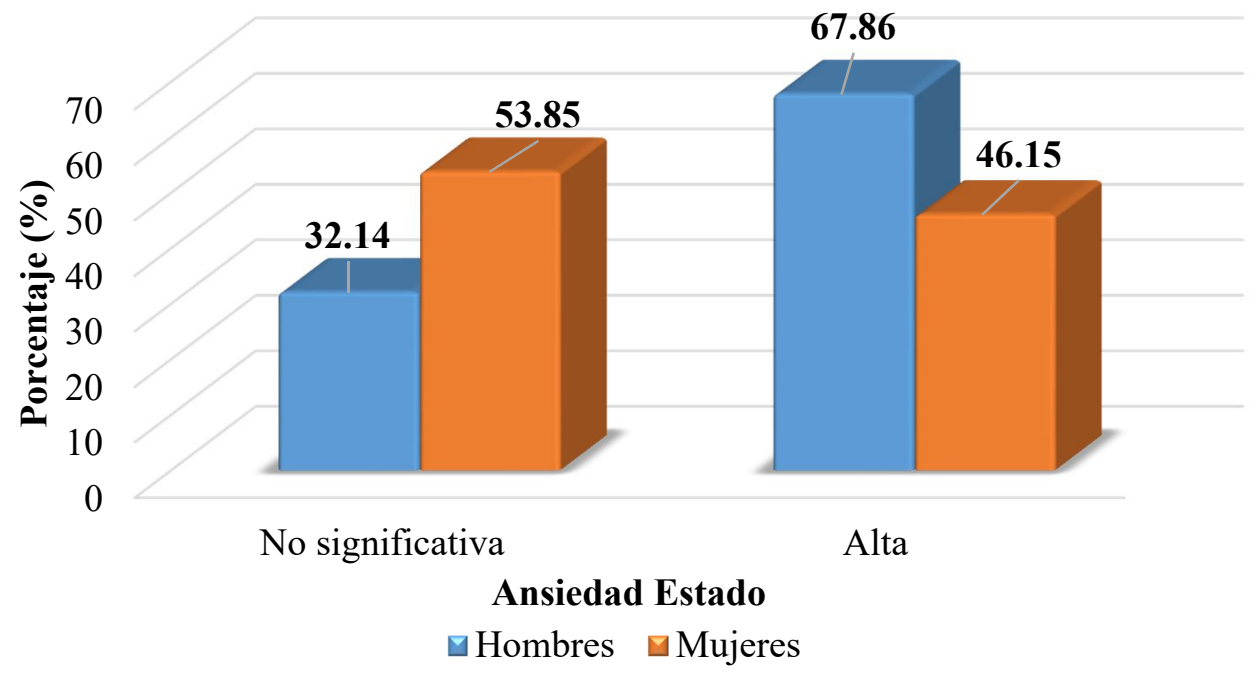

Figura 4: Presencia de Ansiedad Estado según sexo.

Diagrama de barras que muestra la distribución de la ansiedad estado (medido por el STAI-E) según el sexo observándose que los niveles elevados son más frecuentes en varones.

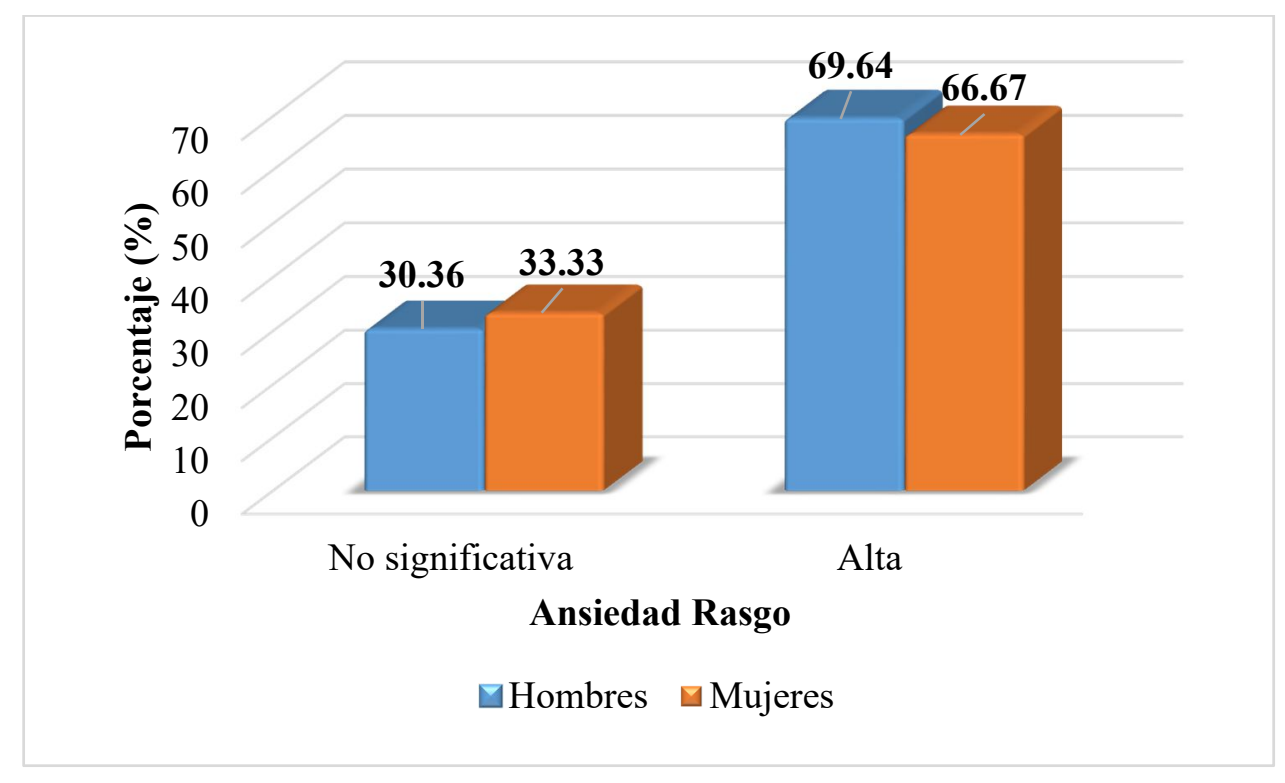

Figura 5: Presencia de Ansiedad Rasgo según sexo.

Diagrama de barras que muestra la distribución de la ansiedad rasgo (medido por el STAI-R) según el sexo observándose que los niveles elevados son discretamente más frecuentes en varones (no significativo). 
Tabla $n^{0}$ 21: Estratificación de los niveles de ansiedad.

\begin{tabular}{|c|c|c|c|c|c|c|c|}
\hline \multirow{3}{*}{$\begin{array}{c}\text { Sintomatología } \\
\text { Ansiosa }(S T A I-R)\end{array}$} & \multicolumn{6}{|c|}{ Sexo } & \multirow{3}{*}{ P-value } \\
\hline & \multicolumn{2}{|c|}{ Hombres } & \multicolumn{2}{|c|}{ Mujeres } & \multicolumn{2}{|c|}{ Total } & \\
\hline & $N$ & $\%$ & $N$ & $\%$ & $N$ & $\%$ & \\
\hline Baja & 9 & 16,07 & 6 & 15,28 & 15 & 15,79 & \\
\hline Media-baja & 8 & 14,29 & 7 & 17,95 & 15 & 15,79 & 0,074 \\
\hline Media-alta & 10 & 17,86 & 15 & 38,46 & 25 & 26,31 & \\
\hline Muy alta & 29 & 51,78 & 11 & 28,21 & 40 & 42,11 & \\
\hline
\end{tabular}

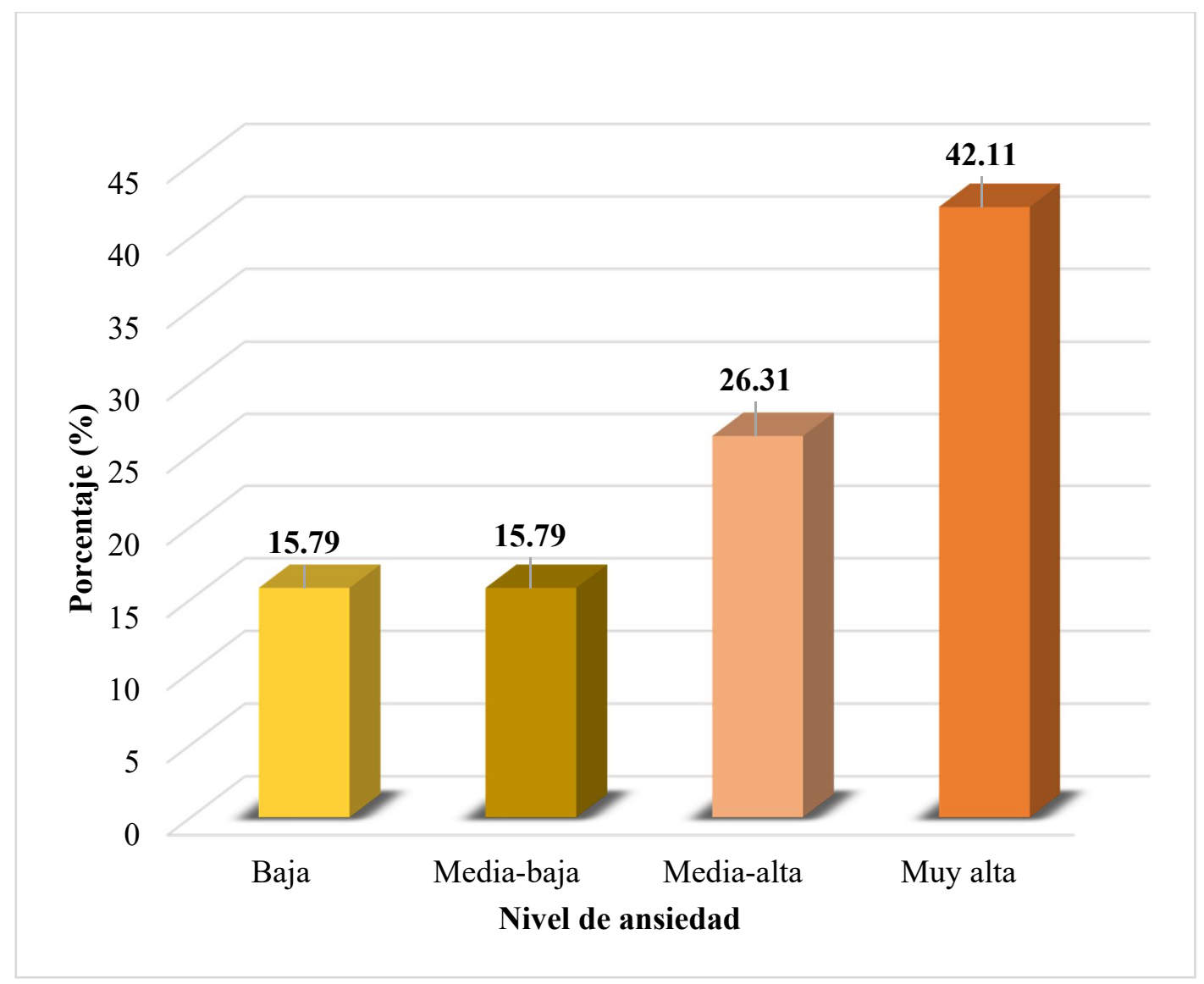

Figura 6: Distribución de la ansiedad por intensidad.

Diagrama de barras que muestra la distribución de los niveles de ansiedad (medido por el STAI-R). Se puede observar la mayor frecuencia de los niveles muy altos de ansiedad. 
De los pacientes que presentaban ansiedad (STAI-R $>50$ ) el $28.42 \%$ de los que presentaba centiles elevados ya se encontraban tomando ansiolíticos y el $18.95 \%$ antidepresivos. El consumo de estos últimos fue más frecuente en las mujeres presentando una diferencia estadísticamente significativa $(p=0.003)$ (ver tabla 22).

Tabla 22: Tratamiento ansiolítico y antidepresivo en pacientes con ansiedad.

\begin{tabular}{|c|c|c|c|c|c|c|c|}
\hline \multirow{3}{*}{ Tipo de tratamiento } & \multicolumn{6}{|c|}{ Sexo } & \multirow{3}{*}{ P-value } \\
\hline & \multicolumn{2}{|c|}{ Hombres } & \multicolumn{2}{|c|}{ Mujeres } & \multicolumn{2}{|c|}{ Total } & \\
\hline & $N$ & $\%$ & $N$ & $\%$ & $N$ & $\%$ & \\
\hline \multicolumn{8}{|l|}{ Tratamiento ansiolítico } \\
\hline No & 40 & 71.43 & 28 & 71.79 & 68 & 71.58 & 0.969 \\
\hline Sí & 16 & 28.57 & 11 & 28.21 & 27 & 28.42 & \\
\hline \multicolumn{8}{|c|}{ Tratamiento antidepresivo } \\
\hline No & 51 & 91.07 & 26 & 66.67 & 77 & 81.05 & 0.003 \\
\hline Sí & 5 & 8.93 & 13 & 33.33 & 18 & 18.95 & \\
\hline
\end{tabular}

Por otro lado, se encontró que el 31.58\% de los pacientes estudiados tenían valores de depresión y ansiedad (STAI-R) elevados, presentándose en mayor proporción en las mujeres aunque sin significación estadística (ver tabla 23).

Tabla 23: Coexistencia de depresión y ansiedad según sexo.

\begin{tabular}{|c|c|c|c|c|c|c|c|}
\hline \multirow{3}{*}{$\begin{array}{c}\text { Ansiedad y Depresión } \\
\text { (STAI-R + Beck-II) }\end{array}$} & \multicolumn{6}{|c|}{ Sexo } & \multirow{3}{*}{ P-value } \\
\hline & \multicolumn{2}{|c|}{ Hombres } & \multicolumn{2}{|c|}{ Mujeres } & \multicolumn{2}{|c|}{ Total } & \\
\hline & $N$ & $\%$ & $N$ & $\%$ & $N$ & $\%$ & \\
\hline No & 42 & 75.00 & 23 & 58.97 & 65 & 68.42 & 0.098 \\
\hline Sí & 14 & 25.00 & 16 & 41.03 & 30 & 31.58 & \\
\hline
\end{tabular}


Cuando se consideró sólo a los pacientes con depresión se observa que el 96.77\% de los pacientes deprimidos presentaba también ansiedad.

Respecto a la relación de la ansiedad con las características generales de la población, no se encontraron diferencias estadísticamente significativas con las variables sociodemográficas (sexo, nivel educativo, estado civil y sector de trabajo). Al analizar el nivel cognitivo de la población, si bien se aprecia cierto aumento del OR en aquellos pacientes en los que se sospecha deterioro cognitivo y presencia de ansiedad, este valor no llega a ser significativo (ver tabla 24 ).

Tabla 24: Relación de la ansiedad y las diversas variables.

\begin{tabular}{lcccc}
\hline \multicolumn{1}{c}{ Variables } & Odds Ratio & DS & p valor & IC:95\% \\
\hline \hline Sexo & & & & \\
Masculino & 1.00 & & & \\
$\quad$ Femenino & 0.87 & 0.39 & 0.759 & $0.36-2.09$ \\
Nivel Educativo & & & & \\
$\quad$ Superior & 1.00 & & & \\
$\quad$ Ninguna & 2.57 & 3.08 & 0.430 & $0.25-26.85$ \\
$\quad$ Primaria & 1.34 & 0.73 & 0.596 & $0.46-3.88$ \\
$\quad$ Secundaria & 1.84 & 1.13 & 0.321 & $0.55-6.10$ \\
Estado Civil & & & & \\
$\quad$ Casados & 1.00 & & & \\
$\quad$ No casados & 0.61 & 0.29 & 0.298 & $0.24-1.54$ \\
Sector de Trabajo & & & & \\
Ama de casa & 1.00 & & & \\
$\quad$ Primario & 1.70 & 1.41 & 0.530 & $0.33-8.67$ \\
Industrial & 1.18 & 0.80 & 0.804 & $0.32-4.44$ \\
Servicios & 1.62 & 0.94 & 0.411 & $0.51-5.07$ \\
Estado Cognitivo & & & & \\
$\quad$ Normal & 1.00 & & & \\
$\quad$ Sospecha de patología & 3.06 & 3.38 & 0.312 & $0.35-26.69$ \\
Deterioro & 1.53 & 1.30 & 0.618 & $0.29-8.09$ \\
\hline \hline
\end{tabular}


Se buscó si existía relación entre algunas características de la EP, tales como la presencia de historia familiar de dicha patología, la edad de inicio de la enfermedad, su tiempo de evolución y el estadío en el que se encontraban los pacientes al momento del estudio, sin encontrarse ninguna asociación estadísticamente significativa (ver tabla 25).

Tabla 25: Relación de la ansiedad y características de la Enfermedad de Parkinson

\begin{tabular}{lcccc}
\hline \hline \multicolumn{1}{c}{ Variables } & Odds Ratio & DS & p valor & IC:95\% \\
\hline \hline $\begin{array}{l}\text { Antecedente familiar } \\
\quad \text { No }\end{array}$ & 1.00 & & & \\
$\quad$ Sí & 0.48 & 0.23 & 0.125 & $0.19-1.22$ \\
Edad de inicio (años) & & & & \\
$\quad \geq 50$ & 1.00 & & & \\
$\quad<50$ & 1.08 & 0.79 & 0.910 & $0.26-4.53$ \\
Tiempo de evolución (años) & & & & \\
$\quad<3$ & 1.00 & & & \\
$\quad \geq 3$ y $<6$ & 2.63 & 1.91 & 0.186 & $0.63-10.96$ \\
$\quad \geq 6$ y $\leq 10$ & 0.67 & 0.40 & 0.499 & $0.21-2.16$ \\
$\quad>10$ & 2.22 & 1.53 & 0.250 & $0.57-8.60$ \\
Estadíos de Hoehn y Yahr & & & & \\
0 & 1.00 & & & \\
1.0 & 0.75 & 0.97 & 0.824 & $0.06-9.42$ \\
1.5 & 1.00 & 1.41 & 1.000 & $0.06-15.99$ \\
2.0 & 0.67 & 0.96 & 0.779 & $0.04-11.29$ \\
2.5 & 1.25 & 1.70 & 0.870 & $0.09-17.98$ \\
3.0 & 1.50 & 1.97 & 0.757 & $0.11-19.64$ \\
4.0 & 1.67 & 2.32 & 0.713 & $0.11-25.43$ \\
& & & & \\
\hline \hline
\end{tabular}


Se analizó la relación de la ansiedad con los tratamientos recibidos para el Parkinson no encontrándose una relación estadísticamente significativa. Se encontró cierta disminución del OR cuando los pacientes recibían mayor cantidad de tratamientos aunque ésta no llegaba a valores estadísticamente significativos (tabla 26). Cabe señalar que en las variables tratamiento total (oral + avanzado) y tratamiento oral, se suprimieron del análisis aquellas personas que no recibían ningún tipo de tratamiento ya que la cantidad escasa de pacientes en dichos grupos limitaba su análisis.

Tabla 26: Relación de la ansiedad y tratamientos antiparkinsonianos.

\begin{tabular}{lcccc}
\hline \hline Variables & Odds Ratio & DS & p valor & IC:95\% \\
\hline \hline Tratamiento total & & & & \\
1 & 1.00 & & & \\
2 & 0.50 & 0.38 & 0.364 & $0.11-2.23$ \\
3 & 0.52 & 0.39 & 0.382 & $0.12-2.24$ \\
$\geq 4$ & 0.29 & 0.24 & 0.130 & $0.06-1.44$ \\
Tratamiento oral & & & & \\
1 & 1.00 & & & \\
2 & 0.41 & 0.30 & 0.225 & $0.097-1.73$ \\
3 & 0.41 & 0.30 & 0.229 & $0.09-1.76$ \\
$\geq 4$ & 0.25 & 0.21 & 0.101 & $0.048-1.31$ \\
Terapia avanzada & & & & \\
Ausente & 1.00 & & & \\
Presente & 1.26 & 0.90 & 0.744 & $0.31-5.14$ \\
\hline \hline
\end{tabular}

Al analizar la relación de la ansiedad con otras enfermedades médicas, no se encontró relación estadísticamente significativa con ninguna de las enfermedades médicas estudiadas; sin embargo, aunque no se aprecia relación significativa cabe destacar un aumento discreto del OR en las pacientes con enfermedades pulmonares (ver tabla 27). 
Tabla 27: Relación de la ansiedad y otras enfermedades médicas.

\begin{tabular}{|c|c|c|c|c|}
\hline Enfermedades médicas & Odds Ratio & $D S$ & p valor & IC:95\% \\
\hline \multicolumn{5}{|l|}{ Cardiovascular } \\
\hline Ausente & 1.00 & & & \\
\hline Presente & 1.61 & 0.74 & 0.301 & $0.65-3.97$ \\
\hline \multicolumn{5}{|l|}{ Pulmonar } \\
\hline Ausente & 1.00 & & & \\
\hline Presente & 3.93 & 4.27 & 0.207 & $0.47-32.99$ \\
\hline \multicolumn{5}{|l|}{ Digestivo } \\
\hline Ausente & 1.00 & & & \\
\hline Presente & 0.44 & 0.46 & 0.429 & $0.06-3.32$ \\
\hline \multicolumn{5}{|l|}{ Oncológica } \\
\hline Ausente & 1.00 & & & \\
\hline Presente & 0.44 & 0.46 & 0.429 & $0.06-3.32$ \\
\hline \multicolumn{5}{|l|}{ Otras } \\
\hline Ausente & 1.00 & & & \\
\hline Presente & 0.88 & 0.44 & 0.797 & $0.33-2.32$ \\
\hline
\end{tabular}

Al analizar la relación de la ansiedad con las patologías de salud mental diagnosticadas previamente, tampoco se encontró una relación estadísticamente significativa. Cabe destacar que sin contar los diagnósticos previos de depresión y ansiedad, los trastornos del sueño eran los más prevalentes (ver tabla 28). 
Tabla 28: Relación entre ansiedad y antecedente de patología de Salud Mental.

\begin{tabular}{ccccc}
\hline \hline $\begin{array}{c}\text { Enfermedades } \\
\text { psiquiátricas }\end{array}$ & Odds Ratio & DS & p valor & IC:95\% \\
\hline \hline $\begin{array}{c}\text { Depresión } \\
\text { Ausente } \\
\quad \text { Presente }\end{array}$ & 1.00 & & & \\
Ansiedad & 1.37 & 0.79 & 0.582 & $0.44-4.24$ \\
$\quad \begin{array}{l}\text { Ausente } \\
\text { Presente }\end{array}$ & 1.00 & & & \\
Otra & 1.69 & 1.40 & 0.529 & $0.33-8.67$ \\
$\quad \begin{array}{l}\text { Ausente } \\
\text { Presente }\end{array}$ & 1.00 & & & \\
\hline \hline
\end{tabular}

Para el análisis de la relación de la ansiedad con la calidad de vida, se estratificaron las diversas dimensiones del PDQ-39 en cuartiles (movilidad, actividades de la vida diaria, bienestar emocional, estado cognitivo y dolor) o terciles (estigmatización, apoyo social y comunicación) de acuerdo a la distribución de los resultados obtenidos. Tras el análisis de regresión se encontró que, en casi todas las dimensiones, aquellos pacientes que presentaban puntuaciones en el cuartil o tercil superior tenían un riesgo aumentado de padecer ansiedad.

Aquellos que tuvieron las peores puntuaciones de bienestar emocional tuvieron el riesgo más elevado de padecer ansiedad cuando se los comparó con el resto de cuartiles (cuartil 4: OR: 65.00, p < 0.001). Las cuartiles superiores de las dimensiones de movilidad, actividades de la vida diaria, bienestar emocional y estado cognitivo aumentaron el riesgo de padecer ansiedad en 4.73 (OR: 4.73, p = 0.012); 5.20 (OR: 5.20, p = 0.039); 65 (OR: $65.00, \mathrm{p}<0.001)$ y 7.33 veces (OR: 7.33, $\mathrm{p}=0.034)$, respectivamente. Por el otro lado, los terciles superiores de las dimensiones de comunicación y estigmatización presentaban un riesgo aumentado de padecer ansiedad en $3.68(\mathrm{OR}: 3.68, \mathrm{p}=0.031)$ y 3.15 veces $(\mathrm{OR}$ : $3.15, \mathrm{p}=0.020$ ), respectivamente. No se encontró relación con las dimensiones de apoyo social ni con la de dolor (ver tabla 29). 
Tabla 29: Relación de la ansiedad y la calidad de vida según el PDQ-39.

\begin{tabular}{|c|c|c|c|c|}
\hline Calidad de Vida & Odds Ratio & $D S$ & $p$ valor & IC:95\% \\
\hline \multicolumn{5}{|c|}{ Movilidad (cuartiles) } \\
\hline 1 & 1.00 & & & \\
\hline 2 & 1.77 & 1.09 & 0.351 & $0.53-5.90$ \\
\hline 3 & 4.09 & 2.87 & 0.044 & $1.04-16.15$ \\
\hline 4 & 4.73 & 2.91 & 0.012 & $1.41-15.81$ \\
\hline \multicolumn{5}{|c|}{ Actividades de la vida diaria } \\
\hline 1 & 1.00 & & & \\
\hline 2 & 0.56 & 0.36 & 0.364 & $0.16-1.97$ \\
\hline 3 & 1.37 & 0.94 & 0.646 & $0.36-5.27$ \\
\hline 4 & 5.20 & 4.15 & 0.039 & $1.09-24.90$ \\
\hline \multicolumn{5}{|c|}{ Bienestar emocional } \\
\hline 1 & 1.00 & & & \\
\hline 2 & 3.25 & 2.12 & 0.071 & $0.90-11.70$ \\
\hline 3 & 13.00 & 9.87 & 0.001 & $2.93-57.61$ \\
\hline 4 & 65.00 & 74.59 & $<0.001$ & $6.86-616.21$ \\
\hline \multicolumn{5}{|l|}{ Estigmatización } \\
\hline 1 & 1.00 & & & \\
\hline 2 & 1.48 & 1.36 & 0.667 & $0.25-8.90$ \\
\hline 3 & 3.15 & 1.55 & 0.020 & $1.20-8.25$ \\
\hline \multicolumn{5}{|l|}{ Apoyo social } \\
\hline 1 & 1.00 & & & \\
\hline 2 & 2.20 & 1.30 & 0.179 & $0.70-7.00$ \\
\hline 3 & 0.98 & 0.51 & 0.970 & $0.35-2.72$ \\
\hline \multicolumn{5}{|l|}{ Estado cognitivo } \\
\hline 1 & 1.00 & & & \\
\hline 2 & 1.25 & 0.98 & 0.776 & $0.27-5.80$ \\
\hline 3 & 2.71 & 2.26 & 0.231 & $0.53-13.92$ \\
\hline 4 & 7.33 & 6.87 & 0.034 & $1.17-46.05$ \\
\hline \multicolumn{5}{|l|}{ Comunicación } \\
\hline 1 & 1.00 & & & \\
\hline 2 & 1.41 & 0.72 & 0.505 & $0.52-3.84$ \\
\hline 3 & 3.68 & 2.22 & 0.031 & $1.13-12.02$ \\
\hline \multicolumn{5}{|l|}{ Dolor } \\
\hline 1 & 1.00 & & & \\
\hline 2 & 0.48 & 0.32 & 0.275 & $0.13-1.80$ \\
\hline 3 & 3.69 & 2.76 & 0.080 & $0.86-15.94$ \\
\hline 4 & 3.76 & 2.54 & 0.050 & $1.00-14.10$ \\
\hline
\end{tabular}


Se calculó también la relación del PDSI con la ansiedad. Los resultados obtenidos se dividieron en cuartiles, siendo el cuartil 1 el que agrupaba a aquellos que tenían menor puntuación o mayor calidad de vida; mientras que, el cuartil 4 agrupaba a aquellos pacientes que obtuvieron puntuaciones más altas o que tenían peor calidad de vida. Se observó que aquellos pacientes pertenecientes al último cuartil presentaban 43.13 veces más riesgo de presentar niveles elevados de ansiedad que aquellos que estaban en el primer cuartil (OR: 43.13, $\mathrm{p}=0.001)$ (ver tabla 30).

Tabla 30: Relación del PDSI y ansiedad.

\begin{tabular}{ccccc}
\hline \hline $\begin{array}{c}\text { PDSI } \\
\text { (cuartiles) }\end{array}$ & Odds Ratio & DS & p valor & IC:95\% \\
\hline \hline 1 & 1.00 & & & \\
2 & 3.13 & 1.90 & 0.061 & $0.95-10.29$ \\
3 & 7.13 & 4.75 & 0.003 & $1.93-26.31$ \\
4 & 43.13 & 47.93 & 0.001 & $4.88-380.83$ \\
\hline \hline
\end{tabular}

Para ver la influencia de las variables más relevantes con la ansiedad se realizó un análisis de regresión logística (ver tabla 31).

Se encontró un OR inferior a la unidad en aquellos pacientes que no estaban casados, sin embargo esta relación no fue estadísticamente significativa.

Al analizar estado estado cognitivo se puede observar un aumento del riesgo de presentar niveles elevados de ansiedad a mayor deterioro cognitivo llegando a la significancia estadística en aquellos que presentan sospecha de deterioro cognitivo (OR: 82.86, $\mathrm{p}=0.030)$.

Por otro lado, se observa un aumento progresivo del riesgo de ansiedad a peor calidad de vida medida con el PDSI. El riesgo de presentar niveles de ansiedad elevados es 469.46 veces mayor en aquellos ubicados en el último cuartil del PDSI (OR: 469.46, p = 0.001). 
Tabla 31: Análisis de regresión logística múltiple de la ansiedad y otras variables.

\begin{tabular}{|c|c|c|c|c|}
\hline Variables & Odds Ratio & $D S$ & $p$ valor & $I C: 95 \%$ \\
\hline \multicolumn{5}{|l|}{ Sexo } \\
\hline Masculino & 1.00 & & & \\
\hline Femenino & 0.55 & 0.50 & 0.506 & $0.09-3.22$ \\
\hline \multicolumn{5}{|l|}{ Nivel Educativo } \\
\hline Superior & 1.00 & & & \\
\hline Ninguna & 8.52 & 15.04 & 0.225 & $0.27-270.97$ \\
\hline Primaria & 1.50 & 1.32 & 0.646 & $0.27-8.40$ \\
\hline Secundaria & 2.70 & 2.65 & 0.310 & $0.40-18.44$ \\
\hline \multicolumn{5}{|l|}{ Estado Civil } \\
\hline Casados & 1.00 & & & \\
\hline No casados & 0.53 & 0.50 & 0.501 & $0.08-3.38$ \\
\hline \multicolumn{5}{|l|}{ Tiempo de evolución (años) } \\
\hline$<3$ & 1.00 & & & \\
\hline$\geq 3 \mathrm{y}<6$ & 0.96 & 1.08 & 0.973 & $0.11-8.66$ \\
\hline$\geq 6 y \leq 10$ & 0.09 & 0.11 & 0.052 & $0.01-1.02$ \\
\hline$>10$ & 2.40 & 2.90 & 0.469 & $0.22-25.62$ \\
\hline \multicolumn{5}{|l|}{ Estadíos de Hoehn y Yahr } \\
\hline 0 & 1.00 & & & \\
\hline 1.0 & 2.54 & 4.85 & 0.627 & $0.06-107.82$ \\
\hline 1.5 & 0.38 & 0.85 & 0.665 & $0.01-29.30$ \\
\hline 2.0 & 17.86 & 40.66 & 0.205 & $0.21-1548.30$ \\
\hline 2.5 & 7.69 & 16.11 & 0.330 & $0.13-466.09$ \\
\hline 3.0 & 0.83 & 1.72 & 0.930 & $0.01-47.56$ \\
\hline 4.0 & 0.34 & 0.70 & 0.602 & $0.01-19.90$ \\
\hline \multicolumn{5}{|l|}{ Tratamiento oral + avanzado } \\
\hline 1 & 1.00 & & & \\
\hline 2 & 0.52 & 0.61 & 0.575 & $0.05-5.24$ \\
\hline 3 & 0.18 & 0.21 & 0.142 & $0.02-1.79$ \\
\hline$\geq 4$ & 0.18 & 0.25 & 0.212 & $0.01-2.66$ \\
\hline \multicolumn{5}{|l|}{ Estado cognitivo } \\
\hline Normal & 1.00 & & & \\
\hline Sospecha de patología & 82.86 & 168.50 & 0.030 & $1.54-4459.59$ \\
\hline Deterioro & 3.35 & 4.63 & 0.382 & $0.22-50.27$ \\
\hline \multicolumn{5}{|l|}{ PDSI } \\
\hline 1 & 1.00 & & & \\
\hline 2 & 24.64 & 26.28 & 0.003 & $3.05-199.32$ \\
\hline 3 & 27.04 & 30.44 & 0.003 & $2.98-245.66$ \\
\hline 4 & 469.46 & 862.11 & 0.001 & $12.84-17168.53$ \\
\hline
\end{tabular}




\subsection{Calidad de vida y su relación con las variables}

Las puntuaciones obtenidas en el PDQ-39 y el PDSI ya se describieron previamente y se pueden ver en la tabla 9. La figura 7 muestra las medias de las diversas dimensiones del PDQ-39 y el PDSI.

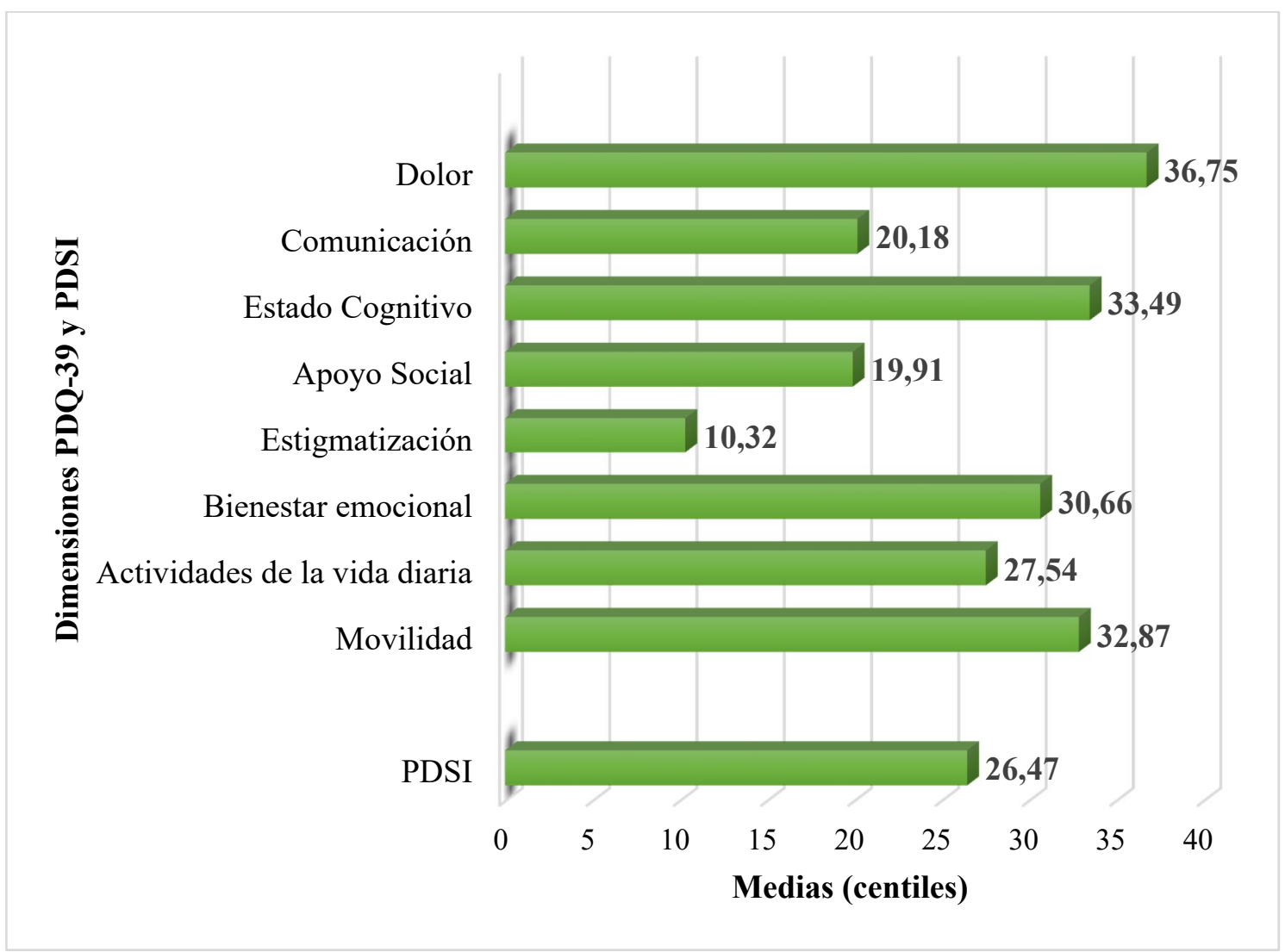

Figura 7: Puntuaciones medias de las dimensiones del PDQ-39 y el PDSI.

Diagrama de barras que muestra la distribución de las medias (en centiles) de las dimensiones del PDQ-39 y del PDSI observándose que la dimensión de dolor tiene la peor media y la estigmatización la menor. 
En esta parte del trabajo se busca encontrar como influyen las diversas variables, en especial la depresión y la ansiedad, en la calidad de vida por lo que se analiza cada una de las dimensiones del PDQ-39 y el PDSI. No se dividió las puntuaciones en cuartiles ni terciles.

Primero se muestran los datos de los análisis bivariados de cada dimensión y del PDSI y al final un resumen de análisis de regresión lineal múltiple.

\subsubsection{Calidad de Vida: Dimensión de Movilidad}

Respecto a la relación de la dimensión movilidad del PDQ-39 con las características generales de la población no se encontraron diferencias estadísticamente significativas con las variables sociodemográficas (sexo, nivel educativo, estado civil y sector de trabajo) ni con el estado cognitivo (ver tabla 32).

En la tabla 33 se puede ver la relación con diversas características de la enfermedad de Parkinson. Se encontró que un mayor estadío de la enfermedad impactaba negativamente en la dimensión de movilidad $(\mathrm{p}<0.001)$. No se encontró relación con el antecedente familiar de EP, la edad de inicio ni con el tiempo de evolución de la enfermedad.

No se encontró asociación de la movilidad y el número de tratamientos antiparkinsonianos recibidos (ver tabla 34). 
Tabla 32: Relación del PDQ-39: Movilidad y características generales.

\begin{tabular}{lcc}
\hline \hline Variables & $\begin{array}{c}\text { Media } \pm \text { DS/ Mediana } \\
(\boldsymbol{p} 25-\boldsymbol{p} 75)\end{array}$ & p valor \\
\hline \hline Sexo & $31.21 \pm 21.17$ & \\
Masculino & $35.26 \pm 26.80$ & 0.414 \\
$\quad$ Femenino & & \\
Nivel Educativo & $22.50(12.50-80.00)$ & \\
$\quad$ Ninguna & $38.75(16.88-50.00)$ & 0.563 \\
Primaria & $30.00(13.75-43.75)$ & \\
Secundaria & $15.00(6.25-47.50)$ & \\
$\quad$ Superior & & \\
Estado civil & $30.00(13.75-47.50)$ & \\
Casados & $36.25(6.88-53.12)$ & \\
$\quad$ No casados & & \\
Sector de trabajo & & \\
Primario & $30.00(18.75-36.25)$ & \\
Industrial & $36.25(21.88-48.12)$ & \\
Servicios & $23.75(10.00-47.50)$ & \\
Ama de casa & $47.50(13.12-61.25)$ & \\
Estado cognitivo & & \\
Normal & $30.88 \pm 22.74$ & \\
Sospecha de patología & $42.86 \pm 24.72$ & \\
Deterioro & $44.06 \pm 28.81$ & \\
\hline \hline
\end{tabular}


Tabla 33: Relación del PDQ-39: Movilidad y características de la Enfermedad de Parkinson.

\begin{tabular}{lcc}
\hline \hline Variables & $\begin{array}{c}\text { Media } \pm \text { DS } / \text { Mediana } \\
(\boldsymbol{p} 25-\boldsymbol{p} 75)\end{array}$ & p valor \\
\hline \hline Antecedente familiar & & \\
$\quad$ No & $35.00(15.00-48.75)$ & 0.182 \\
$\quad$ Sí & $15.00(10.00-45.62)$ & \\
Edad de inicio (años) & & \\
$\quad<50$ & $28.75 \pm 24.67$ & 0.562 \\
$\geq 50$ & $33.36 \pm 23.57$ & \\
Tiempo de evolución (años) & \\
$<3$ & $27.89 \pm 18.71$ & \\
$\geq 3$ y $<6$ & $30.82 \pm 24.65$ & \\
$\geq 6$ y $\leq 10$ & $29.17 \pm 21.84$ & \\
$>10$ & $43.33 \pm 26.24$ & \\
Estadíos de Hoehn $\boldsymbol{y}$ Yahr & \\
0 & $5.00(2.50-33.75)$ & \\
1.0 & $15.00(5.00-35.00)$ & \\
1.5 & $47.50(37.50-50.00)$ & \\
2.0 & $15.00(7.50-16.35)$ & \\
2.5 & $26.25(13.75-39.38)$ & \\
3.0 & $37.50(20.00-48.12)$ & \\
4.0 & $60.00(47.50-67.50)$ & \\
&
\end{tabular}


Tabla 34: Relación del PDQ-39: Movilidad y tratamientos antiparkinsonianos.

\begin{tabular}{lcc}
\hline \hline Variables & $\begin{array}{c}\text { Media } \pm \text { DS/Mediana } \\
(\boldsymbol{p} 25-\boldsymbol{p} 75)\end{array}$ & p valor \\
\hline \hline Tratamiento total & & \\
0 & $46.25(34.38-51.25)$ & \\
1 & $37.50(13.75-62.50)$ & 0.415 \\
2 & $25.00(6.25-46.25)$ & \\
3 & $30.00(13.12-47.50)$ & \\
$\geq 4$ & $30.00(18.75-60.00)$ & \\
Tratamiento oral & & \\
0 & $39.38 \pm 25.77$ & \\
1 & $41.79 \pm 32.04$ & \\
2 & $28.98 \pm 19.80$ & \\
3 & $29.91 \pm 20.87$ & \\
$\geq 4$ & $35.38 \pm 24.56$ & \\
Terapia avanzada & & \\
Ausente & $31.52 \pm 23.74$ & \\
Presente & $43.18 \pm 20.56$ & \\
\hline \hline
\end{tabular}

Como se muestra en la tabla 35, cuando se analizó la relación con otras patologías médicas se encontró que la presencia de patología cardiovascular influía negativamente en esta dimensión $(\mathrm{p}=0.023)$. Por otro lado, no se encontró asociación con pacientes que tenían el diagnóstico de alguna enfermedad psiquiátrica (ver tabla 36) ni con aquellos que se encontraban en tratamiento ansiolítico o antidepresivo (ver tabla 37). 
Tabla 35: Relación del PDQ-39: Movilidad y otras enfermedades médicas.

\begin{tabular}{ccc}
\hline \hline Enfermedades médicas & $\begin{array}{c}\text { Media } \pm \text { DS/ Mediana } \\
(\boldsymbol{p} 25-\boldsymbol{p} 75)\end{array}$ & p valor \\
\hline \hline Cardiovascular & & \\
Ausente & $28.31 \pm 23.44$ & 0.023 \\
Presente & $39.42 \pm 22.52$ & \\
Pulmonar & $32.83 \pm 24.30$ & 0.669 \\
Ausente & $36.39 \pm 15.11$ & \\
Presente & $33.22 \pm 23.84$ & 0.498 \\
Digestivo & $25.00 \pm 17.68$ & \\
Ausente & & \\
Presente & $33.14 \pm 23.63$ & \\
Oncológica & Ausente & \\
Presente & $26.88 \pm 25.44$ & 0.606 \\
Ausente & & \\
Presente & $28.80(7.50-46.25)$ & \\
\hline \hline
\end{tabular}

Tabla 36: Relación del PDQ-39: Movilidad y antecedente de patología de Salud Mental.

\begin{tabular}{lcc}
\hline \hline Enfermedades psiquiátricas & Media $\pm \boldsymbol{D S}$ & p valor \\
\hline \hline Ansiedad & & \\
$\quad$ Ausente & & \\
$\quad$ Presente & $32.47 \pm 23.88$ & 0.610 \\
Depresión & $36.72 \pm 21.55$ & \\
$\quad$ Ausente & & \\
$\quad$ Presente & $31.68 \pm 24.28$ & 0.328 \\
Otra & $37.63 \pm 20.52$ & \\
$\quad$ Ausente & & \\
Presente & $31.41 \pm 23.37$ & 0.243 \\
\hline \hline
\end{tabular}


Tabla 37: Relación del PDQ-39: Movilidad y tratamiento antidepresivo y ansiolítico.

\begin{tabular}{lll}
\hline \hline Tipo de tratamiento & Media $\pm \boldsymbol{D S}$ & $\boldsymbol{p}$ valor \\
\hline \hline & & \\
Tratamiento ansiolítico & & \\
No & $30.47 \pm 23.85$ & 0.216 \\
Sí & $36.64 \pm 23.01$ & \\
& & \\
Tratamiento antidepresivo & & \\
No & $30.29 \pm 23.48$ & 0.066 \\
Sí & $40.52 \pm 22.71$ & \\
\hline \hline
\end{tabular}

Se encontró una relación estadísticamente significativa en los pacientes que tenían sintomatología depresiva según el Beck-II o ansiedad según el STAI-R (ver tabla 38).

Tabla 38: Relación del PDQ-39: Movilidad y presencia de depresión o ansiedad.

\begin{tabular}{lll}
\hline \hline Variables & Media $\pm \boldsymbol{D S}$ & $\boldsymbol{p}$ valor \\
\hline \hline BDI-II & & \\
$\quad$ No depresión & & \\
Depresión & $26.45 \pm 20.56$ & $<0.001$ \\
& $46.15 \pm 24.22$ & \\
$\boldsymbol{S T \boldsymbol { A I } - \boldsymbol { R }}$ & & \\
$\quad$ No ansiedad & & \\
Ansiedad & $21.42 \pm 20.29$ & \\
& $38.16 \pm 23.27$ & \\
\hline \hline
\end{tabular}




\subsubsection{Calidad de Vida: Actividades de la vida diaria}

Respecto a la relación de la dimensión actividades de la vida diaria del PDQ-39 con las características generales, no se encontró relación con ninguna variable sociodemográfica estudiada ni el estado cognitivo (ver tabla 39). Sí se encontró relación $(\mathrm{p}<0.05)$ con aquellos que tenían mayor tiempo de evolución de la enfermedad y mayor estadío (ver tabla 40).

Tabla 39: Relación del PDQ-39: Actividades de la vida diaria y características generales.

\begin{tabular}{lcc}
\hline \hline Variables & $\begin{array}{c}\text { Media } \pm \text { DS } / \\
\text { Mediana }(\boldsymbol{p} 25-p 75)\end{array}$ & p valor \\
\hline \hline Sexo & $29.61 \pm 23.91$ & \\
$\quad$ Masculino & $24.57 \pm 27.98$ & 0.349 \\
$\quad$ Femenino & & \\
Nivel Educativo & $41.67(25.00-87.50)$ & \\
$\quad$ Ninguna & $20.83(4.17-42.71)$ & 0.421 \\
$\quad$ Primaria & $25.00(8.34-45.84)$ & \\
Secundaria & $12.50(4.17-35.42)$ & \\
$\quad$ Superior & & \\
Estado civil & $25.00(8.33-47.91)$ & 0.062 \\
$\quad$ Casados & $8.33(4.17-33.33)$ & \\
$\quad$ No casados & & \\
Sector de trabajo & & \\
Primario & & \\
Industrial & $25.00(14.59-33.34)$ & \\
Servicios & $35.41(11.46-63.54)$ & \\
Ama de casa & $14.59(4.17-41.67)$ & \\
Estado cognitivo & $18.75(5.21-33.33)$ & \\
$\quad$ Normal & & \\
Sospecha de patología & $38.69 \pm 32.16$ & \\
Deterioro & $34.90 \pm 23.56$ & \\
& & \\
\hline \hline
\end{tabular}


Tabla 40: Relación del PDQ-39: Actividades de la vida diaria y características de la Enfermedad de Parkinson.

\begin{tabular}{lcc}
\hline \hline Variables & Mediana (p25-p75) & p valor \\
\hline \hline Antecedente familiar & & \\
$\quad$ No & $25.00(8.33-47.91)$ & 0.054 \\
$\quad$ Sí & $12.50(3.13-29.17)$ & \\
Edad de inicio (años) & & \\
$\quad<50$ & $12.50(8.33-39.59)$ & 0.995 \\
$\geq 50$ & $20.83(4.17-41.67)$ & \\
Tiempo de evolución (años) & & \\
$<3$ & $20.83(4.17-45.84)$ & \\
$\geq 3$ y $<6$ & $22.91(5.21-33.33)$ & 0.039 \\
$\geq 6$ y $\leq 10$ & $16.67(0.00-29.17)$ & \\
$>10$ & $41.67(8.33-63.54)$ & \\
Estadíos de Hoehn y Yahr & & \\
0 & $0.00(0.00-12.50)$ & \\
1.0 & $4.17(4.17-25.00)$ & \\
1.5 & $25.00(20.83-41.67)$ & \\
2.0 & $8.33(0.00-18.75)$ & $<0.001$ \\
2.5 & $20.83(5.21-29.17)$ & \\
3.0 & $37.50(16.67-56.25)$ & \\
4.0 & $45.83(29.17-66.67)$ & \\
& & \\
\hline \hline
\end{tabular}

No se encontró relación entre la dimensión de actividades de la vida diaria y el número de tratamientos antiparkinsonianos recibidos (ver tabla 41) 
Tabla 41: Relación del PDQ-39: Actividades de la vida diaria y tratamientos antiparkinsonianos.

\begin{tabular}{lcc}
\hline \hline Variables & $\begin{array}{c}\text { Media } \pm \text { DS } / \\
\text { Mediana }(\boldsymbol{p} 25-\boldsymbol{p} 75)\end{array}$ & p valor \\
\hline \hline Tratamiento total & $14.59(3.13-34.38)$ & \\
0 & $45.83(18.75-60.41)$ & \\
1 & $20.83(2.08-39.59)$ & 0.238 \\
2 & $18.75(8.33-36.46)$ & \\
3 & $16.67(4.17-39.59)$ & \\
$\geq 4$ & & \\
Tratamiento oral & $14.59(3.13-34.38)$ & \\
0 & $45.83(25.00-58.33)$ & \\
1 & $20.83(4.17-44.80)$ & 0.093 \\
2 & $12.50(4.17-29.17)$ & \\
3 & $25.00(0.00-41.67)$ & \\
$\geq 4$ & & \\
Terapia avanzada & $26.14 \pm 25.84$ & \\
Ausente & $38.26 \pm 22.19$ & \\
Presente & \\
\hline \hline
\end{tabular}

Al analizar la relación con otras enfermedades médicas, se encontró asociación entre la presencia de patología pulmonar y la dimensión de actividades de la vida diaria del PDQ39 (ver tabla 42).

No se encontró relación con antecedente de patología de salud mental ni con la toma de ansiolíticos y antidepresivos (ver tablas 43 y 44 ). 
Tabla 42: Relación del PDQ-39: Actividades de la vida diaria y otras enfermedades médicas.

\begin{tabular}{lcc}
\hline \hline Enfermedades médicas & $\begin{array}{c}\text { Media } \pm \text { DS/Mediana } \\
(\boldsymbol{p} 25-\boldsymbol{p} 75)\end{array}$ & p valor \\
\hline \hline Cardiovascular & & \\
Ausente & $25.15 \pm 26.40$ & 0.278 \\
Presente & $30.98 \pm 24.42$ & \\
Pulmonar & & \\
Ausente & $25.88 \pm 25.23$ & 0.026 \\
Presente & $45.83 \pm 23.75$ & \\
Digestivo & & \\
Ausente & $28.07 \pm 25.85$ & \\
Presente & $15.63 \pm 18.75$ & \\
Oncológica & & \\
Ausente & $28.39 \pm 25.79$ & \\
Presente & $8.34 \pm 11.28$ & \\
Otras & & \\
Ausente & & \\
Presente & $24.07(4.17-37.50)$ & \\
\hline \hline
\end{tabular}

Tabla 43: Relación del PDQ-39: Actividades de la vida diaria y antecedente de patología de Salud Mental.

\begin{tabular}{lcc}
\hline \hline Enfermedades psiquiátricas & Media $\pm \boldsymbol{D S}$ & p valor \\
\hline \hline Ansiedad & & \\
$\quad$ Ausente & $27.18 \pm 25.76$ & 0.671 \\
Presente & $31.02 \pm 25.61$ & \\
Depresión & & \\
Ausente & $27.03 \pm 25.97$ & 0.697 \\
Presente & $29.61 \pm 24.84$ & \\
Otra & & \\
Ausente & $27.17 \pm 26.81$ & \\
Presente & $28.96 \pm 21.22$ & \\
\hline \hline
\end{tabular}


Tabla 44: Relación del PDQ-39: Actividades de la vida diaria y tratamiento antidepresivo y ansiolítico.

\begin{tabular}{lcc}
\hline \hline Tipo de tratamiento & $\begin{array}{c}\text { Media } \pm \text { DS/Mediana } \\
(\boldsymbol{p} 25-p 75)\end{array}$ & p valor \\
\hline \hline & & \\
Tratamiento ansiolítico & $25.93 \pm 26.62$ & 0.446 \\
No & $30.07 \pm 24.16$ & \\
Sí & & \\
& & 0.357 \\
Tratamiento antidepresivo & $20.83(4.17-41.67)$ & \\
No & $25.00(10.42-56.25)$ & \\
Sí & & \\
\hline \hline
\end{tabular}

Se encontró una relación estadísticamente significativa en los pacientes que tenían sintomatología depresiva según el Beck-II o ansiedad según el STAI-R (ver tabla 45).

Tabla 45: Relación del PDQ-39: Actividades de la vida diaria y presencia de depresión o ansiedad.

\begin{tabular}{lcc}
\hline \hline Variables & Media $\pm \boldsymbol{D S}$ & $\boldsymbol{p}$ valor \\
\hline \hline & & \\
$\boldsymbol{B D I}-\boldsymbol{I I}$ & $19.92 \pm 19.72$ & \\
$\quad$ No depresión & $43.28 \pm 29.38$ & $<0.001$ \\
Depresión & & \\
$\boldsymbol{S T A I - R}$ & $15.42 \pm 16.64$ & $<0.001$ \\
No ansiedad & $33.14 \pm 27.19$ & \\
Ansiedad & & \\
\hline \hline
\end{tabular}




\subsubsection{Calidad de Vida: Bienestar emocional}

Se analizaron diversas variables sociodemográficas, encontrándose que el sexo femenino tenía un impacto negativo con la dimensión de bienestar emocional $(\mathrm{p}=0.003)$. No se encontró relación con el nivel educativo, el estado ni el sector de trabajo. Tampoco se encontró relación con el estado cognitivo medido por el MEC-30 (ver tabla 46).

Tabla 46: Relación del PDQ-39: Bienestar emocional y las diversas variables.

\begin{tabular}{lcc}
\hline \hline Variables & $\begin{array}{c}\text { Media } \pm \text { DS/Mediana } \\
(\boldsymbol{p} 25-\boldsymbol{p} 75)\end{array}$ & p valor \\
\hline \hline Sexo & & \\
Masculino & $25.52 \pm 17.71$ & 0.003 \\
Femenino & $38.03 \pm 22.90$ & \\
Nivel Educativo & & \\
Ninguna & $20.83(12.50-58.33)$ & \\
Primaria & $29.17(12.50-45.83)$ & 0.919 \\
Secundaria & $29.17(16.67-43.75)$ & \\
Superior & $33.33(14.59-41.67)$ & \\
Estado civil & & \\
Casados & $29.10 \pm 20.02$ & \\
No casados & $34.37 \pm 22.61$ & \\
Sector de trabajo & & \\
Primario & $29.17(10.41-39.59)$ & \\
Industrial & $18.75(8.33-42.71)$ & \\
Servicios & $25.00(13.54-44.79)$ & \\
Ama de casa & $39.59(29.17-53.13)$ & \\
Estado cognitivo & & \\
Normal & $29.37 \pm 19.38$ & \\
Sospecha de patología & $34.53 \pm 23.78$ & \\
Deterioro & $40.10 \pm 31.09$ & \\
\hline \hline
\end{tabular}


Como se observa en la tabla 47, se estudió diversas variables de la EP como la presencia de antecedentes familiares de esta patología, la edad de inicio, el tiempo de evolución y el estadío clínico de la enfermedad, encontrándose asociación solamente con el estadío de Hoehn y Yahr $(\mathrm{p}=0.039)$.

Tabla 47: Relación del PDQ-39: Bienestar emocional y características de la Enfermedad de Parkinson.

\begin{tabular}{lcc}
\hline \hline Variables & $\begin{array}{c}\text { Media } \pm \text { DS/Mediana } \\
(\boldsymbol{p} 25-\boldsymbol{p} 75)\end{array}$ & p valor \\
\hline \hline Antecedente familiar & & \\
$\quad$ No & $32.47 \pm 22.36$ & 0.233 \\
$\quad$ Sí & $26.79 \pm 16.84$ & \\
Edad de inicio (años) & & \\
$\quad<50$ & $27.50 \pm 19.26$ & 0.615 \\
$\geq 50$ & $31.03 \pm 21.08$ & \\
Tiempo de evolución (años) & $27.85 \pm 22.95$ & \\
$<3$ & $33.14 \pm 19.90$ & \\
$\geq 3$ y $<6$ & $26.67 \pm 17.70$ & \\
$\geq 6$ y $\leq 10$ & $35.59 \pm 23.38$ & \\
$>10$ & & \\
Estadíos de Hoehn $\boldsymbol{y}$ Yahr & $0.00(0.00-6.25)$ & \\
0 & $25.00(8.33-45.83)$ & \\
1.0 & $41.67(12.50-58.33)$ & \\
1.5 & $20.83(6.25-29.16)$ & \\
2.0 & $37.50(34.37-44.79)$ & \\
2.5 & $22.91(16.67-38.54)$ & \\
3.0 & $33.33(16.67-54.17)$ & \\
4.0 & & \\
\hline \hline
\end{tabular}


$\mathrm{Al}$ analizar la dimensión bienestar emocional con la cantidad de tratamientos recibidos o con la presencia de terapia avanzada, no se encontró asociación entre ellas (ver tablas 48).

Tabla 48: Relación del PDQ-39: Bienestar emocional y tratamientos antiparkinsonianos.

\begin{tabular}{lcc}
\hline \hline Variables & $\begin{array}{c}\text { Media } \pm \text { DS } / \text { Mediana } \\
(\boldsymbol{p} 25-\boldsymbol{p} 75)\end{array}$ & p valor \\
\hline \hline Tratamiento total & & \\
0 & $39.59(28.12-42.71)$ & \\
1 & $25.00(14.59-56.25)$ & \\
2 & $20.83(14.59-47.91)$ & 0.985 \\
3 & $31.25(16.67-41.67)$ & \\
$\geq 4$ & $33.33(10.41-37.50)$ & \\
Tratamiento oral & & \\
0 & $39.59(28.12-42.71)$ & \\
1 & $25.00(16.67-58.33)$ & \\
2 & $25.00(12.50-45.83)$ & \\
3 & $33.33(16.67-41.67)$ & \\
$\geq 4$ & $29.17(8.33-37.50)$ & \\
Terapia avanzada & & \\
Ausente & $30.21 \pm 21.28$ & \\
Presente & $34.09 \pm 17.46$ & \\
\end{tabular}

Se buscó la influencia de otras enfermedades médicas en el bienestar emocional así como de historia de enfermedades de Salud Mental ya diagnosticadas, encontrándose un impacto negativo de la historia de depresión sobre esta dimensión. No se encontró asociación con el resto de patologías (ver tablas 49 y 50). 
Tabla 49: Relación del PDQ-39: Bienestar emocional diaria y otras enfermedades médicas.

\begin{tabular}{lcc}
\hline \hline Enfermedades médicas & Media \pm DS & p valor \\
\hline \hline $\begin{array}{l}\text { Cardiovascular } \\
\text { Ausente } \\
\quad \text { Presente }\end{array}$ & $30.21 \pm 19.71$ & \\
$\begin{array}{l}\text { Pulmonar } \\
\text { Ausente } \\
\quad \text { Presente }\end{array}$ & $31.30 \pm 22.60$ & 0.803 \\
$\begin{array}{l}\text { Digestivo } \\
\quad \text { Ausente } \\
\quad \begin{array}{l}\text { Presente } \\
\text { Oncológica }\end{array}\end{array}$ & $30.88 \pm 21.40$ & 0.985 \\
$\quad \begin{array}{l}\text { Ausente } \\
\text { Presente }\end{array}$ & $31.02 \pm 15.04$ & \\
Otras & $30.49 \pm 20.95$ & 0.717 \\
$\quad \begin{array}{l}\text { Ausente } \\
\text { Presente }\end{array}$ & $34.38 \pm 20.24$ & \\
\hline \hline
\end{tabular}

Tabla 50: Relación del PDQ-39: Bienestar emocional y antecedente de patología de Salud Mental.

\begin{tabular}{lcc}
\hline \hline Enfermedades psiquiátricas & Media $\pm \boldsymbol{D S}$ & p valor \\
\hline \hline Ansiedad & & \\
$\quad \begin{array}{l}\text { Ausente } \\
\text { Presente }\end{array}$ & $29.55 \pm 20.98$ & 0.111 \\
Depresión & $41.20 \pm 16.85$ & \\
$\quad \begin{array}{l}\text { Ausente } \\
\text { Presente }\end{array}$ & $27.58 \pm 20.13$ & 0.003 \\
Otros & $42.98 \pm 19.45$ & \\
$\quad$ Ausente & & \\
Presente & $31.11 \pm 20.37$ & 0.684 \\
\hline \hline
\end{tabular}


Los pacientes que estaban en tratamiento antidepresivo presentaban peor bienestar emocional, no encontrándose relación con aquellas que estaban en tratamiento con benzodiacepinas o análogos (ver tabla 51).

Tabla 51: Relación del PDQ-39: Bienestar emocional y tratamiento antidepresivo y ansiolítico.

\begin{tabular}{ccc}
\hline \hline Tipo de tratamiento & Media \pm DS & p valor \\
\hline \hline & & \\
\multicolumn{2}{c}{ Tratamiento ansiolítico } & \\
No & $28.09 \pm 19.95$ & 0.133 \\
Sí & $34.68 \pm 21.81$ & \\
Tratamiento antidepresivo & & \\
No & $26.12 \pm 19.82$ & $<0.001$ \\
Sí & $44.10 \pm 18.05$ & \\
\hline \hline
\end{tabular}

Se encontró una asociación fuerte entre la presencia de depresión y ansiedad con un deterioro del bienestar emocional $(p<0.001)$ (ver tabla 52).

Tabla 52: Relación del PDQ-39: Bienestar emocional y presencia de depresión o ansiedad.

\begin{tabular}{lcc}
\hline \hline Variables & Media $\pm \boldsymbol{D S}$ & p valor \\
\hline \hline & & \\
$\boldsymbol{B D I}$-II & & \\
$\quad$ No depresión & $22.40 \pm 15.30$ & $<0.001$ \\
$\quad$ Depresión & $47.71 \pm 20.55$ & \\
$\boldsymbol{S T \boldsymbol { A I I } - \boldsymbol { R }}$ & & $<0.001$ \\
$\quad$ No ansiedad & $15.42 \pm 13.36$ & \\
$\quad$ Ansiedad & $37.69 \pm 19.93$ & \\
\hline \hline
\end{tabular}




\subsubsection{Calidad de Vida: Estigmatización}

Respecto a la relación de la dimensión estigmatización del PDQ-39 con las características generales de la población, no se encontró asociación estadísticamente significativa con ninguna de ellas (ver tabla 53). Tampoco se encontró asociación con el antecedente familiar de EP, la edad de inicio de la enfermedad, el tiempo de evolución, el estadío de la enfermedad ni la cantidad de tratamiento específico recibido (ver tablas 54 y 55).

Tabla 53: Relación del PDQ-39: Estigmatización y características generales.

\begin{tabular}{lcc}
\hline \hline Variables & $\begin{array}{c}\text { Media } \pm \text { DS/ Mediana } \\
(\boldsymbol{p} 25-\boldsymbol{p} 75)\end{array}$ & $\boldsymbol{p}$ valor \\
\hline \hline Sexo & & \\
Masculino & $8.37 \pm 10.27$ & 0.125 \\
$\quad$ Femenino & $13.14 \pm 17.13$ & \\
Nivel Educativo & & \\
$\quad$ Ninguna & $12.50(0.00-12.50)$ & \\
Primaria & $0.00(0.00-14.06)$ & \\
Secundaria & $6.25(0.00-25.00)$ & \\
Superior & $12.50(0.00-12.50)$ & \\
Estado civil & & \\
Casados & $0.00(0.00-12.50)$ & \\
$\quad$ No casados & $9.38(0.00-25.00)$ & \\
Sector de trabajo & & \\
Primario & $0.00(0.00-9.38)$ & \\
Industrial & $6.25(0.00-12.50)$ & \\
Servicios & $6.25(0.00-12.50)$ & \\
Ama de casa & $3.12(0.00-23.44)$ & \\
Estado cognitivo & & \\
Normal & $0.00(0.00-12.50)$ & \\
Sospecha de patología & $0.00(0.00-9.38)$ & \\
Deterioro & $0.00(0.00-12.50)$ & \\
\hline \hline
\end{tabular}


Tabla 54: Relación del PDQ-39: Estigmatización y características de la Enfermedad de Parkinson.

\begin{tabular}{lcc}
\hline \hline Variables & Mediana (p25-p75) & p valor \\
\hline \hline Antecedente familiar & & \\
$\quad$ No & $0.00(0.00-12.50)$ & 0.691 \\
$\quad$ Sí & $6.25(0.00-14.06)$ & \\
Edad de inicio (años) & & \\
$\quad<50$ & $12.50(0.00-12.50)$ & 0.790 \\
$\geq 50$ & $0.00(0.00-12.50)$ & \\
Tiempo de evolución (años) & $0.00(0.00-15.62)$ & \\
$<3$ & $6.25(0.00-17.19)$ & \\
$\geq 3$ y $<6$ & $0.00(0.00-12.50)$ & \\
$\geq 6$ y $\leq 10$ & $12.50(0.00-14.06)$ & \\
$>10$ & & \\
Estadíos de Hoehn $\boldsymbol{y}$ Yahr & $0.00(0.00-21.88)$ & \\
0 & $6.25(0.00-18.75)$ & \\
1.0 & $12.50(0.00-25.00)$ & \\
1.5 & $0.00(0.00-18.75)$ & \\
2.0 & $6.25(0.00-12.50)$ & \\
2.5 & $0.00(0.00-12.50)$ & \\
3.0 & $12.50(0.00-18.75)$ & \\
4.0 & & \\
& & \\
\hline \hline
\end{tabular}


Tabla 55: Relación del PDQ-39: Estigmatización y tratamientos antiparkinsonianos.

\begin{tabular}{lcc}
\hline \hline Variables & Mediana $(\boldsymbol{p 2 5}-\boldsymbol{p} 75)$ & $\boldsymbol{p}$ valor \\
\hline \hline Tratamiento total & & \\
0 & $0.00(0.00-3.12)$ & \\
1 & $6.25(0.00-12.50)$ & \\
2 & $12.50(0.00-28.12)$ & 0.487 \\
3 & $9.38(0.00-18.75)$ & \\
$\geq 4$ & $0.00(0.00-12.50)$ & \\
Tratamiento oral & & \\
0 & $0.00(0.00-3.12)$ & \\
1 & $6.25(0.00-12.50)$ & \\
2 & $12.50(0.00-20.31)$ & \\
3 & $0.00(0.00-18.75)$ & \\
$\geq 4$ & $0.00(0.00-12.50)$ & \\
Terapia avanzada & & \\
Ausente & $0.00(0.00-14.06)$ & \\
Presente & $12.50(0.00-12.50)$ & \\
\hline \hline
\end{tabular}

Se buscó la influencia de la otras patologías médicas en la estigmatización que puede sentir el paciente, no encontrándose ninguna asociación (ver tabla 56).

Por otro lado, tampoco se encontró asociación entre los pacientes que ya tenían algún diagnóstico de patología psiquiátrica ni con la toma de tratamiento antidepresivo o ansiolítico (ver tablas 57 y 58). 
Tabla 56: Relación del PDQ-39: Estigmatización y otras enfermedades médicas.

\begin{tabular}{lcc}
\hline \hline \multicolumn{1}{c}{ Enfermedades médicas } & $\begin{array}{c}\text { Media } \pm \text { DS/Mediana } \\
(\boldsymbol{p} 25-\boldsymbol{p} 75)\end{array}$ & p valor \\
\hline \hline Cardiovascular & & \\
Ausente & $12.17 \pm 14.22$ & 0.116 \\
Presente & $7.69 \pm 12.46$ & \\
Pulmonar & & \\
Ausente & $10.88 \pm 14.15$ & 0.116 \\
Presente & $6.25 \pm 6.99$ & \\
Digestivo & & \\
Ausente & $6.25(0.00-12.50)$ & \\
Presente & $0.00(0.00-9.38)$ & \\
Oncológica & & \\
Ausente & $6.25(0.00-15.62)$ & \\
Presente & $0.00(0.00-3.12)$ & \\
Otras & & \\
Ausente & $12.50(0.00-18.75)$ & \\
Presente & $0.00(0.00-12.50)$ & \\
\hline \hline
\end{tabular}

Tabla 57: Relación del PDQ-39: Estigmatización y antecedente de patología de Salud Mental.

\begin{tabular}{lcc}
\hline \hline Enfermedades psiquiátricas & Mediana $(\mathbf{p} 25-\boldsymbol{p} 75)$ & p valor \\
\hline \hline Ansiedad & & \\
$\quad$ Ausente & $0.00(0.00-12.50)$ & 0.317 \\
$\quad \begin{array}{l}\text { Presente } \\
\text { Depresión } \\
\text { Ausente } \\
\text { Presente }\end{array}$ & $12.50(0.00-18.75)$ & \\
$\begin{array}{l}\text { Otros } \\
\text { Ausente } \\
\text { Presente }\end{array}$ & $3.12(0.00-12.50)$ & 0.540 \\
\hline \hline
\end{tabular}


Tabla 58: Relación del PDQ-39: Estigmatización y tratamiento antidepresivo y ansiolítico.

\begin{tabular}{ccc}
\hline \hline Tipo de tratamiento & $\begin{array}{c}\text { Media } \pm \text { DS/Mediana } \\
(\mathbf{p 2 5}-\mathbf{p 7 5})\end{array}$ & p valor \\
\hline \hline & & \\
Tratamiento ansiolítico & $10.78 \pm 14.09$ & \\
No & $9.63 \pm 13.06$ & 0.691 \\
Sí & & \\
Tratamiento antidepresivo & $0.00(0.00-12.50)$ & 0.159 \\
No & $12.50(0.00-18.75)$ & \\
Sí & & \\
\hline \hline
\end{tabular}

Se encontró asociación estadísticamente significativa entre aquellos pacientes que presentaron peores puntuaciones en la dimensión de estigmatización y los que presentaban depresión o ansiedad según los cuestionarios de Beck-II y STAI-R (ver tabla 59).

Tabla 59: Relación del PDQ-39: Estigmatización y presencia de depresión o ansiedad.

\begin{tabular}{lcc}
\hline \hline Variables & Media $\pm \boldsymbol{D S}$ & p valor \\
\hline \hline & & \\
$\boldsymbol{B D I} \boldsymbol{I I}$ & & \\
$\quad$ No depresión & $7.23 \pm 10.46$ & 0.007 \\
$\quad$ Depresión & $16.73 \pm 17.03$ & \\
$\boldsymbol{S T} \boldsymbol{A} \boldsymbol{A I}-\boldsymbol{R}$ & & \\
$\quad$ No ansiedad & $5.21 \pm 9.15$ & 0.003 \\
$\quad$ Ansiedad & $12.69 \pm 14.74$ & \\
\hline \hline
\end{tabular}




\subsubsection{Calidad de Vida: Apoyo social.}

La tabla 60 muestra los resultados del análisis de la dimensión apoyo social y diversas variables sociodemográficas y el estado cognitivo, no encontrándose ninguna relación estadísticamente significativa.

Tabla 60: Relación del PDQ-39: Apoyo social y características generales.

\begin{tabular}{|c|c|c|}
\hline Variables & $\begin{array}{c}\text { Media } \pm \text { DS } / \text { Mediana } \\
(p 25-p 75)\end{array}$ & p valor \\
\hline \multicolumn{3}{|l|}{ Sexo } \\
\hline Masculino & $21.88 \pm 23.81$ & \multirow{2}{*}{0.320} \\
\hline Femenino & $17.09 \pm 21.54$ & \\
\hline \multicolumn{3}{|l|}{ Nivel Educativo } \\
\hline Ninguna & $16.67(0.00-25.00)$ & \multirow{4}{*}{0.494} \\
\hline Primaria & $25.00(0.00-33.33)$ & \\
\hline Secundaria & $8.33(0.00-25.00)$ & \\
\hline Superior & $16.67(0.00-25.00)$ & \\
\hline \multicolumn{3}{|l|}{ Estado civil } \\
\hline Casados & $16.67(0.00-25.00)$ & \multirow{2}{*}{0.910} \\
\hline No casados & $16.67(0.00-33.33)$ & \\
\hline \multicolumn{3}{|l|}{ Sector de trabajo } \\
\hline Primario & $16.67(4.17-33.34)$ & \multirow{4}{*}{0.365} \\
\hline Industrial & $25.00(0.00-50.00)$ & \\
\hline Servicios & $8.33(0.00-25.00)$ & \\
\hline Ama de casa & $16.66(0.00-33.33)$ & \\
\hline \multicolumn{3}{|l|}{ Estado cognitivo } \\
\hline Normal & $16.67(0.00-25.00)$ & \multirow{3}{*}{0.332} \\
\hline Sospecha de patología & $0.00(0.00-12.50)$ & \\
\hline Deterioro & $33.33(0.00-33.33)$ & \\
\hline
\end{tabular}


Se encontró que las personas cuyo inicio de la EP fue $\geq 50$ años de edad, presentaban una relación inversa con la sensación de apoyo social recibido $(\mathrm{p}=0.013)$. No se encontró relación con la historia familiar, el tiempo de evolución ni el estadío de la EP (ver tabla 61). Tampoco se encontró asociación con la cantidad de tratamientos antiparkinsonianos recibidos, ni con el haber recibido o no una terapia avanzada (ver tabla 62).

Tabla 61: Relación del PDQ-39: Apoyo social y características de la Enfermedad de Parkinson.

\begin{tabular}{lcc}
\hline \hline Variables & Mediana (p25-p75) & p valor \\
\hline \hline Antecedente familiar & & \\
$\quad$ No & $16.67(0.00-29.16)$ & 0.627 \\
$\quad$ Sí & $16.67(0.00-33.33)$ & \\
Edad de inicio (años) & $0.00(0.00-6.25)$ & 0.013 \\
$\quad<50$ & $16.67(0.00-33.33)$ & \\
$\geq 50$ & \\
Tiempo de evolución (años) & $8.33(0.00-16.67)$ & \\
$<3$ & $25.00(10.42-45.83)$ & 0.069 \\
$\geq 3$ y $<6$ & $16.67(0.00-33.33)$ & \\
$\geq 6$ y $\leq 10$ & $8.33(0.00-25.00)$ & \\
$>10$ & \\
Estadíos de Hoehn $\boldsymbol{y}$ Yahr & $8.33(4.17-50.00)$ & \\
0 & $8.33(0.00-25.00)$ & \\
1.0 & $16.67(0.00-50.00)$ & \\
1.5 & $0.00(0.00-16.66)$ & \\
2.0 & $29.16(16.67-39.59)$ & \\
2.5 & $0.00(0.00-25.00)$ & \\
3.0 & $25.00(8.33-33.33)$ & \\
4.0 & & \\
&
\end{tabular}


Tabla 62: Relación del PDQ-39: Apoyo social y tratamientos antiparkinsonianos.

\begin{tabular}{lcc}
\hline \hline Variables & Mediana $(\mathbf{p 2 5}-\mathbf{p} 75)$ & p valor \\
\hline \hline Tratamiento total & & \\
0 & $16.67(0.00-29.16)$ & \\
1 & $0.00(0.00-33.33)$ & 0.425 \\
2 & $16.67(0.00-25.00)$ & \\
3 & $16.67(0.00-29.16)$ & \\
$\geq 4$ & & \\
Tratamiento oral & $25.00(22.92-14.67)$ & \\
0 & $16.67(0.00-25.00)$ & \\
1 & $4.17(0.00-33.33)$ & 0.304 \\
2 & $16.67(8.33-25.00)$ & \\
3 & $16.67(0.00-25.00)$ & \\
$\geq 4$ & & \\
Terapia avanzada & $16.67(0.00-33.33)$ & \\
Ausente & $0.00(0.00-29.16)$ & \\
Presente & & \\
&
\end{tabular}

No se encontró influencia de otras enfermedades orgánicas con la dimensión de apoyo social (ver tabla 63). Al analizar la influencia de haber tenido el diagnóstico de alguna patología de salud mental, se encontró que aquellos que no habían sido diagnosticados previamente de ansiedad se relacionaban negativamente con la dimensión de apoyo social (ver tabla 64). 
Tabla 63: Relación del PDQ-39: Apoyo social y otras enfermedades médicas.

\begin{tabular}{|c|c|c|}
\hline Enfermedades médicas & $\begin{array}{c}\text { Media } \pm \text { DS/ Mediana } \\
(p 25-p 75)\end{array}$ & p valor \\
\hline \multicolumn{3}{|l|}{ Cardiovascular } \\
\hline Ausente & $18.30 \pm 21.87$ & \multirow{2}{*}{0.415} \\
\hline Presente & $22.22 \pm 24.43$ & \\
\hline \multicolumn{3}{|l|}{ Pulmonar } \\
\hline Ausente & $16.67(0.00-33.33)$ & \multirow{2}{*}{0.379} \\
\hline Presente & $8.33(0.00-16.67)$ & \\
\hline \multicolumn{3}{|l|}{ Digestivo } \\
\hline Ausente & $19.41 \pm 22.64$ & \multirow{2}{*}{0.314} \\
\hline Presente & $31.25 \pm 29.95$ & \\
\hline \multicolumn{3}{|l|}{ Oncológica } \\
\hline Ausente & $20.24 \pm 23.28$ & \multirow{2}{*}{0.511} \\
\hline Presente & $12.50 \pm 10.76$ & \\
\hline \multicolumn{3}{|l|}{ Otras } \\
\hline Ausente & $8.33(0.00-25.00)$ & \multirow{2}{*}{0.449} \\
\hline Presente & $16.67(0.00-33.33)$ & \\
\hline
\end{tabular}

Tabla 64: Relación del PDQ-39: Apoyo social y antecedente de patología de Salud Mental.

\begin{tabular}{lcc}
\hline \hline Enfermedades psiquiátricas & $\begin{array}{c}\text { Media } \pm \text { DS/Mediana } \\
(\boldsymbol{p} 25-\boldsymbol{p} 75)\end{array}$ & p valor \\
\hline \hline Ansiedad & & \\
$\quad$ Ausente & $21.12 \pm 23.59$ & 0.004 \\
$\quad$ Presente & $8.33 \pm 9.32$ & \\
Depresión & & \\
$\quad$ Ausente & $19.08 \pm 23.72$ & 0.481 \\
$\quad$ Presente & $23.25 \pm 19.56$ & \\
Otros & & \\
$\quad$ Ausente & $16.67(0.00-25.00)$ & 0.582 \\
Presente & $20.84(0.00-37.50)$ & \\
\hline \hline
\end{tabular}


No se encontró relación del apoyo social con la toma de tratamiento ansiolítico ni antidepresivo (ver tabla 65). Tampoco se encontró relación con las personas que tenían depresión y ansiedad según los cuestionarios de Beck-II y STAI-R (ver tabla 66).

Tabla 65: Relación del PDQ-39: Apoyo social y tratamiento antidepresivo y ansiolítico.

\begin{tabular}{ccc}
\hline \hline Tipo de tratamiento & $\begin{array}{c}\text { Media } \pm \text { DS/Mediana } \\
(\boldsymbol{p} 25-\boldsymbol{p} 75)\end{array}$ & $\boldsymbol{p}$ valor \\
\hline \hline & & \\
Tratamiento ansiolítico & & \\
No & $20.40 \pm 23.73$ & 0.796 \\
Sí & $19.14 \pm 21.86$ & \\
Tratamiento antidepresivo & & \\
No & $8.33(0.00-25.00)$ & 0.534 \\
Sí & $16.67(0.00-33.33)$ & \\
\hline \hline
\end{tabular}

Tabla 66: Relación del PDQ-39: Apoyo social y presencia de depresión o ansiedad.

\begin{tabular}{lcc}
\hline \hline Variables & Media $\pm \boldsymbol{D S}$ & $\boldsymbol{p}$ valor \\
\hline \hline & & \\
BDI-II & & \\
$\quad$ No Depresión & $17.58 \pm 20.69$ & 0.155 \\
$\quad$ Depresión & $24.73 \pm 26.66$ & \\
STAI-R & & \\
No ansiedad & $18.33 \pm 21.26$ & 0.651 \\
$\quad$ Ansiedad & $20.64 \pm 23.76$ & \\
\hline
\end{tabular}




\subsubsection{Calidad de Vida: Estado cognitivo}

En el análisis bivariado se encontró relación de una peor percepción del estado cognitivo (medido por la dimensión estado cognitivo del PDQ-39) con el sexo femenino $(p=0.003)$. No se encontró asociación con el resto de variables sociodemográficas estudiadas ni con el nivel cognitivo medido por el MEC-30 (ver tabla 67).

Tabla 67: Relación del PDQ-39: Estado cognitivo y las diversas variables.

\begin{tabular}{|c|c|c|}
\hline Variables & $\begin{array}{c}\text { Media } \pm \text { DS/Mediana } \\
(\text { p25-p75) }\end{array}$ & $p$ valor \\
\hline \multicolumn{3}{|l|}{ Sexo } \\
\hline Masculino & $28.24 \pm 18.62$ & \multirow{2}{*}{0.003} \\
\hline Femenino & $41.03 \pm 22.25$ & \\
\hline \multicolumn{3}{|l|}{ Nivel Educativo } \\
\hline Ninguna & $43.75(18.75-81.25)$ & \multirow{4}{*}{0.055} \\
\hline Primaria & $37.50(17.19-50.00)$ & \\
\hline Secundaria & $37.50(12.50-50.00)$ & \\
\hline Superior & $18.75(12.50-34.38)$ & \\
\hline \multicolumn{3}{|l|}{ Estado civil } \\
\hline Casados & $31.25(12.50-46.88)$ & \multirow{2}{*}{0.423} \\
\hline No casados & $21.79(18.75-50.00)$ & \\
\hline \multicolumn{3}{|l|}{ Sector de trabajo } \\
\hline Primario & $31.25(12.50-43.75)$ & \multirow{4}{*}{0.014} \\
\hline Industrial & $37.50(25.00-51.56)$ & \\
\hline Servicios & $18.75(12.50-43.75)$ & \\
\hline Ama de casa & $43.75(31.25-54.69)$ & \\
\hline \multicolumn{3}{|l|}{ Estado cognitivo } \\
\hline Normal & $32.66 \pm 20.91$ & \multirow{3}{*}{0.537} \\
\hline Sospecha de patología & $33.93 \pm 23.90$ & \\
\hline Deterioro & $41.41 \pm 21.11$ & \\
\hline
\end{tabular}


Respecto a la relación de la dimensión estado cognitivo con diversas características de la EP, sólo se encontró asociación con el estadío de Hoehn y Yahr $(\mathrm{p}=0.017)$ (ver tabla 68). La cantidad de tratamientos antiparkinsonianos recibidos no se relacionó con una peor percepción del estado cognitivo (ver tabla 69).

Tabla 68: Relación del PDQ-39: Estado cognitivo y características de la Enfermedad de Parkinson.

\begin{tabular}{lcc}
\hline \hline Variables & $\begin{array}{c}\text { Media } \pm \text { DS } \text { Mediana } \\
(\boldsymbol{p} 25-\boldsymbol{p} 75)\end{array}$ & p valor \\
\hline \hline Antecedente familiar & & \\
$\quad$ No & $35.62 \pm 22.69$ & 0.221 \\
$\quad$ Sí & $29.69 \pm 17.23$ & \\
Edad de inicio (años) & & \\
$<50$ & $15.62(12.50-29.69)$ & 0.061 \\
$\geq 50$ & $37.50(12.50-50.00)$ & \\
Tiempo de evolución (años) & & \\
$<3$ & $27.63 \pm 19.02$ & \\
$\geq 3$ y $<6$ & $36.08 \pm 22.98$ & \\
$\geq 6$ y $\leq 10$ & $29.38 \pm 16.99$ & \\
$>10$ & $40.89 \pm 23.82$ & \\
Estadíos de Hoehn $\boldsymbol{y}$ Yahr & & \\
0 & $12.50(9.38-31.25)$ & \\
1.0 & $25.00(12.50-37.50)$ & \\
1.5 & $43.75(37.50-50.00)$ & \\
2.0 & $12.50(12.50-15.62)$ & 0.017 \\
2.5 & $43.75(20.31-54.69)$ & \\
3.0 & $31.25(17.19-45.31)$ & \\
4.0 & $43.75(37.50-50.00)$ & \\
\hline \hline
\end{tabular}


Tabla 69: Relación del PDQ-39: Estado cognitivo y tratamientos antiparkinsonianos.

\begin{tabular}{lcc}
\hline \hline Variables & Mediana $(p 25-p 75)$ & $p$ valor \\
\hline \hline Tratamiento total & & \\
0 & $28.12(17.19-40.62)$ & \\
1 & $25.00(15.62-43.75)$ & \\
2 & $31.25(12.50-46.88)$ & 0.899 \\
3 & $37.50(20.31-48.44)$ & \\
$\geq 4$ & $18.75(12.50-50.00)$ & \\
Tratamiento oral & & \\
0 & $28.12(17.19-40.62)$ & \\
1 & $25.00(12.50-43.75)$ & \\
2 & $31.25(12.50-50.00)$ & 0.967 \\
3 & $37.50(18.75-43.75)$ & \\
$\geq 4$ & $18.75(12.50-50.00)$ & \\
Terapia avanzada & $33.11 \pm 20.98$ & \\
Ausente & $36.36 \pm 22.33$ & \\
Presente & & \\
\hline \hline
\end{tabular}

La presencia de otras enfermedades médicas no se relacionó con la dimensión estado cognitivo (ver tabla 70). Dentro de los antecedentes psiquiátricos, el haber sido diagnosticado de depresión previo al estudio sí se asoció negativamente con esta dimensión (ver tabla 71). 
Tabla 70: Relación del PDQ-39: Estado cognitivo y otras enfermedades médicas.

\begin{tabular}{|c|c|c|}
\hline Enfermedades médicas & Media $\pm D S$ & p valor \\
\hline \multicolumn{3}{|l|}{ Cardiovascular } \\
\hline Ausente & $33.59 \pm 22.06$ & \multirow{2}{*}{0.953} \\
\hline Presente & $37.50 \pm 19.78$ & \\
\hline \multicolumn{3}{|l|}{ Pulmonar } \\
\hline Ausente & $33.24 \pm 21.16$ & \multirow{2}{*}{0.504} \\
\hline Presente & $38.19 \pm 20.60$ & \\
\hline \multicolumn{3}{|l|}{ Digestivo } \\
\hline Ausente & $33.38 \pm 21.37$ & \multirow{2}{*}{0.813} \\
\hline Presente & $35.94 \pm 12.88$ & \\
\hline \multicolumn{3}{|l|}{ Oncológica } \\
\hline Ausente & $34.20 \pm 21.03$ & \multirow{2}{*}{0.114} \\
\hline Presente & $17.19 \pm 15.62$ & \\
\hline \multicolumn{3}{|l|}{ Otras } \\
\hline Ausente & $28.47 \pm 20.53$ & \multirow{2}{*}{0.144} \\
\hline Presente & $35.48 \pm 21.06$ & \\
\hline
\end{tabular}

Tabla 71: Relación del PDQ-39: Estado cognitivo y antecedente de patología de Salud Mental.

\begin{tabular}{lcc}
\hline \hline Enfermedades psiquiátricas & Media $\pm \boldsymbol{D S}$ & p valor \\
\hline \hline Ansiedad & & \\
Ausente & $33.65 \pm 21.70$ & 0.819 \\
Presente & $31.94 \pm 14.13$ & \\
Depresión & & \\
Ausente & $30.35 \pm 19.70$ & 0.003 \\
Presente & $46.05 \pm 22.07$ & \\
Otra & & \\
Ausente & $33.42 \pm 21.29$ & 0.950 \\
Presente & $33.75 \pm 20.62$ & \\
\hline \hline
\end{tabular}


Se observó una asociación negativa entre toma de antidepresivos y la dimensión de estado cognitivo (ver tabla 72). Así mismo, la presencia de puntuaciones compatibles con depresión y ansiedad en los cuestionarios de Beck-II y STAI-R, respectivamente, evidenciaron un impacto negativo en dicha dimensión (ver tabla 73).

Tabla 72: Relación del PDQ-39: Estado cognitivo y tratamiento antidepresivo y ansiolítico.

\begin{tabular}{ccc}
\hline \hline Tipo de tratamiento & Media $\pm \boldsymbol{D S}$ & $\boldsymbol{p}$ valor \\
\hline \hline & & \\
Tratamiento ansiolítico & & \\
No & $31.14 \pm 20.46$ & 0.175 \\
Sí & $37.16 \pm 21.70$ & \\
Tratamiento antidepresivo & & \\
No & $30.46 \pm 20.10$ & 0.015 \\
Sí & $42.45 \pm 21.65$ & \\
\hline \hline
\end{tabular}

Tabla 73: Relación del PDQ-39: Estado cognitivo y presencia de depresión o ansiedad.

\begin{tabular}{lll}
\hline \hline Variables & Media $\pm \boldsymbol{D S}$ & $\boldsymbol{p}$ valor \\
\hline \hline & & \\
$\boldsymbol{B D I} \boldsymbol{I}-\boldsymbol{I I}$ & & \\
$\quad$ No Depresión & $26.37 \pm 17.97$ & $<0.001$ \\
$\quad$ Depresión & $48.19 \pm 19.45$ & \\
$\boldsymbol{S T A I - R}$ & & \\
$\quad$ No ansiedad & $24.58 \pm 16.97$ & 0.005 \\
Ansiedad & $37.60 \pm 21.58$ & \\
\hline
\end{tabular}




\subsubsection{Calidad de Vida: Comunicación}

El análisis bivariante de la dimensión comunicación con las variables demográficas mostró un impacto negativo del sexo masculino $(\mathrm{p}=0.013)$ y de los pacientes que estaban casados $(\mathrm{p}=0.007)$. No se encontró relación con el estado cognitivo del paciente al momento del estudio (ver tabla 74), con las características generales de la enfermedad de Parkinson (ver tabla 75) ni con el tratamiento antiparkinsoniano recibido (ver tabla 76).

Tabla 74: Relación del PDQ-39: Comunicación y características generales.

\begin{tabular}{lcc}
\hline \hline Variables & $\begin{array}{c}\text { Media } \pm \text { DS } / \\
\text { Mediana }(\text { p25-p75) }\end{array}$ & p valor \\
\hline \hline Sexo & & \\
Masculino & $24.85 \pm 23.00$ & 0.013 \\
$\quad$ Femenino & $13.46 \pm 19.55$ & \\
Nivel Educativo & & \\
$\quad$ Ninguna & $50.00(0.00-66.67)$ & \\
Primaria & $8.33(0.00-33.33)$ & 0.772 \\
Secundaria & $8.33(0.00-33.33)$ & \\
Superior & $16.67(0.00-25.00)$ & \\
Estado civil & & \\
Casados & $16.67(0.00-41.67)$ & 0.007 \\
$\quad$ No casados & $4.17(0.00-10.42)$ & \\
Sector de trabajo & & \\
Primario & $16.67(0.00-50.00)$ & \\
Industrial & $33.33(12.50-43.75)$ & 0.079 \\
Servicios & $8.33(0.00-33.33)$ & \\
Ama de casa & $8.33(0.00-16.67)$ & \\
Estado cognitivo & & \\
Normal & $19.48 \pm 22.26$ & \\
Sospecha de patología & $29.76 \pm 26.73$ & \\
Deterioro & $18.75 \pm 18.77$ & \\
& & \\
\hline \hline
\end{tabular}


Tabla 75: Relación del PDQ-39: Comunicación y características de la Enfermedad de Parkinson.

\begin{tabular}{lcc}
\hline \hline Variables & Mediana (p25-p75) & p valor \\
\hline \hline Antecedente familiar & & \\
$\quad$ No & $16.67(0.00-41.67)$ & 0.213 \\
$\quad$ Sí & $8.33(0.00-27.08)$ & \\
Edad de inicio (años) & $12.50(0.00-16.67)$ & 0.559 \\
$\quad<50$ & $8.33(0.00-33.33)$ & \\
$\geq 50$ & & \\
Tiempo de evolución (años) & $0.00(0.00-25.00)$ & \\
$<3$ & $12.50(0.00-31.25)$ & 0.064 \\
$\geq 3$ y $<6$ & $8.33(2.08-29.16)$ & \\
$\geq 6$ y $\leq 10$ & $20.84(8.33-43.75)$ & \\
$>10$ & & \\
Estadíos de Hoehn y Yahr & $8.33(4.17-45.83)$ & \\
0 & $0.00(0.00-16.67)$ & \\
1.0 & $8.33(0.00-33.33)$ & \\
1.5 & $0.00(0.00-25.00)$ & 0.064 \\
2.0 & $16.67(8.33-31.25)$ & \\
2.5 & $16.67(8.33-41.67)$ \\
3.0 & $33.33(8.33-41.67)$ \\
4.0 & & \\
& & \\
\hline \hline
\end{tabular}


Tabla 76: Relación del PDQ-39: Comunicación y tratamientos antiparkinsonianos.

\begin{tabular}{ccc}
\hline \hline Variables & $\begin{array}{c}\text { Media } \pm \text { DS/ Mediana } \\
(\boldsymbol{p} 25-\boldsymbol{p} 75)\end{array}$ & $\boldsymbol{p}$ valor \\
\hline \hline Tratamiento total & & \\
0 & $54.16(31.25-77.08)$ & \\
1 & $0.00(0.00-33.33)$ & \\
2 & $8.33(0.00-16.67)$ & 0.097 \\
3 & $16.67(0.00-39.59)$ & \\
$\geq 4$ & $8.33(4.17-33.34)$ & \\
Tratamiento oral & & \\
0 & $54.16(31.25-77.08)$ & \\
1 & $16.67(0.00-33.33)$ & \\
2 & $8.33(0.00-27.08)$ & 0.151 \\
3 & $8.33(0.00-33.33)$ & \\
$\geq 4$ & $8.33(8.33-50)$ & \\
Terapia avanzada & & \\
Ausente & $19.15 \pm 22.06$ & 0.215 \\
Presente & $28.03 \pm 23.36$ & \\
\hline \hline
\end{tabular}

Se buscó si existía relación con otras enfermedades médicas o historia de patología psiquiátrica previa, no encontrándose asociación con la dimensión de comunicación (ver tablas 77 y 78). Tampoco se encontró asociación con la toma de ansiolíticos o de tratamiento antidepresivo (ver tabla 79). 
Tabla 77: Relación del PDQ-39: Comunicación y otras enfermedades médicas.

\begin{tabular}{lcc}
\hline \hline Enfermedades médicas & $\begin{array}{c}\text { Media } \pm \text { DS/Mediana } \\
(\boldsymbol{p} 25-\boldsymbol{p} \text { 75) }\end{array}$ & p valor \\
\hline \hline Cardiovascular & & \\
Ausente & $19.94 \pm 21.54$ & 0.903 \\
Presente & $20.51 \pm 23.56$ & \\
Pulmonar & & \\
Ausente & $19.31 \pm 22.09$ & 0.151 \\
Presente & $30.55 \pm 22.82$ & \\
Digestivo & & \\
Ausente & & \\
Presente & $20.42 \pm 22.58$ & \\
Oncológica & $14.58 \pm 14.23$ & \\
$\quad$ Ausente & & \\
Presente & $20.60 \pm 22.48$ & \\
Otras & $10.41 \pm 15.77$ & \\
Ausente & & \\
Presente & $8.33(0.00-16.67)$ & \\
\hline \hline
\end{tabular}

Tabla 78: Relación del PDQ-39: Comunicación y antecedente de patología de Salud Mental.

\begin{tabular}{lcc}
\hline \hline Enfermedades psiquiátricas & $\begin{array}{c}\text { Media } \pm \text { DS/Mediana } \\
(\boldsymbol{p} 25-\boldsymbol{p} 75)\end{array}$ & p valor \\
\hline \hline Ansiedad & & \\
$\quad$ Ausente & $20.25 \pm 22.53$ & 0.918 \\
$\quad$ Presente & $19.44 \pm 20.83$ & \\
Depresión & & \\
$\quad$ Ausente & $8.33(0.00-33.33)$ & 0.081 \\
$\quad$ Presente & $16.67(8.33-41.66)$ & \\
Otra & & \\
$\quad$ Ausente & $8.33(0.00-33.33)$ & 0.660 \\
Presente & $16.67(0.00-33.33)$ & \\
& & \\
\hline \hline
\end{tabular}


Tabla 79: Relación del PDQ-39: Comunicación y tratamiento antidepresivo y ansiolítico.

\begin{tabular}{ccc}
\hline \hline Tipo de tratamiento & $\begin{array}{c}\text { Media } \pm \text { DS/Mediana } \\
(\boldsymbol{p} 25-\boldsymbol{p} 75)\end{array}$ & p valor \\
\hline \hline & & \\
Tratamiento ansiolítico & $18.53 \pm 22.84$ & \\
No & $22.75 \pm 21.40$ & 0.371 \\
Sí & & \\
Tratamiento antidepresivo & $8.33(0.00-33.33)$ & 0.648 \\
No & $12.50(6.25-33.33)$ & \\
Sí & & \\
\end{tabular}

La presencia de puntuaciones positivas para depresión en el Beck-II mostró una asociación estadísticamente significativa con una peor percepción de la dimensión comunicación de la calidad de vida en los pacientes $(\mathrm{p}<0.001)$. Dicha asociación también se evidenció en los pacientes que cumplieron los criterios de ansiedad según el STAI-R $(\mathrm{p}=0.017)($ ver tabla 80$)$.

Tabla 80: Relación del PDQ-39: Comunicación y presencia de depresión o ansiedad.

\begin{tabular}{lcc}
\hline \hline Variables & Media $\pm \boldsymbol{D S}$ & $\boldsymbol{p}$ valor \\
\hline \hline & & \\
$\boldsymbol{B D I} \boldsymbol{I} \boldsymbol{I I}$ & & \\
$\quad$ No Depresión & $14.71 \pm 19.46$ & $<0.001$ \\
$\quad$ Depresión & $31.45 \pm 23.74$ & \\
$\boldsymbol{S T} \boldsymbol{A I} \boldsymbol{R}$ & & \\
$\quad$ No ansiedad & $12.22 \pm 17.33$ & 0.017 \\
Ansiedad & $23.85 \pm 23.43$ & \\
\hline \hline
\end{tabular}




\subsubsection{Calidad de Vida: Dolor}

Respecto a la relación de las variables sociodemográficas con la dimensión dolor, en el análisis bivariante se encontró que pertenecer al sexo femenino se asociaba a una peor percepción de esta dimensión $(\mathrm{p}=0.002)$. No se encontró asociación con el nivel educativo, el estado civil ni el sector en el que trabajan los pacientes. Así mismo, los pacientes que presentaban una sospecha de deterioro cognitivo según las puntuaciones del MEC-30, también se asociaban a un deterioro de la dimensión dolor $(\mathrm{p}=0.020)$ (ver tabla 81).

Tabla 81: Relación del PDQ-39: Dolor y características generales.

\begin{tabular}{lcc}
\hline \hline Variables & $\begin{array}{c}\text { Media } \pm \text { DS/ Mediana } \\
(\boldsymbol{p} 25-\boldsymbol{p} 75)\end{array}$ & $\boldsymbol{p}$ valor \\
\hline \hline Sexo & & \\
Masculino & $30.80 \pm 22.75$ & 0.002 \\
$\quad$ Femenino & $45.30 \pm 21.27$ & \\
Nivel Educativo & & \\
Ninguna & $41.67 \pm 31.18$ & \\
Primaria & $33.54 \pm 24.20$ & \\
Secundaria & $45.06 \pm 21.09$ & \\
Superior & $31.52 \pm 20.41$ & \\
Estado civil & & \\
Casados & $35.20 \pm 23.87$ & \\
$\quad$ No casados & $40.48 \pm 21.36$ & \\
Sector de trabajo & & \\
Primario & & \\
Industrial & $33.33(16.67-62.50)$ & \\
Servicios & $20.84(6.25-50.00)$ & \\
Ama de casa & $33.33(25.00-50.00$ & \\
Estado cognitivo & $50.00(33.33-58.33)$ & \\
Normal & & \\
Sospecha de patología & $59.52 \pm 29.83$ & \\
Deterioro & $39.59 \pm 26.26$ & \\
\hline \hline
\end{tabular}


De las diversas características de la EP estudiadas, se encontró que el no tener el antecedente familiar de EP tenía un impacto negativo en la dimensión dolor cuando se lo comparaba con aquellos pacientes que sí tenían algún familiar con esta patología ( $\mathrm{p}=$ 0.028). No se encontró relación con la edad de inicio, el tiempo de evolución, el estadío de Hoehn y Yahr (ver tabla 82), ni con el tratamiento antiparkinsoniano recibido (ver tabla 83).

Tabla 82: Relación del PDQ-39: Dolor y características de la Enfermedad de Parkinson.

\begin{tabular}{lll}
\hline \hline Variables & Media $\pm \boldsymbol{D S}$ & p valor \\
\hline \hline Antecedente familiar & & \\
$\quad$ No & $40.87 \pm 23.98$ & 0.028 \\
$\quad$ Sí & $29.17 \pm 20.73$ & \\
Edad de inicio (años) & & \\
$\quad<50$ & $43.33 \pm 28.54$ & 0.345 \\
$\geq 50$ & $35.98 \pm 22.54$ & \\
Tiempo de evolución (años) & & \\
$<3$ & $25.88 \pm 20.20$ & \\
$\geq 3$ y $<6$ & $40.15 \pm 26.05$ & \\
$\geq 6$ y $\leq 10$ & $39.44 \pm 17.63$ & \\
$>10$ & $38.89 \pm 27.22$ & \\
Estadíos de Hoehn $\boldsymbol{y}$ Yahr & & \\
0 & $36.11 \pm 55.49$ & \\
1.0 & $31.67 \pm 18.00$ & \\
1.5 & $40.74 \pm 20.60$ & \\
2.0 & $25.00 \pm 15.96$ & \\
2.5 & $37.50 \pm 25.89$ & \\
3.0 & $41.67 \pm 26.01$ & \\
4.0 & $40.39 \pm 20.08$ & \\
\hline \hline
\end{tabular}


Tabla 83: Relación del PDQ-39: Dolor y tratamientos antiparkinsonianos.

\begin{tabular}{lll}
\hline \hline Variables & Media $\pm \boldsymbol{D S}$ & $\boldsymbol{p}$ valor \\
\hline \hline Tratamiento total & & \\
0 & $60.42 \pm 29.95$ & \\
1 & $29.45 \pm 21.33$ & \\
2 & $35.49 \pm 21.01$ & 0.156 \\
3 & $39.71 \pm 24.28$ & \\
$\geq 4$ & $33.33 \pm 22.27$ & \\
Tratamiento oral & & \\
0 & $60.42 \pm 29.95$ & \\
1 & $32.35 \pm 22.02$ & \\
2 & $34.64 \pm 21.80$ & \\
3 & $39.65 \pm 24.67$ & \\
$\geq 4$ & $33.97 \pm 20.82$ & \\
Terapia avanzada & & \\
Ausente & $36.51 \pm 22.88$ & \\
Presente & $38.64 \pm 26.43$ & \\
\hline \hline
\end{tabular}

No se encontró un impacto negativo de otras enfermedades médicas ni de la historia de patología de salud mental previa, ni de la toma de ansiolíticos o antidepresivos en la dimensión dolor (ver tablas 84,85 y 86). 
Tabla 84: Relación del PDQ-39: Dolor y otras enfermedades médicas.

\begin{tabular}{lll}
\hline \hline & & \\
Enfermedades médicas & Media $\pm \boldsymbol{D S}$ & \\
\hline \hline Cardiovascular & & \\
$\quad$ Ausente & $33.18 \pm 22.78$ & 0.072 \\
Presente & $41.88 \pm 23.06$ & \\
Pulmonar & & \\
$\quad$ Ausente & $37.26 \pm 23.84$ & 0.633 \\
Presente & $33.33 \pm 17.68$ & \\
Digestivo & & \\
$\quad \begin{array}{l}\text { Ausente } \\
\text { Presente }\end{array}$ & $36.72 \pm 23.54$ & \\
Oncológica & $37.50 \pm 14.43$ & \\
$\quad$ Ausente & & \\
Presente & $37.00 \pm 23.61$ & \\
Otras & $31.25 \pm 7.98$ & \\
$\quad$ Ausente & & \\
Presente & $34.57 \pm 19.98$ & \\
\hline \hline
\end{tabular}

Tabla 85: Relación del PDQ-39: Dolor y antecedente de patología de Salud Mental.

$\begin{array}{ccc}\text { Enfermedades psiquiátricas } & \text { Media } \pm \text { DS/Mediana } & \text { p valor }\end{array}$

\begin{tabular}{ccc}
\hline \hline Ansiedad & & \\
Ausente & $36.24 \pm 23.63$ & 0.507 \\
Presente & $41.67 \pm 18.63$ & \\
Depresión & & \\
Ausente & $33.33(16.67-50.00)$ & 0.088 \\
Presente & $50.00(33.33-58.33)$ & \\
Otra & $36.22 \pm 22.78$ & \\
Ausente & $38.75 \pm 25.11$ & 0.667 \\
Presente & \\
\hline \hline
\end{tabular}


Tabla 86: Relación del PDQ-39: Dolor y tratamiento antidepresivo y ansiolítico.

\begin{tabular}{ccc}
\hline \hline Tipo de tratamiento & $\begin{array}{c}\text { Media } \pm \text { SD/ Mediana } \\
(\mathbf{p} 25-\mathbf{p} 75)\end{array}$ & p valor \\
\hline \hline & & \\
Tratamiento ansiolítico & $35.92 \pm 22.09$ & 0.662 \\
No & $38.06 \pm 25.04$ & \\
Sí & & 0.065 \\
Tratamiento antidepresivo & $33.33(16.67-50.00)$ & \\
No & $50.00(31.25-58.33)$ & \\
Sí & & \\
\hline \hline
\end{tabular}

La presencia de sintomatología depresiva, medida por el BDI-II, y de sintomatología ansiosa, medida por el STAI-R, influyeron negativamente en la dimensión dolor (ver tabla 87).

Tabla 87: Relación del PDQ-39: Dolor y presencia de depresión o ansiedad.

\begin{tabular}{lcc}
\hline \hline Variables & Media $\pm \boldsymbol{D S}$ & $\boldsymbol{p}$ valor \\
\hline \hline & & \\
$\boldsymbol{B D I}$-II & & \\
$\quad$ No Depresión & $33.33 \pm 22.27$ & 0.038 \\
$\quad$ Depresión & $43.82 \pm 23.76$ & \\
$\boldsymbol{S T A I - R}$ & & 0.002 \\
$\quad$ No ansiedad & $26.11 \pm 19.78$ & \\
$\quad$ Ansiedad & $41.67 \pm 23.11$ & \\
\hline \hline
\end{tabular}




\subsubsection{Calidad de Vida: PDSI}

La calidad de vida global medida por el PDSI, no se relacionó con los aspectos sociodemográficos estudiados ni con el estado cognitivo (ver tabla 88).

Tabla 88: Relación del PDQ-39: PDSI y características generales.

\begin{tabular}{lcc}
\hline \hline Variables & $\begin{array}{c}\text { Media } \pm \text { DS } / \\
\text { Mediana }(\boldsymbol{p} 25-\boldsymbol{p} 75)\end{array}$ & p valor \\
\hline \hline Sexo & & \\
Masculino & $25.06 \pm 12.94$ & 0.231 \\
Femenino & $28.49 \pm 14.53$ & \\
Nivel Educativo & & \\
Ninguna & $34.32(10.68-60.52)$ & \\
Primaria & $25.26(19.27-36.07)$ & \\
Secundaria & $27.14(20.57-34.46)$ & \\
Superior & $23.75(14.56-29.64)$ & \\
Estado civil & & \\
Casados & $25.83(16.82-34.40)$ & \\
$\quad$ No casados & $24.17(18.03-34.39)$ & \\
Sector de trabajo & & \\
Primario & $25.35 \pm 12.69$ & \\
Industrial & $29.59 \pm 15.12$ & \\
Servicios & $23.95 \pm 13.70$ & \\
Ama de casa & $30.10 \pm 11.77$ & \\
Estado cognitivo & & \\
Normal & $25.42 \pm 13.09$ & \\
Sospecha de patología & $32.67 \pm 16.71$ & \\
Deterioro & $31.52 \pm 15.66$ & \\
\hline \hline
\end{tabular}


En la tabla 89 se aprecia la relación del PDSI con características generales de la EP. Se encontró relación entre un mayor tiempo de evolución de enfermedad y un mayor estadío de Hoehn y Yahr con una peor calidad de vida ( $\mathrm{p}=0.035$ y $\mathrm{p}=0.001$ respectivamente). No se encontró relación con el antecedente familiar, ni la edad de inicio de la EP (ver tabla 89). Tampoco se encontró asociación con el tratamiento antiparkinsoniano recibido (ver tabla 90).

Tabla 89: Relación del PDQ-39: PDSI y características de la Enfermedad de Parkinson.

\begin{tabular}{lcc}
\hline \multicolumn{1}{c}{ Variables } & Media \pm DS & p valor \\
\hline \hline Antecedente familiar & & \\
$\quad$ No & $28.60 \pm 14.20$ & 0.052 \\
$\quad$ Sí & $22.55 \pm 11.72$ & \\
Edad de inicio (años) & & \\
$\quad<50$ & $22.01 \pm 13.89$ & 0.277 \\
$\geq 50$ & $26.99 \pm 13.60$ & \\
Tiempo de evolución (años) & & \\
$<3$ & $21.04 \pm 12.67$ & \\
$\geq 3$ y $<6$ & $28.33 \pm 10.87$ & \\
$\geq 6$ y $\leq 10$ & $24.04 \pm 11.52$ & \\
$>10$ & $32.09 \pm 17.08$ & \\
Estadíos de Hoehn $\boldsymbol{y}$ Yahr & & \\
0 & $21.56 \pm 23.38$ & \\
1.0 & $20.17 \pm 10.83$ & \\
1.5 & $32.79 \pm 18.96$ & \\
2.0 & $15.01 \pm 9.22$ & \\
2.5 & $28.38 \pm 9.48$ & \\
3.0 & $28.11 \pm 12.26$ & \\
4.0 & $36.42 \pm 12.34$ & \\
& & \\
\hline \hline
\end{tabular}


Tabla 90: Relación del PDQ-39: PDSI y tratamientos antiparkinsonianos.

\begin{tabular}{lll}
\hline \hline Variables & Media $\pm \boldsymbol{D S}$ & $\boldsymbol{p}$ valor \\
\hline \hline Tratamiento total & & \\
0 & $35.07 \pm 10.41$ & \\
1 & $29.32 \pm 18.38$ & \\
2 & $24.15 \pm 14.04$ & 0.546 \\
3 & $26.52 \pm 11.45$ & \\
$\geq 4$ & $25.38 \pm 13.13$ & \\
Tratamiento oral & & \\
0 & $35.07 \pm 10.41$ & \\
1 & $30.03 \pm 18.19$ & \\
2 & $14.90 \pm 13.23$ & \\
3 & $25.40 \pm 11.02$ & \\
$\geq 4$ & $25.42 \pm 14.18$ & \\
Terapia avanzada & & \\
Ausente & $25.93 \pm 13.85$ & \\
Presente & $30.56 \pm 11.70$ & \\
& & \\
\hline \hline
\end{tabular}


Al analizar si la presencia de otras enfermedades médicas se relacionaba con un empeoramiento de la calidad de vida, no se encontró ninguna relación (ver tabla 91). Sí se encontró asociación entre una peor calidad de vida y el haber sido diagnosticado de depresión previo al estudio $(p=0.026)$ y estar en tratamiento antidepresivo $(p=0.017)$ (ver tablas 92 y 93).

Tabla 91: Relación del PDQ-39: PDSI y otras enfermedades médicas.

\begin{tabular}{llc}
\hline \hline Enfermedades médicas & Media $\pm \boldsymbol{D S}$ & p valor \\
\hline \hline $\begin{array}{l}\text { Cardiovascular } \\
\text { Ausente } \\
\text { Presente }\end{array}$ & $25.11 \pm 14.48$ & \\
Pulmonar & $28.42 \pm 12.26$ & 0.246 \\
$\quad$ Ausente & & \\
Presente & $26.38 \pm 13.89$ & 0.524 \\
Digestivo & $29.43 \pm 10.21$ & \\
$\quad \begin{array}{l}\text { Ausente } \\
\text { Presente }\end{array}$ & $26.51 \pm 13.62$ & \\
Oncológica & $25.45 \pm 16.21$ & \\
$\quad \begin{array}{l}\text { Ausente } \\
\text { Presente }\end{array}$ & & \\
Otras & $26.89 \pm 13.61$ & \\
$\quad$ Ausente & $16.83 \pm 12.19$ & \\
Presente & & \\
\hline \hline
\end{tabular}


Tabla 92: Relación del PDQ-39: PDSI y antecedente de patología de Salud Mental.

\begin{tabular}{lcc}
\hline \hline Enfermedades psiquiátricas & Media $\pm \boldsymbol{D S}$ & p valor \\
\hline \hline $\begin{array}{l}\text { Ansiedad } \\
\text { Ausente } \\
\text { Presente }\end{array}$ & $26.31 \pm 14.13$ & \\
Depresión \\
$\quad \begin{array}{l}\text { Ausente } \\
\text { Presente }\end{array}$ & $27.94 \pm 7.85$ & 0.735 \\
Otra & & \\
$\quad$ Ausente & $24.92 \pm 13.66$ & 0.026 \\
Presente & $36.66 \pm 11.96$ & \\
& $26.14 \pm 13.35$ & 0.658 \\
\hline \hline
\end{tabular}

Tabla 93: Relación del PDQ-39: PDSI y tratamiento antidepresivo y ansiolítico.

\begin{tabular}{ccc}
\hline \hline Tipo de tratamiento & Media \pm DS & p valor \\
\hline \hline & & \\
Tratamiento ansiolítico & & \\
No & $25.16 \pm 13.12$ & 0.244 \\
Sí & $28.52 \pm 14.36$ & \\
Tratamiento antidepresivo & & 0.017 \\
No & $24.54 \pm 13.83$ & \\
Sí & $32.17 \pm 11.54$ & \\
\hline \hline
\end{tabular}


La presencia de sintomatología depresiva medida por el BDI-II y de sintomatología ansiosa medida por el STAI-R nos muestra que la presencia de dichos síntomas influyen negativamente en la calidad de vida (ver tabla 94).

Tabla 94: Relación del PDQ-39: PDSI y presencia de depresión o ansiedad.

\begin{tabular}{|c|c|c|}
\hline Variables & Media $\pm D S$ & $p$ valor \\
\hline \multicolumn{3}{|l|}{ BDI-II } \\
\hline No Depresión & $21.00 \pm 9.77$ & $<0.001$ \\
\hline Depresión & $37.76 \pm 13.69$ & \\
\hline \multicolumn{3}{|l|}{$S T A I-R$} \\
\hline No ansiedad & $17.34 \pm 9.41$ & $<0.001$ \\
\hline Ansiedad & $30.68 \pm 13.27$ & \\
\hline
\end{tabular}

\subsubsection{Calidad de Vida: Regresión lineal múltiple}

La tabla 95 muestra la regresión lineal múltiple de las diversas dimensiones del PDQ39 y el PDSI con las diversas variables. Todas las dimensiones, excepto las de apoyo social y dolor, se asociaron con la presencia de depresión $(\mathrm{p}<0.005)$. La ansiedad fue también una variable determinante significativa del PDSI $(\mathrm{p}=0.015)$ y se asoció a las dimensiones bienestar emocional $(\mathrm{p}<0.001)$ y dolor $(\mathrm{p}=0.014)$.

Con respecto al sexo, ser del sexo masculino tuvo una asociación positiva con las dimensiones de bienestar emocional $(p=0.006)$, estado cognitivo $(p=0.003)$ y dolor $(\mathrm{p}=0.001)$ y una asociación negativa con la dimensión comunicación $(\mathrm{p}=0.001)$.

Se encontró una relación estadísticamente significativa de la afectación motora con las dimensiones de movilidad (estadío 4.0 de Hoenh y Yahr: $\mathrm{p}=0.004$ ), actividades de la vida diaria $(\mathrm{p}=0.027$ y $\mathrm{p}=0.001$ : estadíos 3.0 y 4.0 respectivamente), bienestar emocional (estadío 1.0: $\mathrm{p}=0.006$; estadío 1.5: $\mathrm{p}=0.001$; estadío 2.0: $\mathrm{p}=0,032$; estadío 2.5: $\mathrm{p}<0.001 ;$ estadío $3.0 \mathrm{p}=0.012$ y estadío $4.0: \mathrm{p}=0.001)$ y estado cognitivo $(\mathrm{p}=0.023$ y $\mathrm{p}=0.03$ : estadíos 2.5 y 4.0 respectivamente). 
La dimensión de apoyo social se asoció significativamente solamente con aquellos que presentaron un inicio de síntomas a la edad de 50 años o más $(\mathrm{p}=0,030)$. En este caso el R2 ajustado fue bastante bajo $(0,0506)$.

Entre las patologías crónicas, la enfermedad cardiovascular y la patología pulmonar se asociaron con una peor valoración de las dimensiones de movilidad y actividades de la vida diaria respectivamente $(\mathrm{p}=0.038$ y $\mathrm{p}=0.003)$.

Tener un nivel educativo básico (primaria) y el uso de más un tratamiento antiparkinsoniano tuvo un impacto positivo en la dimensión de actividades de la vida diaria $(\mathrm{p}=0.048$ y $\mathrm{p}=0.025$ respectivamente).

El modelo mostró un $\mathrm{R}^{2}$ ajustado de 0,4908 para la calidad de vida relacionada con la salud medida por el PDSI. Este análisis muestra una influencia significativa de las manifestaciones motoras de la EP, medidas por el estadío de Hoehn y Yahr ( $\mathrm{p}=0.013$ en el estadío 4); pacientes con depresión, según puntuaciones del BDI-II $(\mathrm{p}<0.001)$; $\mathrm{y}$ ansiedad, según las puntuaciones del STAI-R ( $<<0.015)$.

Además, se realizó la correlación parcial al cuadrado para ver la contribución de las principales variables consideradas en el modelo de la calidad de vida relacionada con la salud. Se encontró que la variable que más influía fue la depresión (23.30\%), seguida inmediatamente por el estadío de Hoehn y Yarh (23.02\%). La ansiedad influyó con el $8.24 \%$ de la variabilidad del modelo. 
Tabla 95: Análisis de regresión lineal de la calidad de vida y otras variables.

\begin{tabular}{|c|c|c|c|c|c|c|c|c|c|}
\hline Variables & Movilidad & $A V D$ & $B E$ & Estigma & $\begin{array}{r}\text { Apoyo } \\
\text { social } \\
\end{array}$ & $\begin{array}{c}\text { Estado } \\
\text { cognitivo }\end{array}$ & Comunica & Dolor & PDSI \\
\hline Sexo masculino & - & - & $-9.501 * *$ & - & - & $-14.690 * *$ & $14.708^{* *}$ & $-15.935 * *$ & - \\
\hline \multicolumn{10}{|l|}{ Nivel educativo } \\
\hline Primaria & - & $-19.916^{*}$ & -2.724 & - & - & -10.254 & - & - & -5.500 \\
\hline Secundaria & - & -10.033 & 6.384 & - & - & -3.862 & - & - & -0.308 \\
\hline Superior & - & -19.108 & 2.349 & - & - & -13.301 & - & - & -7.136 \\
\hline \multicolumn{10}{|l|}{ Sector de trabajo } \\
\hline Industrial & - & - & - & - & - & 4.320 & - & - & - \\
\hline Servicios & - & - & - & - & - & -9.198 & - & - & - \\
\hline Ama de casa & - & - & - & - & - & -3.394 & - & - & - \\
\hline Antecedente familiar de EP & - & - & - & - & - & - & - & -9.209 & - \\
\hline Edad de inicio $(\geq 50$ vears) & - & - & - & - & $16.462 *$ & - & - & - & - \\
\hline \multicolumn{10}{|l|}{ Estadíos de Hoehn y Yahr } \\
\hline Estadío 1.0 & 2.191 & 12.799 & $24.395 * *$ & - & - & 10.547 & - & - & 2.843 \\
\hline Estadío 1.5 & 15.799 & 16.987 & $31.801 * *$ & - & - & 20.986 & - & - & 9.914 \\
\hline Estadío 2.0 & -5.802 & 8.78 & $21.462 *$ & - & - & 1.416 & - & - & -2.19 \\
\hline Estadío 2.5 & 6.316 & 18.889 & $35.049 * *$ & - & - & $24.262^{*}$ & - & - & 10.307 \\
\hline Estadío 3.0 & 8.994 & $27.755^{*}$ & $22.300 *$ & - & - & 11.798 & - & - & 6.782 \\
\hline Estadío 4.0 & $35.241 * *$ & $43.954 * *$ & $31.939 * *$ & - & - & $23.514^{*}$ & - & - & $16.218^{*}$ \\
\hline \multicolumn{10}{|l|}{ Estado cognitivo } \\
\hline Sospecha patología & - & - & - & - & - & - & - & $23.930 * *$ & - \\
\hline Deterioro & - & - & - & - & - & - & - & 2.019 & - \\
\hline $\begin{array}{l}\text { >1 Tto. antiparkinsoniano } \\
\text { Otras enfermedades }\end{array}$ & - & $-12.702 *$ & - & 6.355 & - & - & - & - & - \\
\hline Cardiovascular & $8.399 *$ & - & - & - & - & - & - & - & - \\
\hline Pulmonar & - & $21.810 * *$ & - & - & - & - & - & - & - \\
\hline Tratamiento antidepresivo & - & - & 6.169 & - & - & - & - & - & - \\
\hline BDI-II & $12.204 * *$ & $15.856 * *$ & $16.812 * *$ & $8.318 * *$ & 6.946 & $14.234 * *$ & $18.374 * *$ & -0.926 & $12.423 * *$ \\
\hline STAI-R & 7.976 & 2.791 & $14.011 * *$ & 4.851 & - & 5.622 & 3.266 & $13.017 *$ & $6.019 *$ \\
\hline Adjusted $R^{2}$ & 0.3982 & 0.4005 & 0.55 & 0.1307 & 0.0506 & 0.432 & 0.212 & 0.2524 & 0.4908 \\
\hline
\end{tabular}

$* \mathrm{p}<0.05 ; * * \mathrm{p}<0.01$. AVL: Actividades de la vida diaria. BE: Bienestar emocional. Estigma: Estigmatización. Comunica: Comunicación 
RISCCUSIÓN 


\section{DISCUSIÓN}

En los últimos años se han publicado varios trabajos analizando los trastornos neuropsiquiátricos en la Enfermedad de Parkinson. En este contexto, varios estudios coinciden en señalar a la depresión y a la ansiedad entre los más prevalentes. ${ }^{(69-71)}$. El presente estudio encontró una elevada prevalencia de estas patologías, superiores a la población general, en la población estudiada. Sin embargo, aunque en muchos estudios se encontró la depresión como el trastorno neuropsiquiátrico más frecuente ${ }^{(72,73)}$, en este estudio se encontró una mayor prevalencia de ansiedad. Este resultado concuerda con en un estudio realizado sobre la base DoPaMiP de Francia, ${ }^{(74)}$ que encontró una prevalencia de ansiedad (51\% de ansiedad $\mathrm{v} / \mathrm{s} 41 \%$ de depresión) y con otro estudio realizado en China, ${ }^{(75)}$ aunque en éste último caso las cifras de prevalencia fueron inferiores $(25.81 \%$ $\mathrm{v} / \mathrm{s} 11.17 \%$ ). Esta variación de los resultados en los diversos estudios podría deberse a los diferentes criterios metodológicos así como a la aplicación de diferentes escalas diagnósticas y puntos de corte.

Se encontró una elevada coexistencia de ansiedad y depresión, encontrándose además que entre los pacientes que tenían depresión, la mayor parte también tenía niveles de ansiedad elevados (77.42\% de STAI/E y $96.77 \%$ de STAI/R). Resultados similares se encontraron en otros estudios, incluso cuando fueron comparados con otras enfermedades crónicas (71,74,75). Estos datos sugieren que la génesis de la enfermedad de Parkinson, la ansiedad y la depresión estarían relacionadas y que probablemente tienen una alteración neurobiológica común. Como es sabido, en la enfermedad de Parkinson existe una alteración de los neurotransmisores y pérdida neuronal en el área tegmental ventral y en el locus coeruleus que influirían en la aparición de estos síntomas ${ }^{(32)}$.

La relación de la depresión y la ansiedad con otras variables sociodemográficas difiere en los diferentes estudios ${ }^{(70,75-78)}$. En este estudio, aunque se encontraron puntuaciones más altas del BDI-II en mujeres y del STAI/R en varones, no se encontró una asociación estadísticamente significativa, lo que contrasta con otros estudios en los que se encontraron una relación significativa de ambas patologías con el sexo femenino ${ }^{(31,75-79)}$. 
Este estudio muestra asociación de la depresión con el tiempo de evolución de la EP pero no con su gravedad (medida por los estadíos de Hoehn y Yahr). Esto coincide parcialmente con estudios previos que encontraron que el aumento de ambas variables aumenta a su vez el riesgo de padecer depresión ${ }^{(26,71,74,75)}$.

Por otro lado, la relación de la ansiedad con el tiempo de evolución y la gravedad de la EP difieren de un estudio a otro ${ }^{(31,37,70,75,78,79)}$. En el presente trabajo no se encontró asociación con ninguna de esas variables, lo que coincide con los resultados del estudio multicéntrico realizado por Starkstein et al. ${ }^{(31)}$ y un estudio francés realizado sobre la base $\mathrm{DoPaMiP}{ }^{(74)}$, que no encontraron relación de la ansiedad con el tiempo de evolución ni con los estadíos de Hoehn y Yahr.

A diferencia de otros estudios ${ }^{(74,80,81,82)}$, aunque se encontró mayor prevalencia de deterioro cognitivo en los pacientes con depresión, esta no fue significativa. Por otro lado, en la ansiedad los estudios tienen resultados contradictorios; así mientras algunos no encontraron relación con el deterioro cognitivo ${ }^{(31,71,74,84)}$, otros sí lo hicieron ${ }^{(78,79)}$. En este estudio se vio que aquellos pacientes que tenían puntuaciones del MEC-30 discretamente inferiores a las esperadas para su edad y grado de instrucción (sospecha de deterioro), tenían más riesgo de ansiedad, no encontrándose esta relación en los que ya presentaban un deterioro cognitivo marcado.

Otro aspecto que se analizó fue la asociación con los tratamientos recibidos. Los pacientes con enfermedad de Parkinson presentan fluctuaciones en el estado de ánimo y en los niveles de ansiedad que se cree podrían estar en relación con los períodos "on-off" secundarios al tratamiento antiparkinsoniano, especialmente de L-Dopa ${ }^{(84,85)}$. En este estudio no se encontró esta relación con ninguno de los síntomas estudiados. Esto podría deberse a que se consideró el tratamiento antiparkinsoniano por grupos pero no por la dosis recibida.

La calidad de vida relacionada con la salud en la EP es otro aspecto que se ha empezado a estudiar, ya que proporciona información importante para evaluar la eficacia del tratamiento ${ }^{\left({ }^{8}\right)}$. En el presente estudio, al realizar el análisis multivariado, no se encontró 
relación de la CVRS medido por el PDSI, con la edad, el estado civil o el antecedente familiar de EP. Tampoco se encontró relación de dichas variables con ninguna de las dimensiones del PDQ-39.

La relación del género y la CVRS difieren en los diversos estudios ${ }^{(87-90)}$. Ophey et al estudiaron en Alemania a 245 pacientes, encontrando que el ser mujer era un predictor negativo para las dimensiones que medían el funcionamiento físico y emocional de la calidad de vida, mientras que el ser varón era un predictor negativo para las de la cognición. El presente estudio encontró peor calidad de vida en los varones en la dimensión de comunicación y; peor calidad de vida en las dimensiones de bienestar emocional, dolor y estado cognitivo en las mujeres. No se encontró influencia del género en el PDSI. La diferencia de resultados en los diversos estudios se podría explicar por la complejidad de la CVRS. Además, la diferencia de percepción entre varones y mujeres podría deberse a una influencia hormonal, cultural y social. Moore et al. estudiaron la relación entre la identidad de género y la calidad de vida, encontrando que los varones y mujeres con características andróginas (aquellos/(as) con un elevado número de características masculinas y femeninas) presentaban una mejor calidad de vida, siendo esto más notorio en las mujeres andróginas ${ }^{(92)}$.

Algunos estudios indican que un mayor nivel educativo tiene efectos protectores en la función cognitiva y, secundariamente, influiría positivamente en la calidad de vida ${ }^{(93,94)}$. El presente estudio encontró que aquellas personas que tenían una educación primaria presentaban menor riesgo de deterioro de la dimensión de actividades de la vida diaria pero no de la dimensión de estado cognitivo. Además, se encontró asociación entre los pacientes que presentaban un mínimo deterioro en el MEC-30 (sospecha de deterioro cognitivo) y la dimensión de dolor pero no con el PDSI ni con la dimensión estado cognitivo del PDQ-39. Esto último podría ser explicado porque, como se muestra en el estudio de Jones et al., la dimensión del estado cognitivo del PDQ-39 no presenta una relación fuerte con los tests neurocognitivos pero sí con la presencia de depresión ${ }^{(95)}$, cuya prevalencia fue elevada en el presente estudio e influyó significativamente en esa dimensión. Este hallazgo refuerza la idea de que las quejas cognitivas en los pacientes con EP podrían indicar alteraciones del ánimo en lugar de un deterioro cognitivo real ya que los pacientes estarían hiperalertas a sus síntomas, provocando una exageración de sus déficits. 
Por otro lado, la anosognosia de los síntomas motores presente en los pacientes con EP podría influir también en estos resultados. Aunque esta no fue una variable estudiada en este estudio, se sabe que se encuentra presente en pacientes con EP con deterioro cognitivo leve ${ }^{(96)}$. Dichos pacientes presentan resultados de los test cognitivos peores a los de los controles sanos y similares a los de pacientes con EP que son conscientes de su déficit ${ }^{(97)}$ por lo que es esperable que esto también haya ocurrido en la población estudiada. En este sentido, habría que realizar más estudios que muestren la relación de la ausencia de quejas cognitivas en pacientes con EP con la presencia de anosognosia.

Al estudiar la influencia de otras patologías médicas en la CVRS, se encontró que aquellos pacientes que presentaban historia de enfermedad cardiovascular presentaban peores puntuaciones en la dimensión de movilidad y, aquellos con enfermedad pulmonar, se relacionaban a peores puntuaciones en la dimensión de actividades de la vida diaria. Estos hallazgos podrían deberse a que dichas patologías crónicas pueden provocar una mayor dificultad de movimiento y para la realización de actividades que los pacientes realizan o desean realizar. Sin embargo; no se encontró asociación de estas patologías con el PDSI.

Contrariamente a lo esperado, se encontró que los pacientes que tomaban más de un tratamiento tenían una asociación positiva con la dimensión de actividades de la vida diaria. Esto podría deberse al mayor control de los síntomas lo que les permitía realizar de forma más adecuada las diversas actividades cotidianas. Sin embargo, hay que recordar que el efecto placebo podría influir. Para obtener unas conclusiones válidas son necesarias considerar otras variables, tales como dosis equivalente de levodopa, número de comprimidos al día y scores motores y no motores.

Sobre la edad de inicio de la EP, a diferencia de otros estudios que encontraron una peor CVRS y mayor estigmatización en los pacientes con inicio temprano de enfermedad $(<50$ años), el presente estudio sólo encontró una relación negativa entre las personas que iniciaron la EP a partir de los 50 años y la dimensión de soporte social. Esto se podría explicar porque en la población estudiada, la edad media de los pacientes con inicio temprano de la EP era 10 años menor a la del resto de pacientes y; según datos de la Fundación FOESSA, la mayoría de parejas presenta una diferencia de edad $\leq 4$ años ${ }^{(98)}$ por lo que es de esperar que sus parejas sean también personas más jóvenes y saludables, con mayor capacidad de ayudarlos ${ }^{(99)}$. Sin embargo, hay que considerar también que la 
población de pacientes con EP de inicio temprano fue baja, lo que podría influir en estos resultados.

Coincidiendo con otros estudios, se encontró que el estadío de Hoehn y Yahr es un determinante significativo de la CVRS ${ }^{(45,65,100-102)}$. También se asoció al deterioro de las dimensiones de movilidad, actividades de la vida diaria, bienestar emocional y estado cognitivo. La alteración de la movilidad conllevaría en una dificultad para realizar actividades de ocio/placer lo que afectaría negativamente a la percepción de la calidad de vida del paciente ${ }^{(103,104)}$. Además, el deterioro físico afecta la realización de las actividad cotidianas, incrementan el tiempo en realizarlas o la dependencia de otras personas. Esto reduce la satisfacción personal afectando negativamente la dimensión de bienestar emocional. Por otro lado, los problemas en la concentración, memoria y el dolor corporal que se suelen presentar en etapas avanzadas de la enfermedad, impactan negativamente en la calidad de vida.

De los síntomas no motores en la EP que influyen en la CVRS, la depresión es la más estudiada siendo considerada un factor determinante del deterioro de la calidad de vida en estos pacientes. Su influencia persiste incluso en estudios con metodologías diferentes (64-66,87,93,100-101,105-107). En la primera parte del trabajo se puede observar la gran influencia de la CVRS, medida por el PDSI, sobre la depresión y; en la segunda parte, cuando se consideró a la calidad de vida como variable dependiente, la depresión también influían de forma significativa sobre la CVRS.

Además, cuando se estudió sobre qué dimensiones del PDQ-39 influía la depresión, se encontró que tenía un impacto negativo sobre la mayoría de ellas (movilidad, actividades de la vida diaria, bienestar emocional, estigmatización, estado cognitivo y comunicación). Otros estudios encontraron resultados similares ${ }^{(107-109)}$, incluido un estudio alemán que, tras agrupar las dimensiones del PDQ-39 según sus características en 3 componentes: Funcionamiento físico (dimensiones de movilidad, actividades de la vida diaria y dolor), cognición (dimensiones de comunicación y estado cognitivo) y socioemocional (bienestar emocional, estigmatización y apoyo social) ${ }^{(91)}$, también encontró influencia en dichos componentes. Esto significaría que los pacientes que presentan un mejor estado de ánimo tienen una mejor percepción de su calidad de vida, no solamente en el aspecto psicosocial, sino también en las dimensiones del funcionamiento físico. 
La ansiedad es otro síntoma no motor importante que se relaciona con la calidad de vida en la EP. En la población estudiada se encontró que, al igual que la depresión, presenta una relación bidireccional inversa con la CVRS medida por el PDSI y es un determinante significativo de la misma. Esto es consistente con estudios previos ${ }^{(78,84,86-88,101,107,110-111)}$. El mecanismo de este impacto no es claro. Es sabido que la ansiedad es más prevalente en pacientes con EP que en la población general y que en personas con otras patologías crónicas ${ }^{(112)}$. Además de la ansiedad, la personalidad ansiosa podría ser un factor de riesgo de EP en la etapa avanzada de la vida ${ }^{(34)}$. Las dimensiones que se relacionaron con la ansiedad fueron el bienestar emocional y el dolor. Una explicación sería que el deterioro en la función motora podría incrementar la sensación de dolor y, a su vez la ansiedad incrementaría estos trastornos motores, lo que ocasionaría el deterioro de la sensación de bienestar.

Los datos referidos previamente sugieren que existe una interrelación entre la calidad de vida relacionada con la salud y la presencia de ansiedad y depresión, incluso más que otras características de la enfermedad como el inicio temprano o la duración de la enfermedad. Esto es importante porque los pacientes pueden llegar a sentirse avergonzados de sus síntomas físicos, evitando situaciones sociales y aumentando la presencia de ansiedad y/o depresión ante las mismas. Esto repercutiría finalmente no solo al paciente, sino también de su entorno familiar y amical.

Este estudio presenta ciertas limitaciones como son la población estudiada, ya que todos los participantes pertenecían a una asociación de pacientes; el uso de cuestionarios autoadministrados para valorar la presencia de ansiedad o depresión, sin una evaluación completa de la salud mental de los pacientes; el haber considerado pacientes que ya estaban en tratamiento antidepresivos y ansiolítico, lo cual podría infraestimar la intensidad de los síntomas estudiados; el no haber considerado el fenotipo de la EP que podría influir en el estado de ánimo y la calidad de vida y; el no considerar la dosis equivalente de levodopa, ni la cantidad de pastillas que toman los pacientes al día. Además, no se consideró la influencia de otras variables posiblemente relacionadas, como las situaciones vitales adversas y la personalidad de los pacientes, aspectos que serían buenos a tener en cuenta en estudios futuros. 
CONCLUSIONES 


\section{CONCLUSIONES}

- Se encontró una elevada prevalencia de ansiedad y depresión en la enfermedad de Parkinson con una elevada coexistencia de ansiedad y depresión en los pacientes estudiados que podría sugerir un origen neurobiológico común de estos síntomas.

- El tiempo de evolución de la enfermedad de Parkinson influyó en la presencia de la sintomatología depresiva de la población estudiada; no así en la presencia de sintomatología ansiosa.

- La sospecha de deterioro cognitivo influyó en la presencia de sintomatología ansiosa, no así en la presencia de sintomatología depresiva.

- Se encontró una relación fuerte entre la depresión y la ansiedad y el empeoramiento de la calidad de vida relacionada con la salud.

- La presencia de un estadio más avanzado de la enfermedad de Parkinson también se relacionó a un empeoramiento de la calidad de vida relacionada con la salud; sin embargo, otras variables clínicas no mostraron relación.

- Son necesarios más estudios para ayudar a comprender la etiología de estas patologías y el impacto de las diversas intervenciones en la calidad vida de los pacientes. 
ANEXOS 
Anexo 1: Estudios de prevalencia de la enfermedad de Parkinson en España.

\begin{tabular}{|c|c|c|c|c|c|}
\hline Referencia & Diseño del estudio & Método de Screening & Criterios diagnósticos & Prevalencia Cruda & $\begin{array}{c}\text { Prevalencia por rango de } \\
\text { edad }\end{array}$ \\
\hline $\begin{array}{l}\text { Benito León et } \\
\text { al., } 2013\end{array}$ & $\begin{array}{l}\text { Transversal. } \\
\text { Puerta a puerta } \\
1994\end{array}$ & $\begin{array}{l}\text { Cuestionario y entrevista } \\
\text { seguida de evaluación por } 1 \\
\text { neurólogo }\end{array}$ & $\begin{array}{l}2 \text { de } 4 \text { criterios cardinales o } 1 \\
\text { d } 4 \text { criterios y respuesta a } \\
\text { tratamiento }\end{array}$ & $1500 / 10^{5}$ & $\begin{array}{l}65-69: 500 / 10^{5} \\
70-74: 1600 / 10^{5} \\
75-79: 1900 / 10^{5} \\
80-84: 3200 / 10^{5} \\
\geq 85: 1500 / 10^{5}\end{array}$ \\
\hline $\begin{array}{l}\text { Clavería et al., } \\
2002\end{array}$ & $\begin{array}{l}\text { Puerta a puerta } \\
1994\end{array}$ & $\begin{array}{l}\text { Cuestionario seguido de } \\
\text { evaluación por } 2 \text { neurólogos }\end{array}$ & 2 de 4 criterios cardinales & $1280 / 10^{5}$ & $\begin{array}{l}60-69: 630 / 10^{5} \\
70-79: 1300 / 10^{5} \\
80-89: 10400 / 10^{5} \\
\geq 90: 750 / 10^{5}\end{array}$ \\
\hline $\begin{array}{l}\text { Bergareche et al., } \\
2004\end{array}$ & $\begin{array}{l}\text { Puerta a puerta } \\
1996-1999\end{array}$ & $\begin{array}{l}\text { Screening por entrevistador } \\
\text { profesional; evaluación por } \\
\text { un neurólogo; evaluaciones } \\
\text { periódicas por neurólogos }\end{array}$ & $\begin{array}{l}2 \text { de } 4 \text { criterios cardinales o } 1 \\
\text { de } 4 \text { criterios y respuesta a } \\
\text { tratamiento }\end{array}$ & $1500 / 10^{5}$ & $\begin{array}{l}\text { 65-74: } 400 / 10^{5} \\
75 / 84: 4700 / 10^{5} \\
\geq 85: 2900 / 10^{5}\end{array}$ \\
\hline Errea et al., 1999 & $\begin{array}{l}\text { Transversal 1994- } \\
1996\end{array}$ & $\begin{array}{l}\text { Registros hospitales y } \\
\text { médicos seguido de } \\
\text { evaluación neurológica }\end{array}$ & UK Brain Bank & $220.6 / 10^{5}$ & $\begin{array}{l}0-39: 3.33 / 10^{5} \\
40-49: 16.5 / 10^{5} \\
50-59: 100.2 / 10^{5} \\
60-69: 435 / 10^{5} \\
70-79: 953.3 / 10^{5} \\
80-89: 973 / 10^{5} \\
\geq 90: 263.1 / 10^{5}\end{array}$ \\
\hline $\begin{array}{l}\text { Martínez Suárez } \\
\text { et al., } 2000\end{array}$ & $\begin{array}{l}\text { Fármaco- } \\
\text { epidemiológico } \\
1999 \\
\end{array}$ & $\begin{array}{l}\text { Datos de farmacia (consumo } \\
\text { de levodopa) }\end{array}$ & No utilizados & $199.1 / 10^{5}$ & No realizado \\
\hline $\begin{array}{l}\text { Criado-Álvarez } \\
\text { et al., } 1998\end{array}$ & $\begin{array}{l}\text { Fármaco- } \\
\text { epidemiológico } \\
\text { 1994-1996 }\end{array}$ & $\begin{array}{l}\text { Datos de consumo de } \\
\text { fármacos }\end{array}$ & No utilizados & $270.24 / 10^{5}$ & No realizado \\
\hline $\begin{array}{l}\text { Artazcoz Sanz et } \\
\text { al., } 1995\end{array}$ & $\begin{array}{l}\text { Fármaco- } \\
\text { epidemiológico } \\
\text { 1993-1994 }\end{array}$ & Consumo de fármacos & No utilizados & $161.5 / 10^{5}$ & No realizado \\
\hline
\end{tabular}




\section{Anexo 2: Clasificación por Estadíos de Hoehn y Yahr}

0 - No hay signos de enfermedad.

1.0 - Enfermedad exclusivamente unilateral.

1.5 - Afectación unilateral y axial.

2.0 - Afectación bilateral sin alteración del equilibrio.

2.5 - Afectación bilateral leve con recuperación en la prueba de retropulsión.

Test del empujón)

3.0 - Afectación bilateral leve a moderada; cierta inestabilidad postural, pero físicamente independiente.

4.0 - Incapacidad grave; aún capaz de caminar o de permanecer en pie sin ayuda.

5.0 - Permanece en una silla de ruedas o encamado si no tiene ayuda. 
Anexo 3: Mini-Examen Cognoscitivo (MEC-30)

Paciente

Edad.

Ocupación

Escolaridad

Fecha

...

\section{ORIENTACIÓN}

- Dígame el día fecha Estación Año

- Dígame el hospital (o lugar)

planta. ciudad Provincia Nación

\section{FIJACIÓN}

- Repita estas tres palabras ; peseta, caballo, manzana (hasta que se las aprenda)

\section{CONCENTRACIÓN Y CÁLCULO}

- Si tiene 30 ptas. y me dando de tres en tres ¿cuantas le van quedando ?

\section{MEMORIA}

- ¿Recuerda las tres palabras de antes?

\section{LENGUAJE Y CONSTRUCCIÓN}

- Mostrar un bolígrafo. ¿Qué es esto ?, repetirlo con un reloj

- Repita esta frase : ni sí, ni no, ni pero

- Coja este papel con la mano derecha dóblelo y póngalo encima de la mesa

- Lea esto y haga lo que dice : CIERRE LOS OJOS

- Escriba una frase

- Copie este dibujo

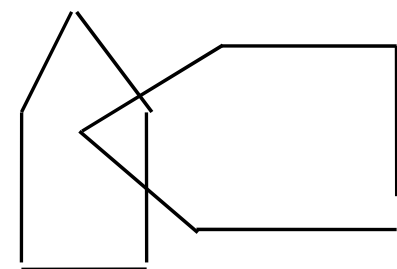

Puntuación máxima 30. 


\section{Anexo 4: CUESTIONARIO DE CALIDAD DE VIDA EN ENFERMEDAD DE PARKINSON (PDQ - 39)}

Señale con qué frecuencia ha presentado, como consecuencia de la enfermedad de Parkinson y durante el último mes, las siguientes situaciones:

\begin{tabular}{|c|c|c|c|c|c|}
\hline & Nunca & Ocasionalmente & Algunas veces & Frecuentemente & $\begin{array}{l}\text { Siempre o incapaz de } \\
\text { hacerlo (si es aplicable) }\end{array}$ \\
\hline \multicolumn{6}{|l|}{ 1. Dificultad para realizar las actividades de ocio que le gustaría hacer } \\
\hline \multicolumn{6}{|l|}{$\begin{array}{l}\text { 2. Dificultad para realizar tareas de la casa (por ejemplo, efectuar } \\
\text { reparaciones, cocinar, ordenar cosas, decorar, limpieza,...) }\end{array}$} \\
\hline \multicolumn{6}{|l|}{ 3. Dificultad para cargar con paquetes o las bolsas de la compra } \\
\hline \multicolumn{6}{|l|}{ 4. Problemas para caminar una distancia de unos 750 metros } \\
\hline \multicolumn{6}{|l|}{ 5. Problemas para caminar unos 100 metros } \\
\hline \multicolumn{6}{|l|}{$\begin{array}{l}\text { 6. Problemas para dar una vuelta alrededor de casa con tanta facilidad } \\
\text { como le gustaría }\end{array}$} \\
\hline \multicolumn{6}{|l|}{ 7. Problemas para moverse en sitios públicos } \\
\hline \multicolumn{6}{|l|}{ 8. Necesidad de que alguien le acompañara cuando salía a la calle } \\
\hline \multicolumn{6}{|l|}{ 9. Sensación de miedo o preocupación por si se caía en público } \\
\hline \multicolumn{6}{|l|}{ 10. Permanecer confinado en casa más tiempo del que usted desearía } \\
\hline \multicolumn{6}{|l|}{ 11. Dificultades para su aseo personal } \\
\hline \multicolumn{6}{|l|}{ 12. Dificultades para vestirse solo } \\
\hline \multicolumn{6}{|l|}{ 13. Problemas para abotonarse la ropa o atarse los cordones de los zapatos } \\
\hline \multicolumn{6}{|l|}{ 14. Problemas para escribir con claridad } \\
\hline \multicolumn{6}{|l|}{ 15. Dificultad para cortar los alimentos. } \\
\hline \multicolumn{6}{|l|}{$\begin{array}{l}\text { 16. Dificultades para sostener un vaso o una taza sin derramar el } \\
\text { contenido. }\end{array}$} \\
\hline \multicolumn{6}{|l|}{ 17. Sensación de depresión } \\
\hline \multicolumn{6}{|l|}{ 18. Sensación soledad y aislamiento } \\
\hline \multicolumn{6}{|l|}{ 19. Sensación de estar lloroso o con ganas de llorar } \\
\hline & Nunca & Ocasionalmente & Algunas veces & Frecuentemente & $\begin{array}{l}\text { Siempre o incapaz de } \\
\text { hacerlo (si es aplicable) }\end{array}$ \\
\hline
\end{tabular}




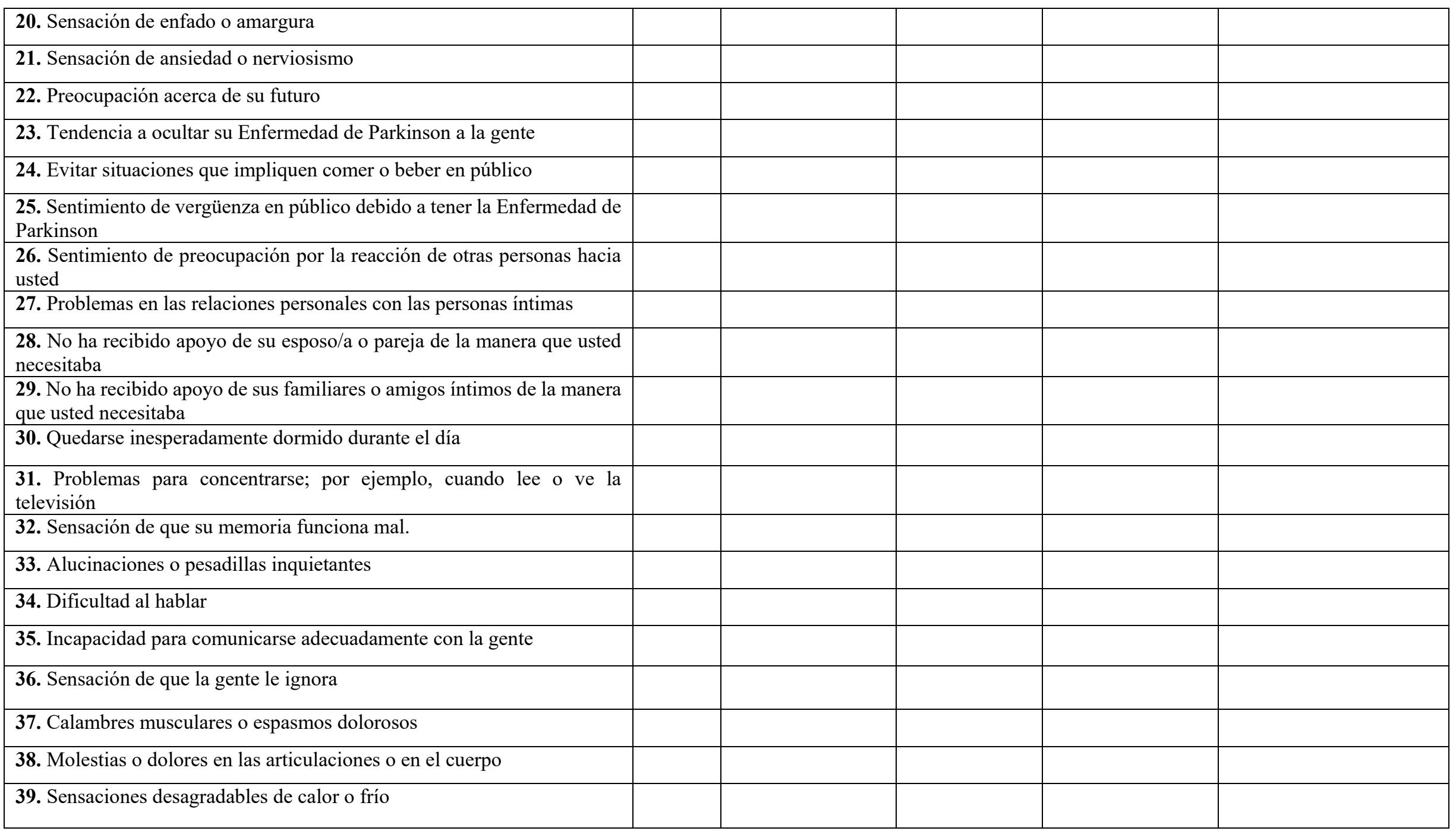




\section{Anexo 5: Cuestionario de STAI}

Instrucciones: A continuación encontrará unas frases que se utilizan corrientemente para describirse uno a sí mismo. Lea cada frase y señale la puntuación de 0 a 3 que indique mejor cómo se siente usted AHORA MISMO, en este momento. No hay respuestas buenas ni malas. No emplee demasiado tiempo en cada frase y conteste señalando la respuesta que mejor describa su situación presente.

\begin{tabular}{|c|c|c|c|c|c|}
\hline & & Nada & Algo & Bastante & Mucho \\
\hline 1. & Me siento calmado & 0 & 1 & 2 & 3 \\
\hline 2. & Me siento seguro & 0 & 1 & 2 & 3 \\
\hline 3. & Estoy tenso & 0 & 1 & 2 & 3 \\
\hline 4. & Estoy contrariado & 0 & 1 & 2 & 3 \\
\hline 5. & Me siento cómodo (estoy a gusto) & 0 & 1 & 2 & 3 \\
\hline 6. & Me siento alterado & 0 & 1 & 2 & 3 \\
\hline 7. & $\begin{array}{l}\text { Estoy preocupado ahora por posibles desgracias } \\
\text { futuras }\end{array}$ & 0 & 1 & 2 & 3 \\
\hline 8. & Me siento descansado & 0 & 1 & 2 & 3 \\
\hline 9. & Me siento angustiado & 0 & 1 & 2 & 3 \\
\hline 10. & Me siento confortable & 0 & 1 & 2 & 3 \\
\hline 11. & Tengo confianza en mí mismo & 0 & 1 & 2 & 3 \\
\hline 12. & Me siento nervioso & 0 & 1 & 2 & 3 \\
\hline 13. & Estoy desasosegado & 0 & 1 & 2 & 3 \\
\hline 14. & Me siento muy atado (como oprimido) & 0 & 1 & 2 & 3 \\
\hline 15. & Estoy relajado & 0 & 1 & 2 & 3 \\
\hline 16. & Me siento satisfecho & 0 & 1 & 2 & 3 \\
\hline 17. & Estoy preocupado & 0 & 1 & 2 & 3 \\
\hline 18. & Me siento aturdido y sobreexcitado & 0 & 1 & 2 & 3 \\
\hline 19. & Me siento alegre & 0 & 1 & 2 & 3 \\
\hline 20. & En este momento me siento bien. & 0 & 1 & 2 & 3 \\
\hline
\end{tabular}




\section{Instrucciones:}

A continuación encontrará frases que se utilizan corrientemente para describirse uno a sí mismo. Lea cada frase y señale la puntuación de 0 a 3 que indique mejor cómo se siente usted EN GENERAL, en la mayoría de las ocasiones. No hay respuestas buenas ni malas. No emplee demasiado tiempo en cada frase y conteste señalando la respuesta que mejor describa cómo se siente usted generalmente.

\begin{tabular}{|c|c|c|c|c|c|}
\hline & & Nada & Algo & Bastante & Mucho \\
\hline 1. & Me siento bien & 0 & 1 & 2 & 3 \\
\hline 2. & Me canso rápidamente & 0 & 1 & 2 & 3 \\
\hline 3. & Siento ganas de llorar & 0 & 1 & 2 & 3 \\
\hline 4. & Me gustaría ser tan feliz como otros & 0 & 1 & 2 & 3 \\
\hline 5. & Pierdo oportunidades por no decidirme pronto & 0 & 1 & 2 & 3 \\
\hline 6. & Me siento descansado & 0 & 1 & 2 & 3 \\
\hline 7. & Soy una persona tranquila, serena y sosegada & 0 & 1 & 2 & 3 \\
\hline 8. & Veo que las dificultades se amontonan y no puedo con ellas & 0 & 1 & 2 & 3 \\
\hline 9. & Me preocupo demasiado por cosas sin importancia & 0 & 1 & 2 & 3 \\
\hline 10. & Soy feliz & 0 & 1 & 2 & 3 \\
\hline 11. & Suelo tomar las cosas demasiado seriamente & 0 & 1 & 2 & 3 \\
\hline 12. & Me falta confianza en mí mismo & 0 & 1 & 2 & 3 \\
\hline 13. & Me siento seguro & 0 & 1 & 2 & 3 \\
\hline 14. & No suelo afrontar las crisis o dificultades & 0 & 1 & 2 & 3 \\
\hline 15. & Me siento triste (melancólico) & 0 & 1 & 2 & 3 \\
\hline 16. & Estoy satisfecho & 0 & 1 & 2 & 3 \\
\hline 17. & Me rondan y molestan pensamientos sin importancia & 0 & 1 & 2 & 3 \\
\hline 18. & Me afectan tanto los desengaños, que no puedo olvidarlos & 0 & 1 & 2 & 3 \\
\hline 19. & Soy una persona estable & 0 & 1 & 2 & 3 \\
\hline 20. & $\begin{array}{l}\text { Cuando pienso sobre asuntos y preocupaciones actuales, me } \\
\text { pongo tenso y agitado. }\end{array}$ & 0 & 1 & 2 & 3 \\
\hline
\end{tabular}

Compruebe si ha contestado a todas las frases con una sola respuesta. 


\section{Anexo 6: Baremos de Puntuaciones del STAI}

\begin{tabular}{|c|c|c|c|c|c|c|c|c|c|}
\hline \multirow{4}{*}{ Centiles } & \multicolumn{8}{|c|}{ Puntuaciones directas } & \multirow{4}{*}{ Decatipos } \\
\hline & \multicolumn{4}{|c|}{ Adolescentes } & \multicolumn{4}{|c|}{$\begin{array}{l}\text { Adultos } \\
\end{array}$} & \\
\hline & \multicolumn{2}{|c|}{ Varones } & \multicolumn{2}{|c|}{ Mujeres } & \multicolumn{2}{|c|}{ Varones } & \multicolumn{2}{|c|}{ Mujeres } & \\
\hline & Estado & Rasgo & Estado & Rasgo & Estado & Rasgo & Estado & Rasgo & \\
\hline 99 & $47-60$ & $46-60$ & $53-60$ & $49-60$ & $47-60$ & $46-60$ & $54-60$ & $49-60$ & 10 \\
\hline 97 & 45 & 41 & 44 & 13 & 13 & $39-40$ & 49 & 45 & 9 \\
\hline 96 & 44 & 40 & $42-43$ & 42 & 42 & 38 & 48 & 44 & 9 \\
\hline 95 & 43 & 39 & 41 & 41 & $40-41$ & 37 & 47 & 43 & 9 \\
\hline 90 & 38 & 33 & 39 & 36 & 37 & 33 & 41 & 40 & 8 \\
\hline 89 & 37 & 32 & 38 & 35 & 36 & 32 & 40 & 39 & 8 \\
\hline 85 & 36 & 30 & 36 & 33 & 33 & 29 & 37 & 37 & 8 \\
\hline 80 & 34 & 28 & 34 & 31 & 30 & 27 & 34 & 34 & 7 \\
\hline 77 & 32 & 27 & 33 & 30 & 29 & 26 & 32 & 33 & 7 \\
\hline 75 & 31 & 26 & 31 & 29 & 28 & 25 & 31 & 32 & 7 \\
\hline 70 & 28 & 24 & 28 & 27 & 25 & 24 & 29 & 30 & 7 \\
\hline 65 & 26 & 23 & 26 & 26 & 23 & 23 & 26 & 29 & 6 \\
\hline 60 & 24 & 22 & 25 & 25 & 21 & 21 & 24 & 27 & 6 \\
\hline 55 & 22 & 21 & 23 & 23 & 20 & 20 & 23 & 26 & 6 \\
\hline 50 & 20 & 20 & 22 & 22 & 19 & 19 & 21 & 24 & 6 \\
\hline 45 & 19 & 19 & 20 & 21 & 18 & 18 & 19 & 23 & 5 \\
\hline 40 & 17 & 18 & 19 & 20 & 16 & 17 & 18 & 21 & 5 \\
\hline 35 & 16 & 17 & 18 & 19 & 15 & 16 & 17 & 20 & 5 \\
\hline 30 & 14 & 16 & 17 & 18 & - & 15 & 16 & 18 & 4 \\
\hline 25 & 13 & 15 & 16 & 17 & 14 & 14. & 15 & 17 & 4 \\
\hline 23 & - & - & 15 & - & 13 & - & 14 & + & 4 \\
\hline 20 & 12 & 14 & 14 & 16 & 12 & 13 & 13 & 16 & 4 \\
\hline 15 & 11 & 13 & 13 & 15 & 10 & 11 & 12 & 14 & 3 \\
\hline 11 & - & - & 12 & 14 & 9 & 10 & 11 & 13 & 3 \\
\hline 10 & 10 & 12 & 11 & 13 & 8 & 9 & 10 & 12 & 3 \\
\hline 5 & 9 & 11 & 8 & 12 & 6 & 8 & 7 & 11 & 2 \\
\hline 4 & 8 & 10 & 7 & 11 & 5 & 7 & 6 & 10 & 2 \\
\hline 1 & $0-2$ & $0-6$ & $0-4$ & 0.7 & $0-2$ & $0-4$ & $0-2$ & $0-7$ & 1 \\
\hline $\mathrm{N}$ & 146 & 151 & 169 & 180 & 295 & 318 & 365 & 387 & $\mathrm{~N}$ \\
\hline Media & 22.35 & 21.30 & 23.28 & 23.42 & 20.54 & 20.19 & 23.30 & 24.99 & Media \\
\hline $\begin{array}{l}\text { Desv. } \\
\text { Típica }\end{array}$ & 11.03 & 8.53 & 10.56 & 9.10 & 10.56 & 8.89 & 11.93 & 10.05 & $\begin{array}{l}\text { Desv. } \\
\text { Típica }\end{array}$ \\
\hline
\end{tabular}




\section{Anexo 7: Cuestionario de Beck}

Elija de cada uno de estos 21 planteamientos, el que mejor refleje su opinión:

1. a. No me siento triste.

b. Me siento triste.

c. Me siento siempre triste y no puedo salir de mi tristeza.

d. Estoy tan triste e infeliz que no puedo soportarlo.

2. a. No me siento especialmente desanimado ante el futuro.

b. Me siento desanimado con respecto al futuro.

c. Siento que no tengo nada que esperar.

d. Siento que en el futuro no hay esperanza y que las cosas no pueden mejorar.

3. a. No creo que sea un fracaso.

b. Creo que he fracasado más que cualquier persona normal.

c. Al recordar mi vida pasada, todo lo que puedo ver es un montón de fracasos.

d. Creo que soy un fracaso absoluto como persona.

4. a. Obtengo tanta satisfacción de las cosas como la que solía obtener antes.

b. No disfruto de las cosas de la manera en que solía hacerlo.

c. Ya no tengo verdadera satisfacción de nada.

d. Estoy insatisfecho y aburrido de todo.

5. a. No me siento especialmente culpable.

b. no me siento culpable en buena parte del tiempo.

c. Me siento culpable casi siempre.

d. Me siento culpable siempre.

6. a. No creo que esté siendo castigado.

b. Creo que puedo ser castigado.

c. Espero ser castigado.

d. Creo que estoy siendo castigado.

7. a. No me siento decepcionado de mí mismo.

b. Me he decepcionado a mí mismo.

c. Estoy disgustado conmigo mismo.

d. Me odio.

8. a. No me creo ser peor que los demás.

b. Me critico por mis debilidades o errores.

c. Me culpo siempre por mis errores.

d. Me culpo.

9. a. No pienso en matarme.

b. Pienso en matarme, pero no lo haría.

c. Me gustaría matarme.

d. Me mataría si tuviera la oportunidad.

10. a. No lloro más de lo de costumbre.

b. Ahora lloro más de lo que lo solía hacer.

c. Ahora lloro todo el tiempo.

d. Solía poder llorar, pero ahora no puedo llorar aunque quiera.

11. a. Las cosas no me irritan más que de costumbre.

b. Las cosas me irritan más que de costumbre.

c. Estoy bastante irritado o enfadado una buena parte del tiempo. 


\section{d. Ahora me siento irritado todo el tiempo.}

12. a. No he perdido el interés por otras cosas.

b. Estoy menos interesado en otras personas que de costumbre.

c. He perdido casi todo el interés por otras personas.

d. He perdido todo mi interés por otras personas.

13. a. Tomo decisiones casi siempre.

b. Postergo la adopción de decisiones más que de costumbre.

c. Tengo más dificultad para tomar decisiones que antes.

d. Ya no puedo tomar decisiones.

14. a No creo que mi aspecto sea peor que de costumbre.

b. Me preocupa el hecho de parecer viejo sin atractivos.

c. Tengo que obligarme seriamente con mi aspecto, y parezco poco atractivo.

d. Creo que me veo feo.

15. a. Puedo trabajar tan bien como antes.

b. Me cuesta más esfuerzo empezar a hacer algo.

c. Tengo que obligarme seriamente para hacer cualquier cosa.

d. No puedo trabajar en absoluto.

16. a. Puedo dormir tan bien como antes.

b. No puedo dormir tan bien como solía.

c. Me despierto una o dos horas más temprano que de costumbre y me cuesta mucho volver a dormir.

d. Me despierto varias horas antes de lo que solía y no puedo volver a dormir.

17. a No me canso más que de costumbre.

b. Me canso más fácilmente que de costumbre.

c. Me canso sin hacer nada.

d. Estoy demasiado cansado como para hacer algo.

18. a. Mi apetito no es peor que de costumbre.

b. Mi apetito no es tan bueno como solía ser.

c. Mi apetito está mucho peor ahora.

d. Ya no tengo apetito.

19. a. No he perdido mucho peso, si es que he perdido algo, últimamente.

b. He rebajado más de dos kilos y medio.

c. He rebajado más de cinco kilos.

d. He rebajado más de siete kilos y medio.

20. a. No me preocupo por mi salud más que de costumbre.

b. Estoy preocupado por problemas físicos como, por ejemplo, dolores, molestias estomacales o estreñimiento.

c. Estoy preocupado por mis problemas físicos y me resulta difícil pensar en otra cosa.

d. Estoy tan preocupado por mis problemas físicos que no puedo pensar en ninguna otra cosa.

21. a. No he notado cambio alguno reciente en mi interés por el sexo.

b. Estoy menos interesado en el sexo de lo que solía estar.

c. Ahora estoy mucho menos interesado en el sexo.

d. He perdido por completo el interés en el sexo. 


\section{Anexo 8: Autorización del Comité de Ética de la Investigación del Principado de Asturias}

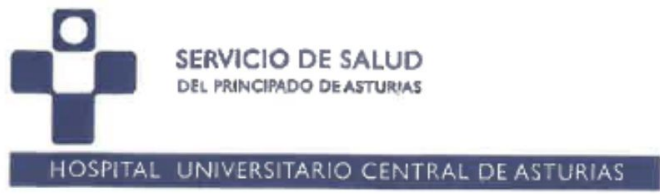

\author{
Comité de Ética de la Investigación \\ del Principado de Asturias \\ Avd. De Roma s/n \\ 33011 Oviedo \\ Tf́no: 985107927/985108028 \\ e-mail: ceicr_asturias@hca.es
}

Oviedo,26 de Marzo de 2018

Proyecto de Investigación la Investigación del Principado de Asturias, ha revisado el ANSIOSO-DEPRESIVA Investigador Principal: Dña. Fany Chuquilin CON ENFERMEDAD DE PARKINSON" de Salud Mieres Sur.

El Comité ha tomado el acuerdo de considerar que el citado proyecto reúne las condiciones éticas necesarias para poder realizarse y en consecuencia emite su
autorización. Le recuerdo que deberá guardarse la máxima confidencialidad de los datos
utilizados en este proyecto.

Fdo: Mauricio Telenti Asensio

Secretario del Comité đe Ética de la Investigación

del Principado de Asturias

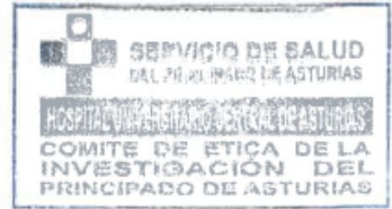


REFERENCIASS BIBLIOGRÁFICAS 


\section{Referencias Bibliográficas}

1. Olanow CW, Schapira AHV, Obeso JA. Enfermedad de Parkinson y otras discinesias. En: Kasper DL, Fauci AC, Hauser SL, Longo DL, Jameson JL, Loscalzo J, editors. Harrison. Principios de Medicina Interna. 19a ed. [Internet]. New York: McGraw-Hill Interamericana Editores; 2016 [consultado 20 enero, 2017]. p. 2609-25. Disponible en: http://harrisonmedicina.mhmedical.com/content.aspx?bookid=1717\&sectioni $\mathrm{d}=114942098$.

2. Glaría BG. Enfermedad de Parkinson y otros parkinsonismos. En: Gregorio PG, editor. Tratado de Neuropsicogeriatría. Madrid: Ergon; 2010. p. 267-94.

3. Collado-Vázquez S, Cano-de-la-Cuerda R, Carrillo JM. La enfermedad de Parkinson en la literatura, el cine y la televisión. Rev Neurol. 2014; 58 (3): 13341.

4. Fresquet-Febrer JL. James Parkinson (1755-1824). [Internet]. Historia de la medicina.org. [consultado 2 febrero, 2017]. Disponible en: http://www.historiadelamedicina.org/pdfs/parkinson.pdf

5. Goetz CG. The history of Parkinson's disease: Early descriptions and neurological therapies. Cold Spring Harb Perspect Med [Internet]. 2011 [consultado 22 enero, 2017]; 1: a008862. Disponible en: https:// doi:10.1101/cshperspect.a008862.

6. García-Ramos R., López-Valdés E, Ballesteros L, Jesús S, Mir P. Informe de la Fundación del Cerebro sobre el impacto social de la enfermedad de Parkinson en España. Neurología. 2016; 31(6):401-13.

7. Grupo de Estudios de Trastornos del Movimiento. Sociedad Española de Neurología. "Guía Oficial de Práctica Clínica en la Enfermedad de Parkinson". 2017

8. Anuario estadístico de España 2016. Demografía. [Internet] INE. Instituto Nacional de Estadística. 2016 [consultado 20 marzo, 2017]. p. 39. Disponible en: http://www.ine.es/prodyser/pubweb/anuarios_mnu.htm

9. Anuario estadístico de España 2016. Demografía. [Internet] INE. Instituto Nacional de Estadística. 2016 [consultado 20 marzo, 2017]. p. 40. Disponible en: http://www.ine.es/prodyser/pubweb/anuarios_mnu.htm 
10. Federación Española de Parkinson. [Internet]. [Consultado 01 marzo, 2017]. Disponible

en: http://www.fedesparkinson.org/index.php?r=site/page\&id=360\&title=Premio s_y_reconocimientos\&idm $=95$

11. Asociación Parkinson Asturias. [Internet]. [Consultado 3 marzo, 2017]. Disponible en: http://www.parkinsonasturias.org/la-asociacion/index.html

12. Dächsel JC, Farrer MJ. LRRK2 and Parkinson disease. Arch Neurol. 2010; 67(5):542-7.

13. Klein C, Westenberger A. Genetics of Parkinson's disease. Cold Spring Harb Perspect Med [Internet]. 2012 [consultado 22 marzo, 2017]; 2:a008888. Disponible en: https://doi: 10.1101/cshperspect.a008888.

14. Tolosa Sarró E. Enfermedad de Parkinson y otros trastornos del movimiento. En: Farreras-Rozman. Medicina Interna. [Internet]. ELSEVIER España, S.A. [consultado 3 marzo, 2017]. p. 1395-401. Disponible en: http://clinicalkeyes.asturias.csinet.es/?ACTION=CHECKED\&DATA $=\mathrm{j} 1 \mathrm{AhLNvxNPCr5O0W}$ cpj93ncTqbz1htfENHD15c8HDQBbTUxDc161gp2FwhyIWH8YO77cD95B 3rn\%2F\%0Apww8y0KXBI1\%2FZymA85ZYNSJU71t6IBDHFkN5DUGcDO V5UQFdAk\%2BfXCn5aB2fni9iZBzMWDq71Mg8\%0AQ7VHIELFKmYCCt JwLJs\%3D\%0A\#!/content/book/3-s2.0-B9788490229965001733

15. Sesar-Ignacio Á. Diagnóstico de la enfermedad de Parkinson y técnicas auxiliares. En Guía oficial de práctica clínica en la enfermedad de Parkinson 2009. Sociedad Española de Neurología. p. 53-4.

16. Cacabelos R. Parkinson's disease: From pathogenesis to pharmacogenomics. Int J Mol Sci [Internet]. 2017 [consultado 10 marzo, 2018]; 18(3):551. Disponible en: https://doi:10.3390/ijms 18030551.

17. Srivanitchapoom P, Pitakpatapee Y, Suengtaworn A. Parkinsonian síndromes: A review. Neurol India. 2018; 66:15-25.

18. Martínez-Fernández R, Gasca-Salas C, Sánchez-Ferro A, Obeso JA. Actualización en la enfermedad de Parkinson. Rev. Med. Clin. Condes. 2016; 27(3):363-79.

19. Radhakrishnan D, Goyal V. Parkinson's disease: A review. Neurol India. 2018; 66:26-35. 
20. Marsili L, Rizzo G, Colosimo C. Diagnostic criteria for Parkinson's disease: From James Parkinson to the concept of prodromal disease. Fron. Neurol. 2018; 9:156.

21. Malek N, Lawton MA, Grosset KA, Bajaj N, Barker RA, Ben-Shlomo Y, et al. Utility of the new Movement Disorder Society clinical diagnostic criteria for Parkinson's disease applied retrospectively in a large cohort study of recent onset cases. Parkinsonism Relat Disord. 2017; 40:40-6.

22. Cano-Vindel, A, Miguel-Tobal, J J Emociones negativas (ansiedad, depresión e ira) y salud. I Congreso Virtual de Psiquiatría 1 de Febrero - 15 de Marzo 2000 [consultado: 10 marzo 2017]; conferencia 28-CI-A: [29 pantallas]. Disponible en: http://www.psiquiatria.com/congreso/mesas/mesa28/conferencias/28_ci_ a.htm

23. Macía Y. Ansiedad, ira y tristeza-depresión en la enfermedad de Parkinson. [tesis doctoral]. Madrid (España): Universidad Complutense de Madrid; 2006.

24. Reijnders JSAM, Ehrt U, Weber WEJ, Aarsland D, Leentjens AFG. A systematic review of prevalence studies of depression in Parkinson's disease. Mov Disord [Internet]. 2008 [consultado 20 marzo, 2017]; 23(2):183-9. Disponible en: https://doi:10.1002/mds.21803

25. Fernández-Prieto M, Lens M, López-Real A, Puy A, Dias-Silva JJ, Sobrido MJ. Alteraciones de la esfera emocional y el control de los impulsos en la enfermedad de Parkinson. Rev Neurol. 2010; 50 (Supl 2):S41-S49.

26. Stella F, Bucken-Gobbi L, San’tAna-Simoes C. Síntomas depresivos y trastorno motor en pacientes con enfermedad de Parkinson. Rev Neurol. 2007; 45:594-8.

27. Schrag A. Quality of life and depression in Parkinson's disease. J Neurol Sci [Internet]. 2006 [consultado 20 marzo, 2017]; 248(1-2):151-7. Disponible en: https://doi:10.1016/j.jns.2006.05.030.

28. Katsarou Z, Bostantjopoulou S, Hatzizisi O, Giza E, Soler-Cardona A, Kyriazis G. ¿Factores inmunes o depresión? La fatiga relacionada con la enfermedad de Parkinson. Rev Neurol. 2007; 45(12):725-8.

29. Chaudhuri K R, Healy DG, Schapira AHV. Non-motor symptoms of Parkinson's disease: diagnosis and management. Lancet Neurol. 2006; 5:23545. 
30. Loa G, Krystkowiak P, Godefroy O. Anhenonia in Parkinson's disease: an overview. J Neuropsychiatry Clin Neurosci. 2012; 24(4):444- 51.

31. Starkstein SE, Dragovic M, Dujardin K, Marsh L, Martínez-Martín P, Pontone GM, et al. Anxiety has specific syndrome profiles in Parkinson disease: A datadriven approach. Am J Geriatr Psychiatry [Internet]. 2014 [consultado 20 marzo, 2017]; 22(12):1410-7. Disponible en: https://doi.org/10.1016/j.jagp.2013.09.006

32. Walsh K, Bennett G. Parkinson's disease and anxiety. Postgrad Med J. 2001; 77:89-93

33. Menza MA, Plaermo B, DiPaola R, Sage JI, Ricketts MH. Depression and anxiety in Parkinson's disease: posible effect of genetic variation in the serotonin transporter. J Geriatr Psychiatry Neurol. 1999; 12(2):49-52.

34. Bower JH, Grossardt BR, Maraganore DM, Ahlskog JE, Colligan RC, Geda YE, et al. Anxious personality predicts an increased risk of Parkinson's disease. Mov Disord. 2010; 25(13):2105-13.

35. Arabia G, Grossardt BR, Geda YE, Carlin JM, Bower JH, Ahlskog JE, et al. Increased risk of depressive and anxiety disorders in relatives of patients with Parkinson disease. Arch Gen Psychiatry. 2007; 64(12):1385-1392.

36. Dissanayaka NNW, White E, O’Sullivan JD, Marsh R, Pachana NA, Byrne GJ. The clinical spectrum of anxiety in Parkinson's disease. Mov Disord. 2014; 29(8):967-74.

37. Dissanayaka, NNW, Sellbach A, Matheson S, O’Sullivan JD, Silburn PA, Byrne GJ, \& et al. Anxiety disorders in Parkinson's disease: prevalence and risk factors. Mov Disord [Internet]. 2010 [consultado 20 marzo, 2017]; 25(7):838-845. Disponible en: https://doi: 10.1002/mds.22833.

38. Grupo OMS sobre la calidad de vida. ¿Qué calidad de vida? Foro Mundial de la Salud. The World Health Organization Quality of Life Assessment (WHOQOL). 1996; 17:385-7

39. Fernández-López JA, Fernández-Fidalgo MF, Cieza A. Los conceptos de calidad de vida, salud y bienestar analizados desde la perspectiva de la clasificación internacional del funcionamiento (CIF). Rev Esp Salud Pública. 2010; 84:169-84

40. CIF. Clasificación internacional del funcionamiento, de la discapacidad y de la salud. [Internet]. Organización Mundial de la Salud. 2001. [consultado 20 
marzo,

http://www.imserso.es/InterPresent2/groups/imserso/documents/binario/435ci f.pdf

41. Velarde-Jurado E, Ávila-Figueroa C. Evaluación de la calidad de vida. Salud Púbica Mex. 2002; 44: 349-61.

42. Frades-Payo B, Forjaz MJ, Martínez-Martín P. Situación actual del conocimiento sobre calidad de vida en la enfermedad de Parkinson: II. Determinantes y factores asociados. Rev Neurol. 2009; 49: 655-60.

43. Navarro-Peternella FM, Marcon SS. Calidad de vida de las personas con enfermedad de Parkinson y su relación con la evolución en el tiempo y la gravedad de la enfermedad. Rev. Latino-Am. Enfermagem. 2012; 20(2): [08 pantallas].

44. Arango GP, Bolaños AM., Fernández BE. Calidad de vida y discapacidad en personas con enfermedad de Parkinson de la ciudad de Cali 2011. [tesis]. Cali (Colombia): Universidad Autónoma de Manizales. Grupo de Investigación Cuerpo-Movimiento; 2012

45. Hanna KK, Cronin-Golomb AC. Impact of anxiety on quality of life in Parkinson's disease. Parkinsons Dis [Internet]. 2012 [consultado 20 marzo, 2017]; 640707. Disponible en: https://doi:10.1155/2012/640707.

46. Martínez-Martín P, Jeukens-Visser M, Lyons KE, Rodríguez-Blazquez C, Selai C, Siderowf A, et al. Health-related Quality-of-life scales in Parkinson's disease: Critique and recommendations. Mov Disord. 2011; 26(13):2371-2380.

47. Frades-Payo B, Forjaz MJ, Martínez-Martín P. Situación actual del conocimiento sobre calidad de vida en la enfermedad de Parkinson: I. Instrumentos, estudios comparativos y tratamientos. Rev Neurol. 2009; 49: 594-8.

48. Martinez-Martin P, Schapira AH, Stocchi F, Sethi, K, Odin P, MacPhee G, et al. Prevalence of nonmotor symptoms in Parkinson's disease in an international setting; study using nonmotor symptoms questionnaire in 545 patients. Mov. Disord. 2007; 22(11):1623-9.

49. Inoue T, Kitagawa M, Tanaka T, Nakagawa S, Koyama T. Depression and major depressive disorder in patients with Pakinson's disease. Mov Disord. $2010 ; 25(1): 44-9$ 
50. Broen MPG, Narayen NE, Kuijf ML, Dissanayaka NNW, Leentjens AFG. Prevalence of anxiety in Parkinson's disease: A systematic review and metaanalysis. Mov Disord. 2016; 00(00):1-9.

51. Spielberg CD, Gorsuch RL, Lushene RE. Cuestionario de Ansiedad EstadoRasgo. Manual. SATI. 4ta Ed. Madrid, España.: TEA Ediciones, 1994.

52. Livia O, Manrique E. Niveles de ansiedad, cogniciones dentales negativas y capacidad de control en la atención odontológica. Rev Psiquiatr Salud Ment. 2001; 2(1-2):0-0.

53. González-Arranz, B, Saúl LA. Evaluación del cambio del contenido de los constructos personales de una muestra de personas con depresión que ha pasado por un proceso psicoterapéutico. Revista de Psicoterapia. 2017; 28 (107):295-316.

54. Fernández Cuervo EM. Niveles de ansiedad en los pacientes oncológicos del área maxilofacial y su relación con las manifestaciones signosintomatológicas de la articulación temporo-mandibular [tesis doctoral]. Oviedo (España): Universidad de Oviedo; 2016.

55. Beato MS, Pulido RF, Pinho MS, Gozalo M. Reconocimiento falso y ansiedad estado/rasgo. Psicológica. 2013; 34: 299-311.

56. Herrero H, Caserío S, Morante MAA, Montero M, De La Cruz J, Pallás CR. Estudio de los niveles de ansiedad en los profesionales de una unidad neonatal. An Pediatr (Barc). 2012; 77(1):22-7.

57. De Molina-Fernández I, Rubio-Rico L, Roca-Biosca A, Jimenez-Herrera M, De la Flor-López M, Sirgo A. Ansiedad y miedos de las gestantes ante el parto: la importancia de su detección. Revista Portuguesa de Enfermagem de Saúde Mental [Internet]. 2015 [consultado 20 marzo, 2017]; 13(23):17-24. Disponible en: http://www.scielo.mec.pt/pdf/rpesm/n13/n13a03.pdf.

58. López Sánchez M. Evaluación neurocognitivo-emocional en la enfermedad de Pelizaeus-Merzbacher (PMD). Rev Dis Cli Neuro. 2018; 5(1):15-30.

59. Rodríguez Puente Z. Estado emocional y gestación: La influencia del nivel de ansiedad materno en los resultados perinatales [tesis de máster en internet]. León (España): Universidad de León, 2014. [consultado 20 marzo, 2017]. Disponible en: https://buleria.unileon.es/bitstream/handle/10612/5149/2015.\%20Zaida\%20R odr $\%$ C3\%ADguez $\% 20$ Puente.pdf? sequence $=1$ 
60. Arreaza A, Correnti M, Battista B. Ansiedad como rasgo de la personalidad de un grupo de pacientes con liquen plano bucal. Acta odontol. Venez. 2010; 48(2):1-9.

61. Ryu YJ, Chun EM, Lee JH, Chang JH. Prevalence of depression and anxiety in outpatients with chronic airway lung disease. Korean J Intern Med, 2010; 25(1):51-7.

62. Girón RM, Fernandes G, Cisneros C, Gómez-Punter M, Segrelles G, Ancochea J. Trastornos psicológicos y calidad de vida en pacientes con bronquiectasias no relacionadas con fibrosis quística. Arch Bronconeumol. 2013; 49(10):41520.

63. Consejo General de Colegios Oficiales de Psicólogos. Evaluación del inventario BDI-II. [Internet]. Madrid (España). 2013 [consultado 20 marzo, 2017]. Disponible en: https://www.cop.es/uploads/PDF/2013/BDI-II.pdf.

64. Nicoletti A, Mostile G, Stocchi F, Abbruzzese G, Ceravolo R, Cortelli P, et al. Factors influencing psychological well-being in patients with Parkinson's disease. PLoS ONE [Internet]. 2017 [consultado 10 marzo, 2018]; 12(12):e0189682. Disponible en: https://doi.org/10.1371/journal. pone.0189682.

65. Wiesli D, Meyer A, Fuhr P, Gschwandtner U. Influence of Mild Cognitive Impairment, Depression, and Anxiety on the Quality of Life of Patients with Parkinson Disease. Dement Geriatr Cogn Disord, Extra [Internet]. 2017. [consultado 12 febrero, 2018]; 7:297-308. Disponible en: https://doi.org/10.1159/000478849

66. Rosińczuk J, Kołtuniuk A. The influence of depression, level of functioning in everyday life, and illness acceptance on quality of life in patients with Parkinson's disease: a preliminary study. Neuropsychiatric Disease and Treatment. 2017; 13:881-7.

67. Williams JR. The Declaration of Helsinki and public health. [Internet]. WHO. 2008 [consultado 22 marzo, 2017]; 86:650-1. Disponible en: https://www.wma.net/es/20activities/10ethics/10helsinki/index.html19.

68. Real Decreto 561/1993, de 16 de abril, por el que se establecen los requisitos para la realización de ensayos clínicos con medicamentos. BOE n.॰114, de 13 mayo de 1993, p. 14346-64. 
69. Dissanayaka NNW, Sellbach A, Silburn PA, O’Sullivan JD, Marsh R, Mellick GD. Factors associated with depression in Parkinson's disease. J Affect Disord [Internet]. 2011 [consultado 22 marzo, 2017]; 132:82-88. Disponible en: https://doi:10.1016/j.jad.2011.01.021.

70. Nuti A, Ceravolo R, Piccini A, Dell'Agnello G, Bellini G, Gambaccini G, et al. Psychiatric comorbidity in a population of Parkinson's disease patients. Eur J Neurol. 2004; 11:315-20.

71. Yamanishi T, Tachibana H, Oguru M, Matsui K, Toda K, Okuda B, et al. Anxiety and Depression in Patientes with Parkinson's disease. Intern Med. 2013; 52:539-45.

72. Álvarez-Avellón $\mathrm{T}$, Arias-Carrión $\mathrm{O}$, Menéndez-González $\mathrm{M}$. Neuropsychiatric symptoms and associated caregiver stress in geriatric patients with Parkinson's disease. J Neurol Neurosci. 2015; 1(5):1-8.

73. Aguilar M. Depression (BDI-II) in Parkinson's disease: prevalence, types, and variables associated to major, minor and subclinical depression in COPPADIS cohort. Comunicación presentada en el $22^{T H}$ International Congress of Parkinson's Disease and Movement Disorders. 2018; Hong Kong, China.

74. Nêgre-Pagês L, Grandjean H, Lapeyre-Mestre M, Montastruc JL, Fourrier A, Lépine JP, et al. Anxious and depressive symptoms in Parkinson's disease: The French Cross-Sectionnal DoPaMiP Study. Mov Disord. 2010; 25(2):157-66.

75. Cui SS, Du JJ, Fu R, Lin YQ, Huang P, He YC, et al. Prevalence and risk factors for depression and anxiety in Chinese patients with Parkinson disease. BMC Geriatr [Internet]. 2017 [consultado 22 febrero, 2018]; 17:270. Disponible en: https://doi.org/10.1186/s12877-017-0666-2.

76. Perrin AJ, Nosova E, Co K, Book A, Iu O, Silva V, et al. Parkinsonism Relat Disord [Internet]. 2017 [consultado 23 marzo 2018]; 36:93-7. Disponible en: https://doi: 10.1016/j.parkreldis.2016.12.02

77. Errea JM, Ara JR. Depresión y enfermedad de Parkinson. Rev Neurol. 1999; 28(7):694-8.

78. Kovács M, Makkos A, Weintraut R, Karádi K, Janszky J, Kóvacs N. Prevalence of anxiety among Hungarian subjects with Parkinson's disease. Behav Neurol [Internet]. 2017 [consultado 25 enero 2018]: 1470149. Disponible en: https://doi.org/10.1155/2017/1470149. 
79. Broen MPG, Leentjens AFG, Hinkle JT, Moonen AJH, Kuijf ML, Fischer NM, et al. Clinical Markers of anxiety subtypes in Parkinson disease. $J$ Geriatr Psychiatry Neurol. 2018; 31(2):55-62.

80. Leentjens AFG, Moonen AJH, Dujardin K, Marsh L, Martínez-Martín P, Richard IH, et al. Modeling depression in Parkinson disease. Disease-specific and nonspecific risk factors. Neurology. 2013; 81:1036-43.

81. Tandberg E, Larsen J, Aarsland D, Laake K, Cummings J. Risk Factors for depression in Parkinson disease. Arch Neurol. 1997; 54(5):625-30.

82. Wichowicz HM, Slawek J, Derejko M, Cubala WJ. Factors associated with depression in Parinson's disease: a cross-sectional study in a Polish population. Eur Psychiatry. 2006; 21(8):516-20.

83. Chagas MHN, Moriyama TS, Felício AC, Sosa AL, Bressan R, Ferri CP. Depression increases in patients with Parkinson's disease according to the increasing severity of the cognitive impairment. Arq Neuropsiquiatr [Internet]. 2014 [consultado 2 abril 2017]; 73(6):426-9. Disponible en: doi: 10.1590/0004-282X20140049.

84. Broen MPJ, Köhler S, Moonen AJH, Kuijf ML, Dujardin K, Marsh L, et al. Modeling anxiety In Parkinson's disease. Mov Disord. 2016; 31(3): 310-6.

85. Dissanayaka NNW, O’Sullivan JD, Pachana NA, Marsh R, Silburn PA, White EX, et al. Disease-specific anxiety symptomatology in Parkinson's disease. Int Psychogeriatr. 2016; 28(7):1153-63.

86. Opara JA, Brola W, Blaszczyk B. Quality of life in Parkinson's disease. J Med Life. 2012; 5(4):375-81.

87. Hu T, Ou R, Liu H, Hou Y, Wei Q, Song W, et al. Gender and onset age relateddifferences of non-motor symptoms and quality of life in drug-naïve Parkinson's disease. Clin Neurol Neurosurg. 2018; 175:124-9.

88. Heller J, Shulz JB, Reetz K. Evidence for gender differences in cognition, emotion and quality of life in Parkinson's disease? Aging Dis. 2014; 5(1):6375.

89. Georgiev D, Hamberg K, Hariz M, Forsgren L, Hariz GM. Gender differences in Parkinson's disease: a clinical perspective. Acta Neurol Scand. 2017; 136:570-84.

90. Lubomski M, Louise Rushworth R, Lee W, Bertram KL, Williams DR. Sex 
differences in Parkinson's disease. J Clin Neurosci. 2014; 21:1503-6.

91. Ophey A, Eggers C, Dano R, Timmermann L, Kalbe E. Health-related quality of life subdomains in patients with Parkinson's disease: the role of gender. Parkinsons dis. [Internet]. 2018 [consultado 30 Octubre 2018]: 6532320. Disponible en: https://doi.org/10.1155/2018/6532320.

92. Moore O, Kreitler S, Ehrenfeld M, Giladi N. Quality of life and gender identity in Parkinson's disease. J Neural Transm. 2005; 112:1511-22.

93. Cubo E, Rojo A, Ramos S, Quintana S, Gonzalez M, Kompoliti, K, Aguilar M. The importance of educational and psychological factors in Parkinson's disease quality of life. Eur J Neurol. 2002; 9:589-93.

94. Treves TA, Parmet Y, Klimovitzky S, Korczyn AD. The effect of schooling on reported age of onset of cognitive decline: a collaborative study. $J$ Clin Neurosci. 2016; 34:86-8.

95. Jones JD, Hass C, Mangal P, Lafo J, Oun MS, Bowers D. The cognition and emotional well-being indices of the Parkinson's disease questionnaire-39: What they do really measure? Parkinsonism Relat Disord, 2014; 20(11):123641.

96. Orfei MD, Assogna F, Pellicano C, Pontieri FE, Caltagirone C, Pierantozzi M, et al. Anosognosia for cognitive and behavioral symptoms in Parkinson's disease with mild dementia and mild cognitive impairment: Frequency and neuropsychological/neuropsychiatric correlates. Parkinsonism Relat Disord. $2018 ; 54: 62-7$.

97. Castro PCF, Aquino CC, Felício AC, Doná F, Medeiros LMI, Silva MCA, et al. Presence or absence of cognitive complaints in Parkinson's disease: mood disorder or anosognosia? Arq Neuropsiquiatr. 2016; 74(6):439-44.

98. Castro T, Seiz M. Instituto de Economía, Geografía y Demografía, CSIC. La transformación de la familia en España desde una perspectiva sociodemográfica. En: VII Informe sobre exclusión y desarrollo social en España 2014 [Internet]. Fundación FOESSA. Fomento de Estudios Sociales y de Sociología Aplicada. 2014 [consultado 10 diciembre 2019]. p. 27. Disponible en: https://www.foessa2014.es/informe/uploaded/documentos trabajo/131120140 45006 7884.pdf

99. Mehanna R, Janovic J. Young-onset Parkinson's disease: Its unique features 
and their impact on quality of life. Parkinsonism Relat Disord. 2019; 65:39-48.

100. Berganzo K, Tijero B, González-Eizaguirre A, Somme J, Lezcano E, Gabilondo I, et al. Síntomas no motores y motores en la enfermedad de Parkinson y su relación con la calidad de vida y los distintos subgrupos clínicos. Neurologia. 2017; 31(9):585-91.

101. Pandey S, Bajjaj BK, Wadhwa A, Anand KS. Impact of sleep quality on the quality of life of patients with Parkinson's disease: a questionnaire based study. Clin Neurol Neurosurg. 2016; 148:29-34.

102. Menon B, Nayar R, Kumar S, Cherkil S, Venkatachalam A, Surendran K, Deepak KS. Parkinson's disease, depression, and quality-of-life. Indian J Psychol Med. 2015; 37(2):144-7.

103. Moreira RC, Bueno M, Serra AP, Israel VL, Teive HAG (2017). Quality of life in Parkinson's disease patients: progression markers of mild to moderate stages. Arq Neuropsiquiatr [Internet]. 2017 [consultado 20 marzo 2018]; 75(8):497502. Disponible en: https://doi.org/10.1590/0004-282X20170091.

104. Mandira ALS. Study of positive influence on quality of life patients with Parkinson's disease participants Group Lotus. Rev Kairós. 2012; 15(4): 18599.

105. Santos-García, De Deus-Fonticoba T, Suárez-Castro E, Borrué C, Mata M, Solano-Vila B, et al. Non-motor symptoms burden, mood, and gait problems are rhe most significant factors contributing to a poor quality of life in nondemented Parkinson's disease patients: Results from the COPPADIS Study Cohort. Parkinsonism Relat Disord. 2019; 66:151-7.

106. Santos-García D, De la Fuente-Fernández R. Impact of non-motor symptoms on health-related and perceived quality of life in Parkinson's disease. J Neurol Sci. 2013; 332:136-40.

107. Quelhas R, Costa M. Anxiety, depression, and quality of life in Parkinson's disease. J Neuropsychiatry Clin Neurosci. 2009; 21(4):413-9.

108. Tu X-J, Hwang W-J, Ma H-I, Chang L-H, Hsu S-P. (2017). Determinants of generic and specific health-related quality of life in patients with Parkinson's disease. PLoS ONE. [Internet]. 2017 [consultado 20 marzo 2018]; 12(6): e0178896. Disponible en: https://doi.org/10.1371/journal.pone.0178896.

109. Simpson J, Lekwuwa G, Crawford T. Predictors of quality of life in people with Parkinson's disease: evidence for both domain specific and general 
relationships. Disabil Rehabil. 2014; 36(23):1964-70.

110. Balestrino R, Martinez-Martin P. Reprint of "Neuropsychiatric symptoms, behavioural disorders, and quality of life in Parkinson's disease". J Neurol Sci. 2017; 374:3-8.

111. D'lorio A, Vitale C, Piscopo F, Baiano C, Falanga AP, Longo K, et al. Impact of anxiety, apathy and reducen functional autonomy on perceived quality of life in Parkinson's disease. Parkinsonism Relat Disord. 2017; 43:114-7.

112. Pincus JH, Tucker GJ. Behavioral Neurology. 4th Ed. New York, NY: Oxford University Press; 2013. 